\title{
DEVELOPMENT FROM WITHIN: AN IN-SERVICE PUBLIC TRANSIT NARRATIVE
}

\author{
by \\ Ryan Grenon \\ A thesis submitted to \\ the Faculty of Graduate and Postdoctoral Affairs \\ in partial fulfillment of \\ the requirements for the degree of \\ MASTER OF ARTS \\ Human-Computer Interaction \\ at \\ CARLETON UNIVERSITY \\ Ottawa, Ontario \\ September, 2021
}

(C) Copyright by Ryan Grenon, 2021 


\begin{abstract}
We identify some of the underlying processes that support decision-making activities undertaken by three public transit stakeholders in Ottawa, and evaluate perceived usability of the proposed technological component of a novel public transit decisionmaking information system: a database query and results visualization tool (i.e., a cybercartographic atlas prototype). We highlight significant vulnerabilities in existing public transit decision-making processes, including the presence of common biases and heuristics, wherever human judgment is exercised. Our prototype is designed to handle the collection, organization, and visualization of public sentiment (i.e., Twitter data), and [theoretically] uses the Nunaliit Cybercartographic Atlas Framework as the underlying database. In remote usability-testing sessions, we diagnose two system-wide user interface issues that require immediate design solutions and report a mean system usability score of 90.4 with five participants. We discuss the notions of choice architecture and nudging as important design considerations that can improve precision in predictive judgments.
\end{abstract}




\section{Acknowledgements}

Thank you to Professor Robert Biddle for taking a chance on me - for allowing me the opportunity to enrol in two graduate-level courses while not formally admitted to the program, and for your guidance and support throughout this process. Your kindness truly knows no bounds.

Thank you to Professor Fraser Taylor - your mentorship and exceptional communication skills have enabled me to grow as a person and young professional. Your commitment to treating people the right way, and emphasis on building and maintaining relationships, is something that I will keep with me moving forward. You embody humility in light of dynamism, thank you.

Thank you to my therapist Doug - you allowed me the freedom to work through some difficult times at my own pace, and introduced me to your former colleague Daniel Kahneman's body of work. You listened for understanding and helped me find perspective in life with thoughtful reading suggestions and an uncanny ability to label my thoughts.

Finally, to my loving family and friends: Your unwavering love and support continues to be instrumental in any individual successes I achieve. Without your love and support none of this would have been possible. Thank you so very much. 


\section{Table of Contents}

Abstract $\quad$ ii

Acknowledgements $\quad$ iii

Chapter 1 Introduction 1

1.1 Motivation .......................... 1

1.2 Vision and Philosophy . . . . . . . . . . . . . . 4

1.3 Research Questions . . . . . . . . . . . . . . . . 7

1.4 Contributions . . . . . . . . . . . . . . . . . 7

1.5 Project Outline . . . . . . . . . . . . . . . . . 9

$\begin{array}{lll}\text { Chapter } 2 & \text { Background } & 11\end{array}$

2.1 Introduction . . . . . . . . . . . . . . . . 11

2.1.1 The Evolution of Cybercartography . . . . . . . . . . . 13

2.1.2 Cybercartography Redefined . . . . . . . . . . . . 15

2.2 The Theory and Practice of Cybercartography . . . . . . . . . . 16

2.2.1 Why Cybercartography . . . . . . . . . . . 16

2.2.2 Cybercartography and Geographic Information Systems . . . . 16

2.3 Cybercartography: A Multimodal Approach . . . . . . . . . . . . 17

2.4 Cybercartography and Volunteered Geographic Information . . . . . . 19

2.4.1 Synthesis of VGI and Cybercartography . . . . . . . . . . . 20

2.4 .2 Legal Issues with VGI . . . . . . . . . . . . . . . . . 21

2.4.3 Intellectual Property, Privacy, and Civil Liability . . . . . . 22

2.5 Cybercartography for Education . . . . . . . . . . . . . 24

2.5.1 Cybercartography and Howard Gardner's Multiple Intelligence Theory . . . . . . . . . . . . . . . . 25

2.6 The Nunaliit Cybercartographic Atlas Framework . . . . . . . . . . 28

2.6.1 The Evolution of Nunaliit . . . . . . . . . . . . . . . 30

2.6.2 Recent Developments . . . . . . . . . . . . . 33

2.6.3 Future Development of Nunaliit . . . . . . . . . . . . . 37 
$\begin{array}{lll}\text { Chapter } 3 & \text { Methodology } & 39\end{array}$

3.1 Timeline . . . . . . . . . . . . . . . . . . . . . . 39

3.1.1 Navigating the Covid-19 Global Pandemic . . . . . . . . . . . 39

3.2 Phase 1: Field Observations . . . . . . . . . . . . . . . . . . 40

3.2 .1 Town Halls . . . . . . . . . . . . . . . . . . . . . . . . . . . . 40

3.2.2 City Council and Transit Commission Meetings . . . . . . . . 41

3.2.3 Major Transit Hub Site Visits . . . . . . . . . . . . . . . . . 41

3.2.4 Social Media Observations . . . . . . . . . . . . . . . . . . 42

3.3 Phase 2: Participant Identification, Recruitment and the Semi-Structured

Interview . . . . . . . . . . . . . . . . . . . . . . . . 42

3.3.1 Participant Identification and Defining Stakeholder Groups . . 42

3.3.2 Participant Recruitment . . . . . . . . . . . . . . . 43

3.3.3 The Semi-Structured Interview . . . . . . . . . . . . . . . . 44

3.3.4 Establishing Stakeholder Mandates . . . . . . . . . . . . . . . 44

3.4 Phase 3: Prototype Build and the Remote Usability-Testing Session . 45

3.4.1 The Prototype Build . . . . . . . . . . . . . 45

3.4.2 The Remote Usability-Testing Session . . . . . . . . . . . . 56

$\begin{array}{lll}\text { Chapter } 4 & \text { Results } & 63\end{array}$

4.1 Field Observations . . . . . . . . . . . . . . . . . . 63

4.2 Semi-Structured Interviews . . . . . . . . . . . . . . . 68

4.2.1 General Findings for Stakeholder Group 1 . . . . . . . . . . . 69

4.2.2 General Findings for Stakeholder Group 2 . . . . . . . . . . . 75

4.2.3 General Findings for Stakeholder Group 3 . . . . . . . . . . . 78

4.2 .4 Summary ..................... . . . 81

4.3 Remote Usability-Testing Sessions . . . . . . . . . . . . . . . . . 82

4.3.1 General Findings for Stakeholder Group 1 . . . . . . . . . . 82

4.3.2 General Findings for Stakeholder Group 2 . . . . . . . . . . . 90

4.3.3 General Findings for Stakeholder Group 3 . . . . . . . . . . . 96

4.3.4 Post-Test Questionnaire (i.e., System Usability Scale) . . . . . 99

4.3.5 Challenges ..................... 102 
5.1 A Conceptual Framework for the Collection, Organization, and Visualization of Public Sentiment for Three Public Transit Stakeholder Groups . . . . . . . . . . . . . . . . . 108

5.2 Controlling the Master Switch . . . . . . . . . . . . . . . . . . 112

5.3 Cybercartography and the Application of Choice Architecture and Behavioural Insights to Improve Decision-Making . . . . . . . . . . . . . 114

$\begin{array}{lll}\text { Chapter } 6 & \text { Conclusion } & 119\end{array}$

6.1 General Findings . . . . . . . . . . . . . . . . . . . . . . 119

6.2 Lessons Learned . . . . . . . . . . . . . . . . . . . . . . . 121

6.3 Limitations and Future Considerations . . . . . . . . . . . . . . . . . 121

$\begin{array}{ll}\text { References } & 122\end{array}$

$\begin{array}{ll}\text { Appendices } & 131\end{array}$

Appendix A Transit Commission 132

Appendix B Cybercartography: A Multimodal Approach 136

B.0.1 Speech and Cartographic Visualization . . . . . . . . . . . 136

B.0.2 Gesture and Cartographic Visualization . . . . . . . . . . . . . 137

B.0.3 Sound and Cartographic Visualization . . . . . . . . . . . . 138

B.0.4 Haptic and Cartographic Visualization . . . . . . . . . . . . . 140

Appendix C The Inuit Siku (Sea Ice) Atlas 142

Appendix D The Arctic Bay Atlas $\quad 144$

$\begin{array}{lll}\text { Appendix E Semi-Structured Interview } & 146\end{array}$

E.1 Letter of Invitation . . . . . . . . . . . . . . . . . . 146

E.2 Screening Questionnaire . . . . . . . . . . . . . . . . . 148

E.3 Informed Consent Form . . . . . . . . . . . . . . . . . . . . 149

E.4 Semi-Structured Interview Guide . . . . . . . . . . . . . . . 152 
Appendix F Remote Usability-Testing Session 162

F.1 Letter of Invitation . . . . . . . . . . . . . . . . . . . . . . 162

F.2 Informed Consent Form _. . . . . . . . . . . . . . . . . 164

F.3 Usability-Testing Script . . . . . . . . . . . . . . . . 167

Appendix G Bus Stop Accessibility - A Mathematical Process 173 


\section{List of Tables}

4.1

SUS Results . . . . . . . . . . . . . . . . . . 101 


\section{List of Figures}

3.1 Advanced Google Search Template . . . . . . . . . . . . . 46

3.2 Empty Database Query . . . . . . . . . . . . 47

3.3 Prototyping Query Flows . . . . . . . . . . . . 48

3.4 Prototyping Result Flows . . . . . . . . . . . . . . . 49

$4.1 \quad$ Landing Page . . . . . . . . . . . . . . . 84

4.2 Stakeholder Group 1 Query . . . . . . . . . . . 86

$4.3 \quad$ Stakeholder Group 1 Results $+\ldots \ldots$. . . . . . . . 88

$4.4 \quad$ Stakeholder Group 2 Query . . . . . . . . . . . . . . 92

4.5 Stakeholder Group 2 Query Results+ . . . . . . . . . . . . . . 94

$4.6 \quad$ Stakeholder Group 3 Query . . . . . . . . . . . . . 97

$4.7 \quad$ Stakeholder Group 3 Results $\ldots \ldots$. . . . . . . . . 98

5.1 Two Decompositions of MSE . . . . . . . . . . 107

$5.2 \quad$ Four Unique Bus Stop Dialogues . . . . . . . . . . . . 115

G.1 Distribution of the Transit Commission's Bus Stop Accessibility Forecasts for One Neighbourhood . . . . . . . . . . . . . . . 174

G.2 Distribution of Errors in the Transit Commission's Bus Stop Accessibility Forecasts for One Neighbourhood . . . . . . . . . 175

G.3 Shots Fired . . . . . . . . . . . . . . . . . 175

G.4 Distribution of Errors with Bias Reduced by Half vs. Noise Reduced by Half . . . . . . . . . . . . . . . . . . . . 177 


\section{Chapter 1}

\section{Introduction}

\subsection{Motivation}

In this thesis, we document the existing public transit decision-making Information System (IS) in Ottawa, Canada, and propose a novel IS predicated on the practice of Cybercartography. We describe a methodology for the collection, organization, and visualization of public sentiment (i.e., Twitter data) for three public transit stakeholders, and discuss notions of nudging through choice architecture as a mechanism to improve decision-making outcomes. Our motivation for this project stems from a strong desire to improve the status quo for public transit decision-making in Ottawa. We believe public transit decision-making should be a community-driven exercise, best realized with a development from within approach. We describe our work in three phases:

\section{Phase 1: Field Observations}

2. Phase 2: Participant Identification, Recruitment, and the Semi-Structured Interview

3. Phase 3: Prototype Build, and the Remote Usability-Testing Session

In anticipation of the Field Observation Phase, we made a determination about what represents the public transit decision-making body, and we began the process of identifying formal and informal inputs into the public transit decision-making process. We identified the Transit Commission as the formal municipal body responsible for "ensuring the development of a safe, efficient, accessible and client-focused transit system, and for providing overall guidance and direction to the Transportation Services Department on all issues relating to the operation of public transit, including conventional bus service, the O-Train and Para Transpo". A complete list of matters 
for which the Transit Commission is responsible to City Council, and a complete list of responsibilities the Transit Commission has final decision-making authority over, is included in Appendix A. Of particular importance to the work described in this thesis, the Transit Commission has final decision-making authority over the following responsibilities [28]:

1. Determine and meet the transit needs of residents and visitors;

2. Provide excellent customer service and foster a reputation of safety and reliability; and,

3. Provide a transit service that is accessible and affordable.

A development from within approach identifies the community being served as the entity from which the development should originate. For the purpose of this thesis, we define 'community' as a collective of individuals with varying ideals, ways of perceiving and experiencing the world, who share the common interest of using public transit infrastructure in their day-to-day activities. An immeasurable amount of well-defined community consultation methodologies exist. We hope this work builds on that body of knowledge.

In his 2011 book, Thinking, Fast and Slow, the psychologist, Daniel Kahneman, offers the following insight:

"Democracy is inevitably messy, in part because the availability and the affect heuristics that guide citizens' beliefs and attitudes are inevitably biased, even if they generally point in the right direction. Psychology should inform the design of risk policies that combine the experts' knowledge with the public's emotions and intuitions" [57].

We hope to improve municipal policymaking through the implementation of a novel IS that merges community sentiment with expert knowledge, for public transit decisionmakers.

In November 2018, Ottawa mayor Jim Watson settled a legal Twitter dispute with three of his critics by unblocking them and acknowledging that his is a public 
account [36]. The three plaintiffs - lawyer and community activist Emilie Taman, Dylan Penner of the Council of Canadians and James Hutt of the Canadian Union of Postal Workers - alleged in an October 2018 lawsuit that Watson had infringed their constitutional right to freedom of expression by blocking them from his official Twitter account. They argued that his Twitter feed was a public account used in the course of his duties as mayor. As a condition of the legal settlement, Watson thanked the litigants for bringing the issue forward and encouraged all councillors to maintain "a high level of accessibility through social media". Moreover, Watson agreed to unblock all residents who had been denied access to his Twitter feed; however, he retained the ability to mute his critics online - meaning Twitter users can see his feed, but Watson will not be notified of anything they tweet to him [36]. Some may consider the mayor's behaviour anti-democratic; in light of the mayor's decision, we sought to develop a mechanism that could promote democratic process by eliminating an elected officials' ability to mute citizen participation in municipal policy discussions taking place on social media. We introduce this mechanism as the core technological component of a novel public transit decision-making IS, with the ability to collect social media data via an Application Programming Interface (API), organize and store the data in a relational-document database, and render information visualizations in an interactive cybercartographic atlas and adjacent information timeline.

For the purpose of this thesis, we define an information system as a formal, sociotechnical, organizational system, designed to collect, process, store, and distribute information [88]. From a sociotechnical perspective, information systems generally include four components: task, people, structure (or roles), and technology [81]. Our proposed IS can therefore be defined as: an integration of components for the collection, storage and processing of data, in which the data is organized by people to produce information, and contribute knowledge, including digital products, such as a cybercartographic atlas. We note the 'Nunaliit Cybercartographic Atlas Framework', described in chapter 2 , as the core technological component of the proposed IS.

As we conducted field observations, we noted various community inputs into the public transit decision-making process. We identified Town Halls, and City Council and Transit Commission meetings as formal organized inputs into the public transit 
decision-making process, due to the opportunity for public delegation in the presence of elected, and or appointed municipal officials [28, 29]. Subsequent to our initial observations, we made a concerted effort to explore unorganized and informal inputs into the public transit decision-making process. We observed the Ottawa community using major transit hubs during morning and afternoon peak-service hours, and we passively observed the public transit conversation happening on the social media platform, Twitter, with the intent to observer public transit discussion that may not present in more formal venues, e.g., at Ottawa City Hall.

Our field observations highlighted several opportunities to grow citizen participation in public transit decision-making. We identified 'Persons [living] With Disabilities' (PWD), who often experience additional barriers when using public transit infrastructure, as a growing community that is currently underserved in terms of access to public transit infrastructure and access to public transit decision-making. Accordingly, we began to conceptualize an interactive tool that could, in theory, strengthen the collective voice of the PWD community in public transit decision-making, thereby increasing democratic process. We believe public transit infrastructure that is readily accessible to the PWD community is an issue of importance to society, and we believe that increased community access to public transit decision-making serves to benefit all citizens, an idea described in greater detail in chapter 5 .

Public transit planning in western societies is considered a top-down process, headed by municipal governments [124]. Town Halls, committee meetings, community engagement surveys, and other forms of implied public consultation are characterized as inputs into public transit decision-making; however, these activities often amount to tokenism, creating an illusion of democratic process. We intend to challenge the public-transit decision-making status quo by leveraging the social capital ingrained in community narratives, visualized in cybercartographic atlas', realised by public transit stakeholders - including public transit decision-makers.

\subsection{Vision and Philosophy}

The vision and philosophies that underpin the ideas presented in this thesis must be attributed to many insightful thought leaders. Distinguished Research Professor 
Fraser Taylor, pioneered development in the theory and practice of Cybercartography, as is described in the Modern Cartography Series Developments in the Theory and Practice of Cybercartography [21]. We introduce several key elements in the theory and practice of Cybercartography in chapter 2. In chapter 5, we introduce a conceptual framework for the collection, organization, and visualization of public sentiment for three public transit stakeholder groups in Ottawa.

The practice of Cybercartography and the innovative Nunaliit Cybercartographic Atlas Framework has been used to produce cybercartographic atlases in several countries, spanning multiple continents [110]. Taylor and his research team have received over 14 million dollars in research funding from a variety of sources including: SSHRC, CFI, CEC-NAPECA, INAC, the Ontario Research Fund, the Ontario Brain Institute, and the Government of Nunavut, etc. [21]. In multiple applications, Cybercartography has been applied to work what economists refer to as the 'local knowledge problem': the observation that the data required for rational economic planning are distributed among individual actors and thus unavoidably exist outside the knowledge of a central authority [51]. We observe and engage with the community, and design and evaluate a prototype, fit with a mock cybercartographic atlas, with three public transit stakeholders, in an attempt to collect and organize the data required for public transit planning activities.

In chapter 5, we propose a conceptual framework, and introduce the notion of a 'master switch': a body or person in an IS, who maintains the ability to extend the community narrative to the intended audience, or not. The term 'master switch' can be traced to Fred W. Friendly and the advent of public-access television cable TV channels - "At stake is not the First Amendment or the right of free speech, but exclusive custody of the master switch" [127]. The term has also, more recently, been attributed to Tim Wu, following the success of his 2010 book The Master Switch: The Rise and Fall of Information Empires [127]. In this thesis, we highlight the importance of the community constructing its own authentic narrative, while also maintaining control over the narrative throughout the IS. As we describe in chapter 4 , the existing public transit decision-making IS involves public sentiment moving through traditional media organizations and managing editors, who may decide to 
report on public transit issues, or not, and in a manner that is, or is not, consistent with the community's perspective. The social capital inherent in cybercartographic products is correlated with their ability to show many perspectives of complex societal problems and their solutions, to multiple stakeholders with varying mandates, in an dynamic and interactive environment [110].

In a historical context, the practice of Cybercartography is discussed as a knowledge generating exercise in which a community produces and consumes knowledge throughout the iterative process of cybercartographic product design [110]. When we consider the objective of improving policymaking - a second variable should be considered. When we design information visualizations for public transit stakeholders, the goal is to actually improve decision-making outcomes, and not merely educate stakeholders about community sentiment, although educated stakeholders is a niceto-have. In order to improve decision-making outcomes, we consulted thought leaders at the intersection of policy, law and technology.

The psychologists Daniel Kahneman and Amos Tversky pioneered work that challenged the rational model of judgement and decision-making, while clinical psychologist Paul E. Meehl provided foundational arguments for the use of simple, statistical rules in place of intuitive clinical judgments, i.e., algorithms in favour of expert judgement [57, 73]. Legal scholar Cass R. Sunstein and economist Richard H. Thaler worked on many of the core ideas that underpin Nudge theory. They argued that people are quite susceptible to counterproductive behavioural and cognitive tendencies, therefore, it is appropriate for social planners and policymakers to modify decision-making situations in ways that nudge people toward better choices [114]. They distinguish 'nudging' from 'forcing' by the fact that people can still choose the de-emphasized option relatively easily. In our conceptual framework, we apply the concepts of 'nudging' and 'choice architecture', but from the perspective of the community - i.e., the community narrative should be framed using choice architecture to nudge decision-makers toward better decision-making outcomes, realized through effective policymaking. Choice architecture should mitigate the impact of heuristics and biases, such as anchoring effects, the illusion of understanding and validity and 
the law of small numbers, and ultimately regress decision-making to the mean - opting for policymaking that combines the community's emotions and intuitions with expert knowledge.

\subsection{Research Questions}

Taylor defines Cybercartography as "a complex holistic concept which is in simplest terms the application of location-based technologies to the analysis and understanding of issues of importance to society, and the dissemination of the results in ways that people can easily understand through cybercartographic atlases" [110]. Cybercartography has been influenced by international development theory, and Taylor has long argued that development is best realized from a bottom-up perspective, which emphasizes the prioritization of local community knowledge [103, 112]. Building on international development theory and the theory and practice of Cybercartography, we address two core research questions in this thesis:

1. What are the needs of the PWD community in terms of access to public transit infrastructure, and access to the public transit decision-making process? What barriers to access exist, and what can be done to reduce the number of barriers?

2. How must the existing public transit decision-making IS change in order to incentivize community narratives over intuitive judgements, at the policymaking level?

\subsection{Contributions}

In this thesis, we identify some of the underlying processes that support decisionmaking activities undertaken by three public transit stakeholders, and evaluate perceived usability of the proposed technological component of a novel public transit decision-making IS: a database query and results visualization tool (i.e., a mock cybercartographic atlas prototype). Our work highlights significant vulnerabilities in existing public transit decision-making processes, including the presence of common biases and heuristics, wherever human judgment is exercised. Our prototype is designed to handle the collection, organization and visualization of public sentiment for 
three public transit stakeholders in Ottawa. The principal contributions of this work are:

1. In the course of conducting field observations and a semi-structured interview, we identity some of the underlying processes that support decision-making activities undertaken by three public transit stakeholders in Ottawa, and highlight significant vulnerabilities in decision-making processes that occur wherever human judgment is exercised.

2. We build on the community consultation literature by extending the definition of a municipal working group. At the municipal government level, a 'working group' is considered a small cohort of individuals, typically 4-8 persons, who are consulted at regular intervals by city planners, in order to provide insight about the needs of a community. We propose an application of Cybercartography, in which the municipal working group is redefined as the public transit advocate/lobby community, who use the database query and results visualization tool to facilitate the collection and organization of public sentiment for public transit decision-makers, such that multiple independent representations on the same item can be visualized and considered together. When several independent representations on the same item are visualized and considered together, the resulting judgment (i.e., averaged judgment) is generally considered less noisy (the notions of noise and bias in human judgment are introduced in chapter 5).

3. We design and evaluate a low-fidelity prototype, fit with a mock cybercartographic atlas and adjacent information timeline, with three public transit stakeholders in Ottawa. In a remote usability-testing session, we evaluate two use cases using a database query and results exploration task, with three participant groups. We diagnose two system-wide usability issues, and report on perceived system usability. We note feedback related to functional and non-functional requirements for any potential software build. Finally, we discuss the notions of choice architecture and nudging as important design considerations that can improve precision in predictive judgments. 


\subsection{Project Outline}

The work described in this thesis was completed over a period of twenty-four months. The work is described three phases:

\section{Field Observations:}

Field observations were imperative in defining the public transit stakeholders discussed in this project. The practice of Cybercartography gives control and perspective to the community, and recognizes the importance of a diversity of opinion, rather than black and white solutions [110]. Accordingly, we sought to observe the public transit conversation from multiple perspectives. We passively participated in Town Halls, City Council and Transit Commission meetings, visited major transit hubs during peak-service hours, and passively observed the public transit conversation happening on the social media platform, Twitter. We noted the perspectives of the bureaucrat, the subject expert, the private sector, the non-government advocate/lobby, and the PWD community who rely on accessible public transit infrastructure in their day-to-day activities. The traditional map rarely reflects multiple perspectives, while the cybermap allows for the presentation of multiple views, thus revealing some of the complexities of the challenges and potential responses to complex societal problems, such as public transit planning [110]. Our field observations served to define three stakeholders involved public transit decision-making in Ottawa.

2. Participant Identification, Recruitment, and the Semi-Structured Interview:

We recruited participants via direct message using social media accounts, and through public web profiles that included personal contact information (e.g., email address, phone number, etc.). Contact information for prospective participants was collected throughout the field observation phase, and stored in a secure location until we received CUREB-B clearance to begin participant recruitment. Our initial recruitment goal was three participants for each of the three stakeholder groups we identified - for a total of nine participants; 
however, we managed to successfully recruit six participants to take part in the study during the Covid-19 Global Pandemic. All participants took part in one thirty-minute semi-structured interview and one forty-five-minute remote usability-testing session. The purpose of the semi-structured interview was to learn about issues of importance to the PWD community, directly related to public transit infrastructure and access to the public transit decision-making process. We curated three versions of the semi-structured interview - one for each public transit stakeholder group. During the interview, all participants were asked to describe their recent experience using the public transit system, and to describe their use of the social media platform, Twitter, to participate in the public transit conversation happening on the platform. The semi-structured interview served to define the existing public transit decision-making IS (i.e., how does public sentiment travel from the citizen to the Transit Commission, and what is the role of the public transit advocate/lobby in the existing IS).

3. Prototype Build, and the Remote Usability-Testing Session:

From the semi-structured interview, we compiled lists of functional and nonfunctional requirements to be weighed and considered as part of the prototyping process. In short, core functional requirements included the provision of three unique user profiles, one for each stakeholder group, each with its own set of data permissions (e.g., add, delete, share, etc.). Core non-functional requirements included accessibility, learnability, and legal and licensing considerations (described in detail in chapter 2). A cybercartographic atlas is, by definition, dynamic and interactive, and often presents multiple information representations on a complex subject. In contrast, a prototype is, by definition, a first, typical or preliminary model of something, and is not dynamic or interactive beyond the feature (s) being evaluated.

The use of royal we in this thesis is intended to refer to ' $\mathrm{I}$ ' in consultation with my supervisor. It is an acknowledgement that the work described therein is a product of a community of knowledge. Consistent with the iterative development of a cybercartographic product, a master's thesis is not completed in isolation from others. 


\section{Chapter 2}

\section{Background}

\section{$2.1 \quad$ Introduction}

Cybercartography was formally introduced as a term in the keynote address at the 1997 International Cartographic Association Conference in Stockholm, Sweden, by Fraser Taylor [107]. The term represented the culmination of over two decades of thinking about the evolution of cartographic theory and practice; Bill Cartwright summarized Taylor's work [23]:

In 1991 Taylor proposed a model that more appropriately described the true profile of contemporary mapping [105]. He developed the model to contribute to what he described as the 'New Map'. Taylor (1994) further developed his model to expand the 'communication' aspects, from just visual display techniques to include both the visual and non-visual [106]. The revised model included 'interaction' and 'dynamics' that led to improved communications and analysis as well as a new range of cartographic products. Later, Taylor (1997, 2003) expanded the model to introduce the use of the Web as a method of communicating new media cartographic artefacts and the concept of 'Cybercartography' was born [10\%, 10\%].

The initial definition of Cybercartography was heavily influenced by practice, as opposed to theory. The importance of the main artefact of Cybercartography, the cybercartographic atlas, remains relevant today, but recent thinking and applications characterize the importance of the process by which an atlas is produced as equally, if not more important, than the resulting product itself [110]. In 2003, Taylor defined Cybercartography as "the organization, presentation, analysis, and communication of spatially referenced information on a wide variety of topics of interest and use to society in an interactive, dynamic, multimedia, multisensory, and multidisciplinary format" [108]. Taylor identified six key ideas about Cybercartography, which should 
be considered as an extension of the formal definition, to emphasize the holistic and iterative interaction between the theory and practice, which differentiate Cybercartography form other mapping approaches. The six key ideas of Cybercartography are:

1. Individuals use all of their senses while observing what is around them: $\mathrm{Cy}-$ bercartography is therefore exploring the possibilities of using all five senses in its representations, in order to make cybercartographic atlases as reflective as possible of human sensory realities;

2. Individuals have different learning preferences, and prefer teaching and learning in different formats and environments; Cybercartographic atlases have great potential in both formal and informal education, as they provide the same information in multiple formats, allowing users the freedom to choose which format or combination of formats and modalities they wish to use.

3. Educational theory suggests that individuals learn best when they are actively, rather than passively, involved. This applies both in formal and informal learning situations. Engaging the user requires carefully thought out interactive engagement strategies, including the design of the user interfaces;

4. The social media revolution has given people the power to create their own narrative, and cartography is challenged to respond to individual and community needs in ways that previously did not exist. The Nunaliit Cybercartographic Atlas Framework is a software platform that provides a mechanism for people to enter their own data into cybercartographic atlases. Cybercartography provides a means for people to tell their own stories, as part of a holistic information package. The Nunaliit Cybercartographic Atlas Framework is an open source platform, which provides a meta-data structure for the input of data or information, and was designed with an interface that does not require special knowledge in order to input information;

5. Many topics of interest to society are complex, and the same set of 'facts' on 
these topics, for example, climate change, are open to a variety of representations. Even when there may be agreement on the facts, there can often be a wide variety of interpretations. There are often no simple 'right' or 'wrong' answer to many complex questions. Cybercartography allows the presentation of different ontologies or narratives on the same topic, without privileging one over another. The user can consider the various narratives presented, and have a greater understanding of the complexities and uncertainties surrounding many topics. Traditionally, the map was an authoritative source. Cybermaps are much more nuanced; and,

6. Traditional cartography is a supply-driven industry. National mapping agencies supplied the definitive and authoritative maps for public consumption. Technological advances have led to a more demand-driven approach, and with it, a focus on individuals and communities creating their own maps, and making their own choices about what to represent and what not to represent in any given map. Individuals are now 'prosumers' rather than 'consumers' of mapping products, and as a result, are democratizing mapping in new ways. Indigenous communities, for example, have often been largely 'invisible' on state produced maps, or have been represented by others outside their community. The practice of $\mathrm{Cy}-$ bercartography gives voice to indigenous communities and other marginalized community groups, both literally and metaphorically $[109,110]$.

In summary, a cybercartographic atlas is a metaphor for the analysis of all kinds of qualitative and quantitative information of interest to society, organized temporalspatially, and displayed in innovative multi-sensory and multimedia formats, which people readily understand [110]. Cybercartographic atlases allow communities to tell their own stories; both mapping and storytelling are basic human instincts, and are a central part of the holistic nature of Cybercartography.

\subsubsection{The Evolution of Cybercartography}

The practice of Cybercartography requires a transdisciplinary approach. Two of the early cybercartographic atlases, "The Atlas of the Antarctica" and "The Atlas of 
Canada's Trade with the World", were constructed by teams spanning twelve different academic disciplines, in conjunction with community and government partners [110]. Unlike many conventional maps, cybercartographic atlases cannot be created by an individual with expertise in a single area. The mock cybercartographic atlas described in this thesis is no different. The primary researcher was formally trained in the social sciences, with graduate specialization in human-computer interaction techniques and user-centred design philosophy. Education, experience, extensive field observations and community consultation was necessary to learn about the community's values, and how these values might be represented in a cybercartographic atlas. An understanding of quantitative and qualitative social research approaches was necessary to conduct semi-structured interviews with three public transit stakeholder groups in Ottawa, and a working knowledge of human-computer interaction techniques and user-centred design philosophy guided the prototype design process.

The presentation of multiple perspectives and solutions to complex problems requires dynamic user interaction techniques and tiered access to information, based on different stakeholder needs. The interaction techniques and user permissions that guide user interactions in cybercartographic atlases permit the conveyance of relevant information and knowledge for atlas users with different information needs. This differs from the normative, accurate nature of Geographic Information Systems (GIS), which are often viewed as definitive and authoritative mediums, and produced with a single user profile in mind. Cybercartography has a strong qualitative element and often includes art, theatre, and a range of human activities, emotions and sentiments that have not been traditionally mapped [110]. We address the importance of multi-modal and multisensory information visualizations, in the context of cybercartographic atlas' in chapter 3. The practice of Cybercartography represents a shift in control of the mapping process in terms of what is mapped and who controls these decisions $[37,72]$.

The traditional distinction between 'map producer' and 'map user' has dissolved, and co-production of knowledge is now the norm, not the exception [110]. Perspectives are not confined to the end user's interactions with information visualized in an atlas; they also apply to data relations (i.e., relational-document structures), data 
presentations and media format, and include formats not currently mediated by geographical information systems. For example, the Atlas of Arctic Bay includes a rap video produced by local youth titled Don't call me Eskimo, whose lyrics serve as a revelation of the many social and economic problems facing youth in Canada's Northern communities [110].

\subsubsection{Cybercartography Redefined}

The formal definition of Cybercartography has matured significantly since its initial introduction as a term in 1997 [107, 109, 110]. In 2005, Taylor amended the formal definition of Cybercartography to include a description of the seven major elements of Cybercartography, and in 2014, Taylor reclassified those seven major elements of Cybercartography into the aforementioned six central ideas that define Cybercartography as a concept and practice [111].

Most recently (2019), Taylor defined Cybercartography as "a complex, holistic user-centred process which applies location-based technologies to the analysis of topics of interest to society, and the presentation of the results in innovative ways through cybercartographic atlases" [110]. This refined definition reflects changes in understanding that arose from applications in new areas of practice, such as Indigenous mapping in international contexts and new forms of linguistic cartography and language mapping in Mexico and other international destinations [110]. Recent applications of Cybercartography, as a form of language mapping, has furthered discussion of Cybercartography as an inherently collaborative research process that situates communities of map users at the centre of atlas production, while incorporating the complementary nature of cartographic data using interactive, multimodal information visualizations. This is can be summed up in the plurality of all facets of knowledge that arise from the prosumption of cybercartographic atlases: plurality of sources of information, contributors, perspectives, methods, scales, goals and modalities for communication and interaction [4]. 


\subsection{The Theory and Practice of Cybercartography}

\subsubsection{Why Cybercartography}

In 1997, Taylor argued that we must move away from narrow, technological, normative and formalistic approaches to cartography, and proceed in search of a more holistic approach, where both mapping as a process and the map as a product are expanded [107]. Much of Taylor's early work embodied his commitment to reassert and demonstrate the importance and utility of maps and mapping in the information era [110]. Map-making fulfils one of our deepest human desires: understanding the world around us, and our place in it. Hence, a map-maker's willingness to venture beyond the boundaries of geography or conventions of historical representation would not require a map if they had complete understanding of the world and their place in it. The dominant paradigm in recent years for cartographers has been - cartography as a science [71]. While Cybercartography does employ a strong scientific component, the presence of qualitative elements ultimately serve to increase map utility without compromising the structure and order infused by quantitative elements [110].

\subsubsection{Cybercartography and Geographic Information Systems}

There are significant differences between Cybercartography and both Geographic Information Systems (GIS) and geographic information science. Martinez and Reyes (2006) argue that Cybercartography owes much to the concept of cybernetics and second-wave sociocybernetics, and in-particular, that general systems theory and modelling and complexity theory constitute the building blocks of modern cybercartographic atlases, which are primarily social products [91]. Whereas, GIS was initially conceived as a form analytical cartography, a subdiscipline of cartography and the spatial data sciences, and defined in terms of its development [76, 77, 117]. Taylor (2019) argues that GIS and GIScience are more in line with Norbert Weiner's concept of a scientifically objective first-order cybernetic system, where the observer is not viewed as part of the map-making process [110]. Further differentiating Cybercartography from GIS and GIScience, Taylor (2019) highlights the aspect of map co-production 
(i.e., cybercartographer + community), a process that takes place within the communities the atlas' are designed to serve, and describes the process as interactive, in both the technological and sociological sense. The process of Cybercartography is qualitatively different from simply consulting the intended users in order to determine their needs, which many GIS systems do. In the practice of Cybercartography, the map user plays an integral role in the determination of design priorities, is involved throughout the iterative design process, and is an active user of the end product [110]. Reyes and Martinez also view researchers engaged in the process of Cybercartography as participants in an active social process, and not merely as 'objective' and 'scientific' observers [91].

\subsection{Cybercartography: A Multimodal Approach}

Traditional hard copy (i.e., paper) maps are not interactive and do not permit user optimization (i.e., user-centred design) of the visual field to the same degree as computer generated maps. Traditional hard copy maps were often marked up with a writing utensil, or folded to direct the user's attention to a specific area, or highlighted with translucent markers, or examined in greater detail with the use of a magnifying glass [71]. Technological advances have led to the adoption of new computer generated interactive mapping systems, with enhanced interaction tools that build on early forms of user interaction with non-computer generated maps. Computer generated dynamic mapping systems, for example, online GIS maps and invehicle route-navigation assistance devices, allow a user to browse, sort, and perceive information in a manner that aligns with their specific goals, i.e., a user-centred design $[1,126,118]$.

Cartography is primarily a visual discipline. A major design goal in the production of hard copy maps is to reduce visual search time for the user [87]. Modern computer generated mapping systems challenge the early design limitations of hard copy maps, by reducing visual search time through the creation of active workspaces, which permit the user to manipulate the map by scrolling and zooming the display [10]. Additional visual display variables such as blur, focus and transparency were introduced to expand user customization of the map display and contribute to a 
more user-centred experience. Blur can give symbols a fuzzy appearance and is used to visualize uncertainty, while focus is used to highlight a specific area and attract the user's attention. Both transparency and shading, or shadowing, can be used to simulate a three-dimensional look [62]. Visual variables commonly deployed in computer generated maps act to manipulate the user's visual field, a practice that allows the map designer to guide a user's attention, while maintaining the overall context of the map information [32, 69]. Interactive maps further reduce visual search time by switching from a unimodal visual approach, to a multimodal approach that employs combinations of sensory modalities, such as visual, auditory and touch to direct the user's attention [55]. The practice of Cybercartography builds on the primarily visual discipline of cartography through the implementation of user interactions that make use of all human sensory modalities [110].

In a general sense, a multimodal interface uses multiple modalities to convey information and promotes stronger bidirectional communication between the user and the mapping system [110]. A multimodal interface should consider how different modalities can be combined to produce natural user interactions that promote a state of flow, without triggering information overload [110]. The design of a multimodal interface should promote efficient and natural interactions between the user and the mapping system [84, 43]. Moreover, a multimodal interface should be deployed to help direct a user's attention to the most critical piece of information within a mapping system, at a specific moment in time. Multimodal interfaces empower the user to perform a wide range of resource intensive activities, as the cognitive workload can be spread across multiple modalities. Traditional hard copy maps require a user's singular attention, as information is communicated primarily through the visual modality [52].

Cybercartographic visualization is a term that denotes the formation of mental models of temporal-spatial information (e.g., the Great Lakes System, at a specific moment in time), which would otherwise be large to observe [110]. Individuals use maps to build mental models of temporal-spatial relationships among data (e.g., the movement of a weather pattern or trade patterns, but also, the movement of people across land). The usefulness of the visualizations can be greatly enhanced when the information is communicated using multiple sensory modalities. The bidirectional, 
multimodal conveyance of information through cybercartographic atlases facilitates communication between the map user and the mapping system [110]. Bertin (1983) formalized a visual variable syntax that cartographers often employ when designing maps. His variable syntax considered: geographic position in the plane, size, value, texture, colour, orientation, and shape, and he also proposed a set of rules to govern the use of the variable syntax, which considered whether the data was quantitative, nominal, or ordinal [11]. Subsequent researchers have supplemented Bertin's original syntax with variables such as hue, saturation, crispness, resolution, and transparency [71]. The evolution of visual mapping variables helped to establish a common base syntax that promotes consistent usage of map symbols among cartographers and map users around the world.

Appendix B includes four additional sections that address the potential contributions of four non-visual modalities to cartographic visualization. The modalities are: speech, gesture, sound, and haptic (i.e., touch). The relative advantages and limits, and the cognitive workload implications for each modality are discussed.

\subsection{Cybercartography and Volunteered Geographic Information}

The increasingly location-based nature of public discourse and information sharing by cartographic experts, geographic specialists and neogeographers is well into its second decade $[67,120]$. The result is a shift in the paradigm of web cartography. In recent years, we have noted a rise of user-centred design, including the design of user interfaces, dynamic map content and mapping functions, and the democratization of map-making led by the general public and amateur cartographers [118]. The shift toward a cartographic web represents a general broadening of the nature of geographic information collection, including distribution and use, from an activity traditionally dominated by a relatively small community of expert users to one that embraces diverse communities of geographic information 'prosumers' (a portmanteau of producer and consumer) [20,49, 110].

The collaborative and assertive approach of communities toward geographic information has prompted numerous fundamental changes to the ways in which maps are produced and consumed, with cartographic interactivity (e.g., the customization, 
population, and dissemination of digital maps online through the actions of multiple participants) being among the most significant of the new paradigm shifts [94]. In this paradigm, the 'cartographic web' represents a natural spatial extension of the prevailing archetype of social media and the Web 2.0 model [104].

In the new era of cartographic interactivity, new forms of geographic information are generated directly and indirectly as manifestations of individuals' daily routines, such as commuting in a GPS equipped vehicle, or capturing multimedia content with location information enabled [44]. The geographic information is used to populate collaborative road atlases, to aid in reporting and monitoring, and in relief coordination of natural disasters (e.g., Hurricane Harvey), to coordinate and respond to riots, protests, and other politically motivated gatherings, to augment existing repositories of location-based multimedia, and to share information among hobbyist communities $[44,68,61,38,2]$.

On a global scale, Volunteered Geographic Information (VGI) is regularly contributed to location-based services that enhance, and may one day replace, public and private sector web-based products $[46,128]$. Although still in its infancy, the theory

and practice of VGI is in many ways parallel to that of Cybercartography [110]. The subsequent section presents a critical analysis of Cybercartography and its potential as a unifying framework for VGI. Opportunities and challenges in both theory and practice, and consideration of the current legal aspects of VGI and its relationship to what has been termed 'authoritative' information are discussed.

\subsubsection{Synthesis of VGI and Cybercartography}

Cybercartography represents a powerful tool for the management, dissemination, and visualization of geographic narratives, or geo-narratives, derived from heterogeneously sourced VGI [110]. It offers an unprecedented opportunity for rethinking the way location-based information is produced, collected, engaged with, disseminated, and represented. Cybercartography emphasizes the relationships and capabilities engendered between developers, facilitators, and community members in the production of a cybercartographic atlas, and it describes entirely new processes and results in new products of engaged cartographic interactivity [110]. 
While location is a central organizing principle in the practice of Cybercartography, cybercartographic atlases are also capable of ingesting, cataloguing, and representing non-location-based information [110]. The Nunaliit Cybercartographic Atlas Framework uses an unstructured schema-less document database system that promotes optimal information management, scalability and performance. The cybercartographic approach represents a new way of thinking about VGI management that is scalable, flexible, and responsive to unforeseen needs and uses that may arise as an atlas matures. Each atlas can respond to the changing and expanding needs of the community by adapting to new and unanticipated forms of qualitative or qualitative data, new unexpected relationships between types of information, and new categories of users, contributors and consumers of the atlas, who for security or other reasons may be limited to some subset of the entire collection contained within an atlas [110].

Dissemination of an atlas is managed at the level of the community, and is based on a community's needs for information sharing and or protection [110]. The Nunaliit Cybercartographic Atlas Framework is designed specifically to handle multiple layers of information that can be coded to user types, such that information presented in an atlas is determined by the status of the user [110]. In many cases, this means a simple dichotomy between a public and a private layer; however, more complex systems may be developed as needed by the community.

Geo-narratives, oral histories, life histories and biographies told not only in time but also in space, are at the heart of the cybercartographic approach [110]. The Nunaliit Cybercartographic Atlas Framework, although rooted in the ontological paradigm of point, (poly)line and polygon common to GIS software, does not prescribe in advance the visual representation of the VGI contributed by the community. Hence, symbology, palette, and other aesthetic choices are left to the community, and then incorporated by researchers and developers [110].

\subsubsection{Legal Issues with VGI}

The incorporation of VGI in cybercartographic products provides an opportunity for expansion in terms of the content that may be incorporated into an atlas, and it also redefines the nature of individual and community participation in a project [110]. 
The inclusion of VGI can raise a number of legal issues that are less likely to present in traditional top-down mapping projects, or that manifest differently in more conventional mapping contexts, such as GIS [110]. The traditional, top-down, highly controlled development process of 'official' and often state sponsored cartographic products has rendered legal considerations largely invisible, such that the conventions of state driven cartography have evolved to ensure that the creation and dissemination processes of cartographic products fit within the parameters of local government laws [110]. In the context of cybercartographic products and VGI, it is necessary to consider the impact of legal rules on the design and use of cybercartographic products. Issues such as intellectual property rights, privacy, ethics, and civil liability should be considered in the production of cybercartographic products that include VGI [110].

\subsubsection{Intellectual Property, Privacy, and Civil Liability}

In copyright language, a cybercartographic atlas is considered a multimedia work; put differently, it is a compilation consisting of several different works. While the overall author or authors of the compilation will have copyright in the atlas itself, where VGI is relied upon, it is likely that multiple individual contributors will have uploaded their own photos, video, or test-based content to the compilation. The result is that there is likely to be a large number of works embedded in the compilation, each of which may have its own copyright, resulting in a large number of different rights holders [98]. Therefore, the creators of a cybercartographic atlas that incorporates VGI should consider how rights among the different contributions will be managed. There are several strategies to address copyright issues in cybercartographic products. One strategy is to require contributors to assign copyright in their works to the creators of the cybercartographic product, but this may serve as a deterrent to potential contributions, as well as counterproductive to the open and collaborative goals of the process of Cybercartography [110]. A less invasive strategy is to require contributors to give the project a non-exclusive license to reproduce and disseminate the contributed works. If the cybercartographic product in question does not have a foreseeable termination point, a perpetual license is likely preferable [110].

It may be the case that some of the contributed content will be works to which 
the contributor does not hold the rights, and is thus not in a position to license them to the creators of the atlas. For example, an individual may upload a photograph taken by a friend (or retrieved online), or they may contribute text, audio or video content for which they are not the author. This can leave the atlas creators vulnerable to accusations of copyright infringement [110]. In many cases, even if complaints are received about specific contributions to an atlas, it may be enough for the atlas builder to remove the offending content promptly. This may or may not be an issue for the creators of the atlas, depending upon how unique the contribution is, how important that information is to the goals of the atlas, or whether there are concerns that the complaints are motivated by a desire to suppress the telling of particular stories via the cybercartographic product [110].

In general terms, copyright law does not protect facts or information - only the original expression of that information [56, 122]. A map is considered an artistic work that is protected by copyright - it is a visual expression of facts or information [110]. Therefore, the fact/expression dichotomy applies to both cybercartographic products and the individual works (e.g., media) of which they are composed. Thus, the specific works that are protected by copyright law (e.g., a photograph) and the facts or information that are part of the work, are typically considered to be in the public domain [110].

A wide range of data protection and privacy laws exist - even within a single jurisdiction - that provide a level of privacy protection for individuals. Therefore, a cybercartographic product, which by definition is not confined to national boundaries, is potentially open to privacy complaints from multiple jurisdictions [110].

Personal information is generally considered to be any information about an identifiable individual [99]. The individual need not actually be identified - as it is enough if the information provided can be matched with other available information, so as to identify a particular individual. In our information society, there are so many available data sources and technological tools for searching and matching data that much information that is not obviously about an identifiable individual may well become so $[97]$.

Data protection laws generally prohibit the collection, use, or disclosure of personal 
information without an individual's consent. Expressed consent can also form part of a licence agreement where appropriate, such as a social media privacy policy. In many cases, privacy issues may not be significant. For example, data protection laws may not apply to non-commercial private sector activity [98]. There are also exceptions for data collected, used or disclosed for artistic, literary or journalistic purposes, although the full scope of these exceptions is constantly changing [96]. In the case where a cybercartographic product is hosted by a public institution such as a government agency or a university, public sector data protection laws may apply, and may place constraints upon how personal information is collected, used or disclosed [110].

Of course, some projects may inherently raise more privacy issues than others. Where, for example, the goal of a project is to invite contributors to report on illegal, antisocial, or simply controversial activities, there may be more specific privacy risks and potential liability - not just concerning privacy, but possibly also for other forms of civil liability [110]. The fact that privacy issues are inevitable suggests that cybercartographers will need to focus on what privacy regime applies to their work, and plan to manage the unpredictability of VGI within the context of that regime [110].

The synthesis of VGI and Cybercartography, despite the legal concerns described here, may well form the basis for a cross-sector solution to the two major challenges of the future of the Web, as identified by Sir Tim Berners-Lee [110]:

1. Linking large datasets on disparate topics (e.g., VGI), and

2. Displaying the resulting (new) information in innovative ways.

\subsection{Cybercartography for Education}

Teaching and learning are most effective when individuals are actively involved, as has been argued by educational theorists since Dewey's (1938) pioneering work [35]. Dewey's progressive learning theory is based on the idea that people, even very young children, are not blank slates waiting to be filled with knowledge from a formal education system. Instead, Dewey suggests that students organize fact-based comprehension through meta-cognition, or by building on prior experiences, preconceptions and knowledge, and therefore, he argues that the educator's role lies in creating an 
educative experience for the students [35]. In Nunavut, Canada, active involvement in the cybercartographic process is not limited to using cybercartographic atlases, but also represents the creation of atlas content, as part of the atlas creation process [110]. Cybercartographic atlases are highly interactive by design and often challenge users to interact with information visualizations in multiple ways. People use all of their senses when actively engaged in a learning activity. Cybercartography allows multisensorial representations of information that allow the user to engage with information in a format that is best suited to their learning style [110]. For example, Inuit society is an oral society and oral story-telling is of great importance to the preservation of traditional knowledge.

Many topics of interest to society are complex, and multiple ontologies or ways of knowing exist when considering the 'facts'. There is often no straightforward 'right' or 'wrong' answer to complex questions, or explicitly measured causal effects for global issues such as climate change. Cybercartographic atlases allow for the representation of a variety of opinions or narratives on such issues, rather than privileging one definitive answer over another [110]. The incorporation of traditional knowledge, especially regarding peoples' relationships with the environment are particularly important in Ingenious communities in northern Canada [110]. Intuit ontologies, or ways of knowing, differ from the academic peer review model, or a scientist's 'scientific method', and they must be considered as part of the atlas creation process if the resulting atlas is to have any utility to the community in an educational context.

\subsubsection{Cybercartography and Howard Gardner's Multiple Intelligence The- ory}

In his 1983 book, Frames of Mind, developmental psychologist Howard Gardner argued that students learn in very different ways and outlined seven 'intelligences' that all people exhibit to varying degrees. Gardner later added two additional intelligences; nine intelligences are included his most recent work. Gardner's nine intelligences are:

1. Linguistic intelligence: involves the sensitivity to spoken and written language, the ability to learn languages, and the capacity to use language to accomplish certain goals; 
2. Logical-mathematical intelligence: involves the capacity to analyse problems logically, carry out mathematical operations, and investigate issues scientifically;

3. Musical intelligence: entails skill in performances, composition, and appreciation of musical patterns;

4. Bodily kinaesthetic intelligence: entails the potential of using one's whole body or parts of the body (like the hand or mouth) to solve problems or fashion products;

5. Spatial intelligence: features the potential to recognize and manipulate patterns of wide space as well as the patterns of more confined spaces;

6. Interpersonal intelligence: denotes a person's capacity to understand the intentions, motivations, and desires of other people and, consequently to work effectively with others;

7. Intrapersonal intelligence: involves the capacity to understand oneself, to have an effective working model of oneself - including one's own desires, fears, and capacities - and to use information effectively in regulating one's own life;

8. Naturalistic intelligence: entails a person's ability to identify plants and animals in the surrounding environment; and,

9. Existential intelligence: entails a sensitivity and capacity to tackle deep questions about human existence such as, "What is the meaning of life?" "Why do we die?" "How did we get here?" [40, 41].

Gardner argues that educators tend to focus on teaching to the 'linguistic' and 'logical-mathematical' intelligences, and often neglect learners who exhibit the other seven forms of intelligence [41]. Hence, Gardner sees Multiple Intelligence (MI) theory as a basis for education reform, a reform that should encourage educators to engage learners using multiple intelligences. The principles that underpin MI theory closely resemble many of the core ideas of Cybercartography; however, Cybercartographic theory and practice was developed independently of the theory of MI until 2004, when 
the link with Gardner's work was made [9]. At present, Gardner looks at the applications of MI theory in educational contexts, and argues in favour of 'individualization' and 'pluralization' in educational settings:

put concretely we can approach topics in a number of different ways (often termed "entry points" to the same topic). We can make use of some of the analogies drawn from a range of domains and we can express the key notions or concepts in a number of different symbolic forms ... By pluralizing I mean that an educator should decide on which topics, concepts, or ideas are of greatest importance and should then present them in a variety of ways. Pluralization achieves two important goals: when a topic is taught in multiple ways, one reaches more students. Additionally, the multiple modes of delivery convey what it means to understand something well. When one has a thorough understanding of a topic one can typically think of it in several ways. Conversely, if one is restricted to a single mode of conceptualization and presentation, one's understanding (whether teacher or student) is likely to be tenuous [41].

Gardner's ideas fit well with the theory and practice of Cybercartography in relation to education, especially in the indigenous communities in Northern Canada. The most important concern when designing dynamic learning tools such as cybercartographic atlases is not the interactive technology, but the quality and relevance of the content contained in the atlases, as well as the processes by which that content is created [110]. A well-designed atlas does not equate to a well-designed teaching and learning tool if the content has not been thoroughly considered in terms of its relationship to the curriculum, and teaching and learning goals in both formal and informal educational contexts [110]. In Northern Canada, the involvement of Inuit communities in providing and determining relevant information to populate an atlas is of great importance. In Appendix C and Appendix D, we describe the practice of Cybercartography as it relates to the 'Inuit Siku (Sea Ice) Atlas' and the 'Arctic Bay Atlas'. 


\subsection{The Nunaliit Cybercartographic Atlas Framework}

The Nunaliit Cybercartographic Atlas Framework is an open-source web-based software development framework for interactive data management activities such as collecting, relating, and presenting and preserving information and its context [110]. Nunaliit is the underlying software application used in the creation of the cybercartographic atlases described in this project. In Inuktitut, the word 'Nunaliit' means community or habitat, and is itself a reflection of the principles of Cybercartography.

The Nunaliit Cybercartographic Atlas Framework began as an experiment in storytelling using interactive maps to display information, but has evolved into a highly flexible data management system with the ability to connect data and present information while maintaining context [16, 90, 109]. Many of the early cybercartographic products relied on curated collections of data and interactive atlas design to convey data-driven stories in dynamic environments, such as internet webpages.

Development of the Nunaliit platform continues to be driven by requirements outlined by its users in collaborative and iterative development processes that include software design and user-centred interface development, with a focus on capacitybuilding through ease of use [110]. Many cybercartographic products use datasets and information that has not been historically represented in mapping systems designed for broad audiences [110]. Many of Nunaliit's unique features and capabilities evolved as requirements to meet the needs of knowledge holders and researchers working in areas where existing tools and off-the-shelf software did not adequately meet user requirements. Recent development has focused on building interactive elements that are data driven but do not require restructuring for use in subsequent projects [110]. Using open standards, Nunaliit is designed to work with data stored in different locations, while permitting the creation of new relational connections that enable new stories to be told using the same underlying data. This fundamental principle distinguishes Nunaliit from other interactive story-driven mapping systems that feature embedded data structures in the application logic, and require development skill at the coding level to update maps with new information [110].

Data stored in the Nunaliit Cybercartographic Atlas Framework can be accessed

or edited directly by the end user - the prosumer of information. Nunaliit users are 
not required to navigate complex data structures, and instead have at their disposal a set of user-friendly database tools that permit queries, edits, and add and delete functions [110]. This is a significant difference relative to other popular open-source content management systems such as Drupal or MediaWiki, which are designed to broker all requests for information through application programming interfaces, although Drupal and MediaWiki do permit simple non-API user content addition. One of Nunaliit's core development principles is to avoid application-specific influences on raw data structures in order to encourage interaction with other systems [110]. Users with operational knowledge of Nunaliit are able to make significant changes to data structures, alter relationships between data, and render visualizations of information without programming skill [110].

The Nunaliit Cybercartographic Atlas Framework is developed and made available under an open-source license that permits reuse for any purpose. Specifically, Nunaliit is licensed under the BSD 3-Clause License which permits redistribution and use in source and binary forms, with or without modification, provided that the following conditions are met [110]:

1. Redistributions of source code must retain copyright notice, this list of conditions and the following disclaimer;

2. Redistributions in binary form must reproduce the copyright notice, this list of conditions and the following disclaimer in the documentation and/or other materials provided with the distribution; and

3. Neither the name of the copyright holder nor the names of its contributors may be used to endorse or promote products derived from this software without specific prior written permission [42].

Nunaliit works in collaboration with several other well-supported open-source technologies, and supports open standards for data use and data sharing where they exist [110]. Nunaliit uses standards ratified by the Open Geospatial Consortium, and other relatively new standards for geospatial information on the web, such as GeoJSON, an extension of JavaScript Object Natation (JSON) with support for geometries [110]. The Geomatics and Cartographic Research Center leads development 
of Nunaliit and is a long standing member of the Open Geospatial Consortium.

\subsubsection{The Evolution of Nunaliit}

The Nunaliit code base has undergone several significant changes over fifteen years of iterative development [110]. An overview of the early implementations of Nunaliit, including early user interfaces, major design challenges faced and discussion about how they were addressed, is available in Taylor (2014) [109]. Several highly relevant issues remain today, and some of these issues are discussed in the subsequent sections.

Data are different across a wide range of cybercartographic products. The relationships between data, the interaction model and data representations are also different, and require considerable resources to design and develop. The development of cybercartographic products by the GCRC and its partners requires significant resources in order to map relationships between data and develop custom code to address differences in information visualization techniques and interaction styles [110]. Much of the development cycle of cybercartographic products relies on data that is properly defined, and in the context of community-directed research, obtained. A reliance on outside data, often collected by community members, translates to delays in the iterative design process specifically related to user-interface design and data visualizations. In 2010, the Nunaliit development team transitioned the core database technology to CouchDB, a document-oriented database [3]. The implementation of CouchDB greatly improved the flexibility of Nunaliit as a whole, and increased its ability to host a wider variety of rapidly evolving atlases.

The Nunaliit development team designed an abstract module and schema concept to accommodate a wide range of data structures in cybercartographic products [110]. The metadata describes common data attributes and rendering options, and are stored in the database as documents, alongside but independent from, the data to be rendered. In Nunaliit, schema documents are metadata that describe what key:value pairs (attributes) to expect from a data document that self-identifies with that schema (i.e., it identifies the type of value expected and provides appropriate rendering) [110]. Possible value types include textual, numeric, or more sophisticated structures such as lists, dates, or requested custom structures. Module documents 
describe the display, interactivity options and data to be included in a particular rendering. The Nunaliit source code is able to store and parse schema and module documents, effectively separating the software from the specifics of a data document and its organization [110]. Atlas designers authorized to edit schemas and modules are able to significantly alter what is stored, how it is stored, and define rendering protocol, without programming knowledge. An operational goal of cybercartographic atlases is data accessibility via the user interface, without programming knowledge. To preserve data accessibility, the data must remain directly accessible via the user interface, without any requirement to go through Nunaliit or understand its schemas and modules [110].

The Nunaliit Cybercartographic Atlas Framework is used by several Indigenous community organizations, many of which do not have reliable access to high-speed internet and rely on satellite network services with slower speeds and higher latency [110]. Remote Indigenous communities have expressed a desire to host physical Nunaliit server systems within the bounds of their respective communities, as a means to retain clear sovereignty of their data and enable fast and reliable use of the Nunaliit system within the community. However, several challenges including a lack of robust server infrastructure and technical expertise needed to host and preserve active digital data systems within the community exist [110]. A potential solution to the data sovereignty problem is synchronized copies of an atlas, with one copy hosted in the community and a second copy hosted in a data centre with robust infrastructure and connectivity [110].

CouchDB permits multi-master replication and eventual consistency across multiple servers as core capabilities [3]. The Nunaliit development team was able to configure CouchDB to enable an atlas to be housed in multiple locations simultaneously and to synchronize data as network connectivity permits [110]. The custom setup enables community and data centre synchronous use, and also permits offline mobile instances of an atlas for field use when Internet connectivity is not available [110]. A central tenet of Nunaliit's design structure is its ability to effectively respond to changes in community needs and physical realities (e.g., internet connectivity and a community's technological ability to perform regular server maintenance, component 
upgrades, etc.) [110].

In the context of community mapping, data are often collected using several different technological mediums. A combination of digital cameras, audio recording devices, video recording devices, handheld GPS units, mobile phones and computers are often used in field observation work [110]. Further, a typical computer lab in a remote community is often not uniform in terms of operating system, configuration, or driver support. The diversity of equipment and available technological infrastructure in remote communities poses a significant challenge for community members and researchers alike, as it can contribute to lengthy documentation procedures and significant time learning multiple technologies [110].

Sharing content via online services, including social media, is a widely popular form of self-expression in many communities, and from a research perspective, it represents an opportunity to collect rich data insights in an information economy. High-speed broadband internet connectivity promotes instantaneous sharing of rich multimodal information, that is by definition, more accessible to a wider audience, relative to a unimodal information source, such as a voice recording device or pen and paper. There are many benefits to accessing social media via the API economy, however, we must consider the disadvantages of accessing social media data from large publicly traded technology companies such as Facebook and Twitter, among others, as well. From a research perspective, most social media data live in a closed system that users do not control and are only made available in a context and format set by the company [110]. When a technology company that maintains ownership and controls access to social media user data introduces incompatible changes to their internal data structure, or changes access protocols via APIs, researchers who are using the APIs can face uncertainty and may need tp pivot their work to accommodate new data access paradigms [110].

The Nunaliit architecture was developed to combine the best data collection approaches with server-side software under the atlas creator's control. The Nunaliit web interface permits consistent use in an online environment, and supports multiple types of data and enables offline field data collection and production [110]. Documents and their metadata, relationships, and user generated information can be synchronized to 
and from community owned and operated atlases that render information in a manner devised by the atlas creator [110]. All data contained in cybercartographic atlases remains under the control of the community at all times, and the system as a whole, including raw data and any surface level information visualizations, can be preserved and archived as required under the open-source BSD 3 Clause License [110].

\subsubsection{Recent Developments}

The Nunaliit Cybercartographic Atlas Framework is under active development by a small team at the GCRC at Carleton University in Ottawa, and to a lesser degree, at a handful of other institutions [110]. The full-time development team is typically focused on building out core functions as requested by community partners. A few recent developments, ranging from 2014 through 2019, are discussed below.

As described earlier, the shift to a related-document data approach with module and schema metadata was instrumental in freeing raw data from rendering decisions and software implementation. As the development team began to experiment with more advanced renderings, it also became important to combine data in more powerful ways, while simultaneously providing more powerful canvases, widgets and abstractions for atlas builders [110]. Nunaliit makes use of the 'layer' concept from GIS systems to group data documents, including those with mixed geometry types and data which contain no explicit spatial quality at all. In order to promote more powerful decision-making with respect to whether or not a document should be included in a set, and to increase control over the rendering of data, the development team opted to develop a new model of abstraction [110]. The added functionality allows an atlas user to alter system behaviour as needed, without assistance from a software developer, to re-adjust programming logic in order to make changes to data structures.

In a cybercartographic atlas, data can be described, stored, and accessed in its original form or a secondary form, with combinations, relationships, and other model and rendering operations performed by Nunaliit in a context-sensitive manner at the end user level [110]. The Nunaliit workflow is similar to the non-destructive workflow in photography or film editing, where the front-facing content is dynamically 
created according to a series of programmatic operations on the original raw photo or video files. The operations available to a cybercartographic atlas prosumer include adjustment, addition and deletion, re-ordered content, etc., while the system simply retains the original and a series of reproducible procedures [110].

In Nunaliit's early days, the development team focused on building mechanisms that enable exploration of relationships between data, as well as the production of easy-to-understand visualisations of related data. More recently, by underpinning Nunaliit with a document oriented database, data was liberated from homogeneous table structures, which required database administrators and programmers to implement routine structural changes [110]. Work on the multi-dimensional language web for the Atlas of the Languages of Iran, described by Anonby and Sabethemmatabadi, identified a new requirement [110].

The Nunaliit development team built a multi-dimensional language web to increase document flexibility and improve the representation of various languages spoken in Iran. Language structure is typically represented as a tree, with a hierarchy of parent and child languages; however, it quickly became obvious that a more relational representation would be most appropriate given the variation in language prioritization according to different subject experts and or language speakers. Deploying a user-centred design, a flat relational web design eliminated the requirement for a hierarchy and allowed the user to organize languages according to their specific needs and perspective [110]. The languages would remain related, but not all relations would be the same. Additional contextual information was necessary, and documentation and rendering served to define the language-language relationships. And with each language variety represented as its own document, independent webs could be documented in multiple separate language webs, which allowed an unlimited number of alternate (i.e., secondary and tertiary) representations. Nunaliit has been used to document various language classifications by different linguists, subset webs of language varieties where a specialist may only have knowledge of some of the web, and document contributions from local language speakers who may have very different ways of thinking about relationships between languages [110].

A Nunaliit module author is able to choose some or all of the web fragments 
to assemble a large rendering, or provide widgets for users to interactively adjust web fragments needed for rendering [110]. This was possible because the common pool of documents permits multiple semantic relationships, with different weighting. Weighting can be applied to visually strengthen relationships where there is consensus or to highlight outliers [110]. The ability to accommodate, store, render, and combine multiple perspectives and opinions is a key tenet of the practice of Cybercartography.

While many cybercartographic products can and often do have layers with geospatial features overlaid on base maps or imagery of the Earth, they are certainly not limited to GIS style representations. An overarching goal of good cybercartographic design is to hide extraneous data and tools from the end user and focus on optimal representations that convey understanding of a concept, pattern, relationship or story among the data [110]. The physical size, distance, or positioning of objects on a geographical map may not always be relevant to the user's story; and in some situations, it may be detrimental to remove valuable dimensions from the canvas available to a cybercartographer. Nunaliit is able to host custom Scalable Vector Graphics (SVG) deployed as interactive data visualizations, as were used in the early Antarctic and Trade atlases, in the Risk of Homelessness Atlas, and most recently, in the Inuktut words to images of sculptures (dubbed 'Sculptionary'), as well as in the night sky stories of the 'Pai Pai' in Mexico [109, 110].

Data-Driven Documents (i.e., D3) is a JavaScript library for manipulating documents based on data. D3 helps bring data to life using HTML, SVG and CSS, and its emphasis on web standards provide the full capabilities of modern browsers without tying the developer to a proprietary framework [15]. D3 combines powerful visualization components, with a data-driven approach to DOM manipulation, and is accessible under an open-source license similar to Nunaliit [110]. The Nunaliit development team built a D3 map canvas to implement data visualizations. Using D3 visualizations, a user can select or hover over an item in the visualization and trigger audible sounds, and activate or highlight documents in a list, etc. The D3 integration is active in the Ontario Brain Institute's AxON system [110].

Desktop GIS systems typically consist of expensive workstation-grade computing systems with high performance memory, processing, graphics, and storage capacities 
that enable timely rendering of complex vector geometries and raster imagery on screen. In contrast, a typical web browser on a consumer-grade personal computer is constrained in many of the above capabilities and struggles to render geospatial datasets, particularly the complex vector data with which a user is expected to interact [110]. The technological capacity of computing devices is a serious barrier in terms of prosuming cybercartographic products in remote communities with limited technological resources. To address some of the technological challenges, the Nunaliit development team redesigned the original source code to store original geometry as an attachment, alongside a configurable number of additional simplified versions to be rendered as befits the zoom level of the map [110]. Similar to email attachments, the additional files can be connected to a document, but are not necessarily shown or even retrieved until requested (i.e., via zooming or selecting a linked document to uncover new documents, etc.) [110]. Amos Hayes (GCRC) describes the process as:

"The document itself simply contains a point geometry representing the centroid. Now when Nunaliit is first asked to render documents containing geometries on a map, it first draws them in as points and then quickly works from the centre of the map outwards to determine if any of the geometries have a more detailed version that warrants being displayed at the current zoom level. If so, it replaces the point with that more complex version. As the map is zoomed in or out by the user, Nunaliit repeats this process to ensure that the map is rendered with the most appropriate version of the geometry for the scale. This has enabled Nunaliit to greatly improve responsiveness while simultaneously representing tens of thousands of complex features on a map." [110]

Clustering is another design technique often employed in complex cybercartographic products to minimize the number of point features rendered, and is used to improve usability when large numbers of point features are geo-located in close proximity. In many cybercartographic atlases, line and polygon-based geometries can be clustered when they are too small to interact with at a given scale (e.g., zoom-wise or due to a lack of visual display area). Clustering permits complex mixes of points, lines, and polygons on the same map canvas without sacrificing usability at smaller 
scales [110].

A common challenge in the creation of cybercartographic atlases is the process of bringing outside multimedia information into an online atlas. In several atlases co-produced by the GCRC team and its community partners, adding multimedia content, such as video and auditory files, is a challenge [110]. Issues can arise when a multitude of data collection devices to used, but also when a lack technological infrastructure exists, especially in remote communities, such as fly-in communities in Northern Canada, where travel equipment must be kept to a minimum [110].

The Exchange for Local Observations and Knowledge of the Arctic (ELOKA) team at the US National Snow and Ice data Centre operate several Nunaliit instances in partnership with communities in Alaska and actively contribute to Nunaliit's development. Using WebRTC standards implemented in modern browsers, ELOKA developers improved Nunaliit's file uploading capacity to include in-browser video and audio recording and compression [110]. As a result, Nunaliit users are now able to record, preview, and formally upload audio and video content within an internet browser. During the upload process, the user is prompted to input any additional metadata based on the underlying schema. All uploaded media is stored as is before being processed by Nunaliit and stored with uniform specification (e.g., size, resolution, codec, bitrate, etc.), determined by the Atlas administrator [110].

\subsubsection{Future Development of Nunaliit}

The Nunaliit Cybercartographic Atlas Framework continues to be iterated through numerous sources of research, heritage and community funding, and remains focused on meeting the needs of ongoing community projects. As new functional elements are added to the code base, the development team is always considering ways to implement features that enable broad use and access across the international Nunaliit community [110].

Joint development of the software, along with education, training materials and community programs, has helped to establish a diverse team with similar values, which in turn facilitates the production of high-value cartographic products. International community partnerships have led to increased accessibility through an 
increasing number of training materials and translation of the original English documentation into Spanish, and most recently, Portuguese [110].

Recent developments continue to focus on making it easier to tell better stories with rich multimedia information, using interactive multimodal interfaces, while maintaining time and space representations with robust data management concepts that continue to be built into the system [110]. The process of building an atlas, working through all the decisions such as: what information is relevant and to whom; what relationships between the data are relevant and to whom; and, how should data be processed and stored for optimal accessibility and reusability by future prosumers of cybercartographic products, are all relevant and ongoing questions Cybercartographers around the globe are working on in an effort to advance the theory and practice of Cybercartography. The more relevant, usable, learnable and accessible cybercartographic products are, the greater the opportunity for representation grows. 


\section{Chapter 3}

\section{Methodology}

\subsection{Timeline}

We describe our work in three phases; Phase 1: Field Observations, began in September 2019 and concluded in December 2019; Phase 2: Participant Identification, Recruitment and the Semi-Structured Interview, began in February 2020 and concluded in April 2020; Phase 3: Prototype Build and the Usability-Testing Session, began in September 2020 and concluded in April 2021.

\subsubsection{Navigating the Covid-19 Global Pandemic}

The first confirmed case of Covid-19 in Ottawa was reported on March 11, 2020 [93]. On March 17, 2020, Premier of Ontario, Doug Ford, declared a Provincial State of Emergency in response to the ongoing Covid-19 Global Pandemic, and we made an independent decision to immediately pause interaction with the community until further direction was provided by the Carleton University Research and Ethics Board (CUREB) [5]. The decision to pause our research was made in an abundance of caution, as we were in the process of conducting semi-structured in-person interviews after receiving ethical clearance to begin participant recruitment on February 20, 2020. The Provincial State of Emergency remained in place for several months, was rescinded for a short period of time, and was re-instated and remained in place as of June 2021. Due to the prolonged province-wide State of Emergency, we made two significant pivots in our work. First, we modified the cybercartographic atlas design procedure due to restricted access (i.e., emergency only) to the Geomatics and Cartographic Research Center and Nunaliit development team at Carleton University. Second, we built a low-fidelity prototype (in place of an actual Cybercartographic Atlas with minimal functionality or high-fidelity prototype), that was evaluated in 
remote usability-testing sessions, as described in chapter 4. While considering prototyping options (i.e., high-fidelity vs. low-fidelity), we chose to build a low-fidelity prototype because we were aware that multiple participants may not have access to an iPad Pro, high-speed broadband internet, or a similar computing device with sufficient resources to run a high-fidelity prototype or web-based resource intensive cybercartographic atlas.

\subsection{Phase 1: Field Observations}

We passively participated in Town Halls, City Council and Transit Commission meetings, visited major transit hubs during peak-service hours, and observed the public transit conversation happening on Twitter. The purpose of the field observations was twofold: first, we hoped to learn about issues of importance to public transit users in Ottawa, and second, we hoped to document any meaningful differences in terms of what and how public transit issues are discussed in different formal and informal venues. As we conducted field observations, we noted several barriers to access the public transit decision-making process, especially among members of the PWD community.

\subsubsection{Town Halls}

We attended Town Halls in three Ottawa neighbourhoods and passively observed the interactions and discussions that took place. Our passive observations included learning about public transit issues as described by the community, and documenting the structure of the discussions that took place. We noted venue setup (e.g., panel discussion, round table, opportunity for public delegation, etc.), the nature and tone of the conversation (e.g., moderated discussion, organized participation, disorganized and chaotic, etc.), and we recorded publicly available contact information from attendees (e.g., panellists, organisers, keynotes, elected and or appointed officials, community members, etc.), which was used to populate a participant recruitment pool. 


\subsubsection{City Council and Transit Commission Meetings}

We attended multiple Ottawa City Council and Transit Commission meetings, both in-person and virtually via the City of Ottawa YouTube channel. We noted venue setup (e.g., in-person public delegation and media were permitted in the gallery, while elected and appointed officials received access to the main concourse), the nature and tone of the discussions (e.g., organized participation, structured and declarative speech for discussion of official motions, enforced time limits for participation in the discussion of agenda items, including formal public delegations, etc.). Specifically, we noted opportunity for public delegation at the Transit Commission, if requested twenty-four hours in advance of the scheduled start time of the meeting, and under exceptional circumstance, at regular City Council meetings. While observing the Transit Commission meetings, we noted available mediums for public delegation: inperson speech from the speaker's box (microphone activation for virtual meetings), an ability to have a written statement read aloud in a delegate's absence, and capacity for audio/video content (if arranged in advance) to be broadcast for the committee using the City of Ottawa's in-house technology setup. Further, we noted several sidebar discussions that took place during in-person meetings at Ottawa City Hall. The sidebar discussions often took place outside the scope of the camera, and were often not represented in digital uploads to the City of Ottawa YouTube channel.

\subsubsection{Major Transit Hub Site Visits}

We observed morning and afternoon peak-service at several major transit hubs along the Confederation Line. During peak-service hours, we noted the volume of public transit users attempting to traverse bus platforms, the volume of public transit users idling on the platforms, and availability of accessible infrastructure (e.g., wayfinding, electrified doors, service elevators, ramps, winter-maintained stairs, public restrooms, etc.). Our goal was to observe public transit users' interactions with the built environment at major Bus/LRT (Light Rail Transit) stations during peak-service hours. 


\subsubsection{Social Media Observations}

We passively observed several dozen social media interactions concerning public transit service and or public transit infrastructure on the social media platform, Twitter. We noted active accounts, the nature of interactions, the inclusion of multimedia in user posts, the use of 'hashtags' to organize information, and the use of '@citizen' to tag a specific audience, namely, various media organizations, public transit advocate/lobby groups, elected and or appointed officials, and the local public transit authority.

\subsection{Phase 2: Participant Identification, Recruitment and the Semi-Structured Interview}

Throughout Phase 1: Field Observations, we observed the public transit conversation from multiple perspectives. In many instances, citizens voluntarily shared their given name when speaking at Town Halls, while given and surnames were provided to meeting organizers for public delegation at Ottawa City Hall (e.g., Transit Commission and City Council Meetings, including virtual meetings). The provision of explicit personal identification information is a condition of participation as a public delegate at Ottawa City Hall [28]. We established a list of eighteen potential participants from our passive observations in formal and informal public venues that

hosted public transit discussions, and from our social media observations. We googled the eighteen prospective participants and identified social media profiles, professional networking profiles, and personal websites with additional contact information (e.g., email, phone, etc.).

\subsubsection{Participant Identification and Defining Stakeholder Groups}

We sorted the participant pool into the three public transit stakeholder groups, based on our observations from formal and informal public transit discussions. Prospective participants were informally assigned to a public transit stakeholder group based on how they chose to self-identify while they were passively observed during the field 
observation phase. Prospective participants that were contacted and invited to participate in the study were screened using a screening questionnaire, and asked to self-identify as belonging to one of the three public transit stakeholder groups we identified, in addition to self-identifying as an active Twitter user. Further, participants were asked to self-identify a second time at the beginning of the semi-structured interview. All participants were made aware of the three public transit stakeholder groups participating in the study during the semi-structured interview. The three public transit stakeholder groups in this study are:

1. Persons who self-identify as living with a disability or experiencing additional barriers to access public transit infrastructure and decision-makers;

*We refer to this group as the 'PWD community'

2. Persons affiliated with public transit advocate/lobby groups; and, *We refer to this group as the 'public transit advocate/lobby community'

3. Persons elected or appointed to the Transit Commission, thereby holding voting rights at the public transit decision-making table.

*We refer to this group as the 'elected, and or appointed official'

\subsubsection{Participant Recruitment}

Following CUREB-B clearance, we began contacting participants using public-facing contact information retrieved from social media profiles, professional networking profiles, and personal websites. Our initial goal was to recruit three participants for each of the three stakeholder groups (nine participants in total) to participate in the study. We made a concerted effort to recruit visible minorities from marginalized groups that are historically under-represented in terms of access to accessible public transit infrastructure and access to the public transit decision-making process. We believe that a diversity of opinion is important for community representation. We deliberately chose to engage with visible minorities and marginalized groups who hold opinions and beliefs about accessible public transit infrastructure that are not always reflected in public transit decision-making. All prospective participants were provided with a formal 'Letter of Invitation' and an initial 'Screening Questionnaire', via 
email. Six individuals, representing three public transit stakeholder groups, agreed to participate in the semi-structured interview.

\subsubsection{The Semi-Structured Interview}

We curated three variants of the semi-structured interview - one for each public transit stakeholder group. Each semi-structured interview had three sections:

1. A brief introduction (participants were encouraged to self-identify any physical disabilities or additional barriers they encounter while using public transit infrastructure, as well as describe a hobby or job - where applicable);

2. Understanding a participant's experience using public transit infrastructure (both physical and digital infrastructure); and,

3. Understanding a participant's experience using the social media platform, Twitter, to converse with other users about public transit issues.

Each participant actively took part in one thirty-minute semi-structured interview. The process of the semi-interview is best described as a conversation in which the participant was encouraged to speak candidly about their day-to-day use of the public transit system, and their use of the social media platform, Twitter, to discuss public transit issues with other users. The semi-structured interview took place in a mutually convenient public location (e.g., a coffee shop, public library, art gallery, etc.). All subsequent communication with participants, namely, the usability-testing session, was conducted remotely using the Zoom web application.

\subsubsection{Establishing Stakeholder Mandates}

The semi-structured interview was audio-recorded with the participant's consent. The audio recordings and research notes from the semi-structured interview was transcribed, and is described in detail in chapter 4 . The interview guide for each of the three participant groups is included in Appendix E. The results of the semistructured interview were used to establish a mandate for each of the three public transit stakeholder groups. The semi-structured interview was designed to answer two 
core research questions, and additionally served to define the existing public transit decision-making IS:

1. What are the needs of the PWD community in terms of access to public transit infrastructure and access to the public transit decision-making process? What barriers to access exist, and what can be done to reduce the number of barriers?

2. How must the existing public transit IS change in order to incentivize community narratives over intuitive judgements, at the policymaking level?

The information gathered during the semi-structured interview was grouped by stakeholder, and merged to define the existing public transit decision-making IS. We were able to map the bi-directional flow of information between the PWD community, the public transit advocate/lobby community and the public transit decision-maker.

\subsection{Phase 3: Prototype Build and the Remote Usability-Testing Session}

Initially, we planned to build a proper cybercartographic atlas (with limited functionality) that served two core functions:

1. Permit a user to query a database populated with Twitter data; and,

2. Permit a user to actively interact with multiple representations of the query results, plotted temporal-spatially where appropriate, and additionally visualized in an information timeline, adjacent to a cybercartographic atlas.

We pivoted our work to reflect available resources and remote usability-testing constraints during the Covid-19 Global Pandemic. We used 'figma', a vector graphics editor and prototyping tool which is primarily web-based, to build a low-fidelity prototype in lieu of a cybercartographic atlas with limited functionality.

\subsubsection{The Prototype Build}

Early paper-based sketches were modelled after the Advanced Google Search tem-

plate, as shown in Figure 3.1. Google Search is by far the most popular worldwide 
search engine, and the Advanced Search template provides a good starting point for database query design. Notably, we maintained the Google database query page structure, including the use of two headers to separate query input fields on the right hand side and the 'To do this in the search box.' header, with example text, on the left hand side of the Database Query page. The centre of the Database Query page was populated with data entry fields, as shown in Figure 3.2. This design solution does not require special knowledge or instruction, and is consistent with the design principles discussed in subsequent sections.

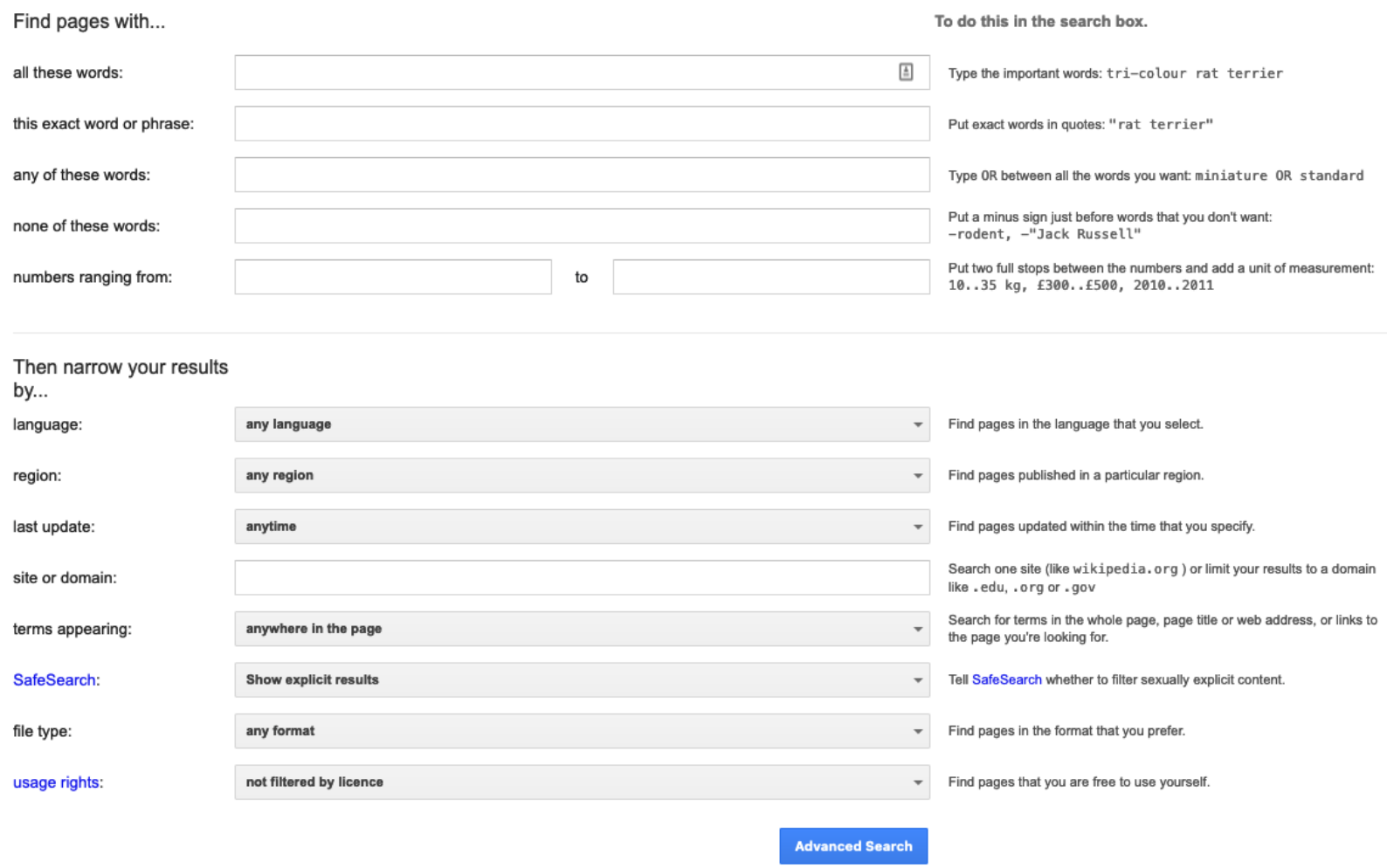

Figure 3.1: Advanced Google Search Template 


\section{Home > Accessible Public Transit Advocate}

Welcome to the Accessible Public Transit Infrastructure Atlas-ACCESSIBLE PUBLIC TRANSIT ADVOCATE view! The information presented in this atlas was harvested from the social media platform, Twitter, and is plotted spatio-temporally on a GIS style map. The map is interactive by design, and includes audio-visual media, URLs, and up to 280 characters of text. You may interact with content by selecting an icon on the map or by scrolling the tweet timeline. We are actively working to geolocate as many tweets as possible.

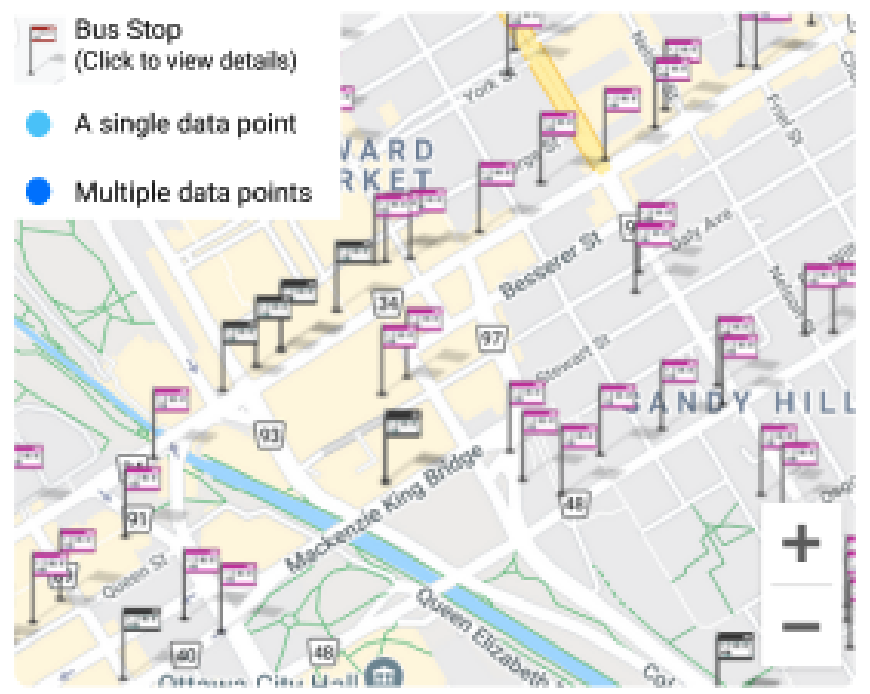

Find tweets with...

all of these words

none of these words

all of these hashtags

all of these accounts

Then narrow your results with...

date range

media attachments

location
To do this in the search box.

e.g. word1, word2, word3

e.g. -word 1, -word 2

e.g. \#PWD, \#ParaTranspo

e.g. @CTVnews, @CBC

To do this in the search box.

e.g. YYYY-MM-DD

e.g. slect all that apply

e.g. 3052 or Parliament

\section{Search}

Figure 3.2: Empty Database Query 
We designed a series of wireframes with $100+$ unique components, and linked the wireframes sequentially to produce a low-fidelity prototype, with a mock cybercartographic atlas as the central artefact, as shown in Figure 3.3 and Figure 3.4. We defined two use cases to evaluate select functional and non-functional requirements. The primary functional requirement we evaluated was a participant's ability to conduct a database query and navigate to the Query Results page, and the primary non-functional requirement was to do so without explicit instruction from the researcher (i.e., the participant was expected to determine the appropriate location to input query criteria and then determine the appropriate button to navigate to the Query Results page).

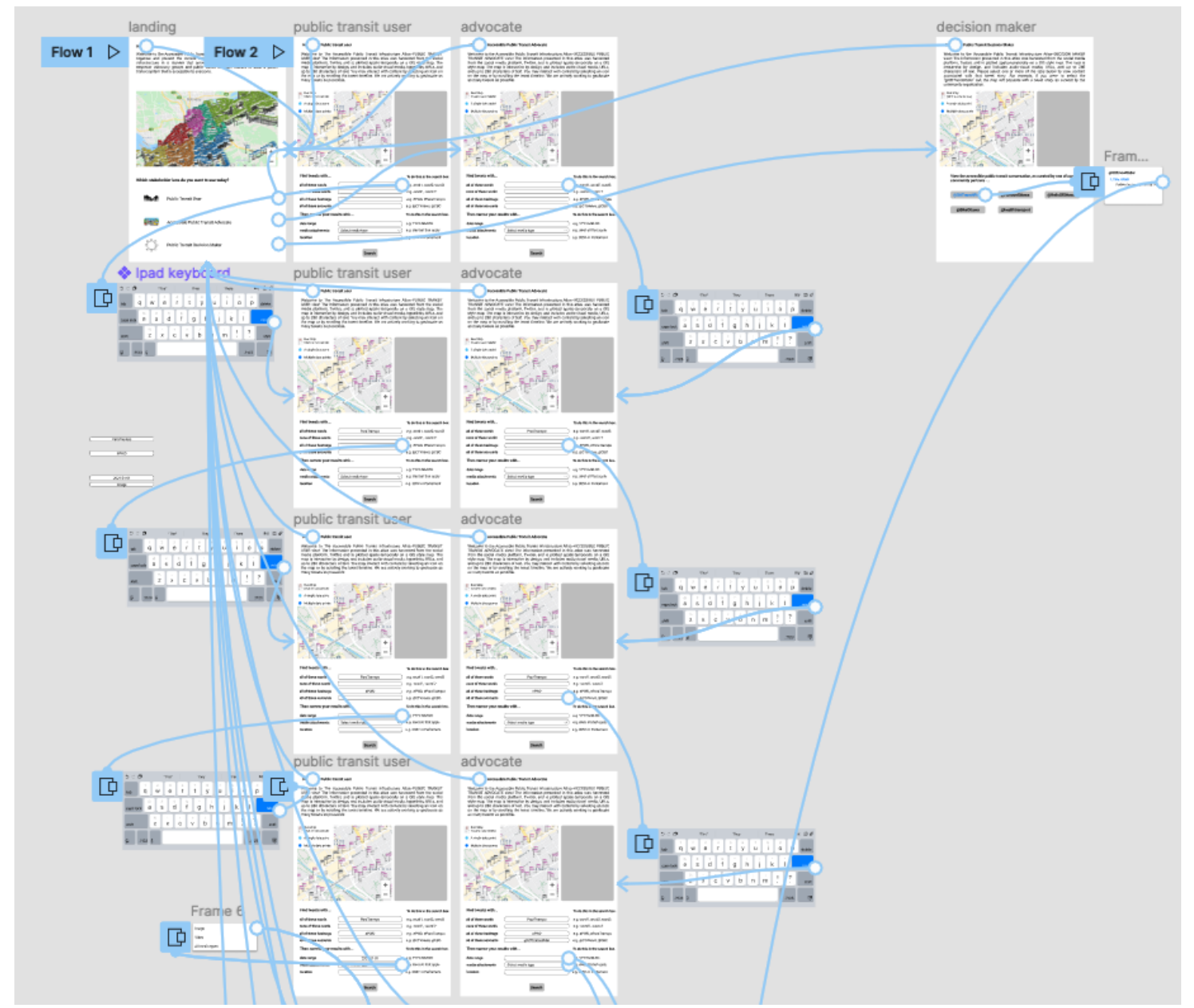

Figure 3.3: Prototyping Query Flows 


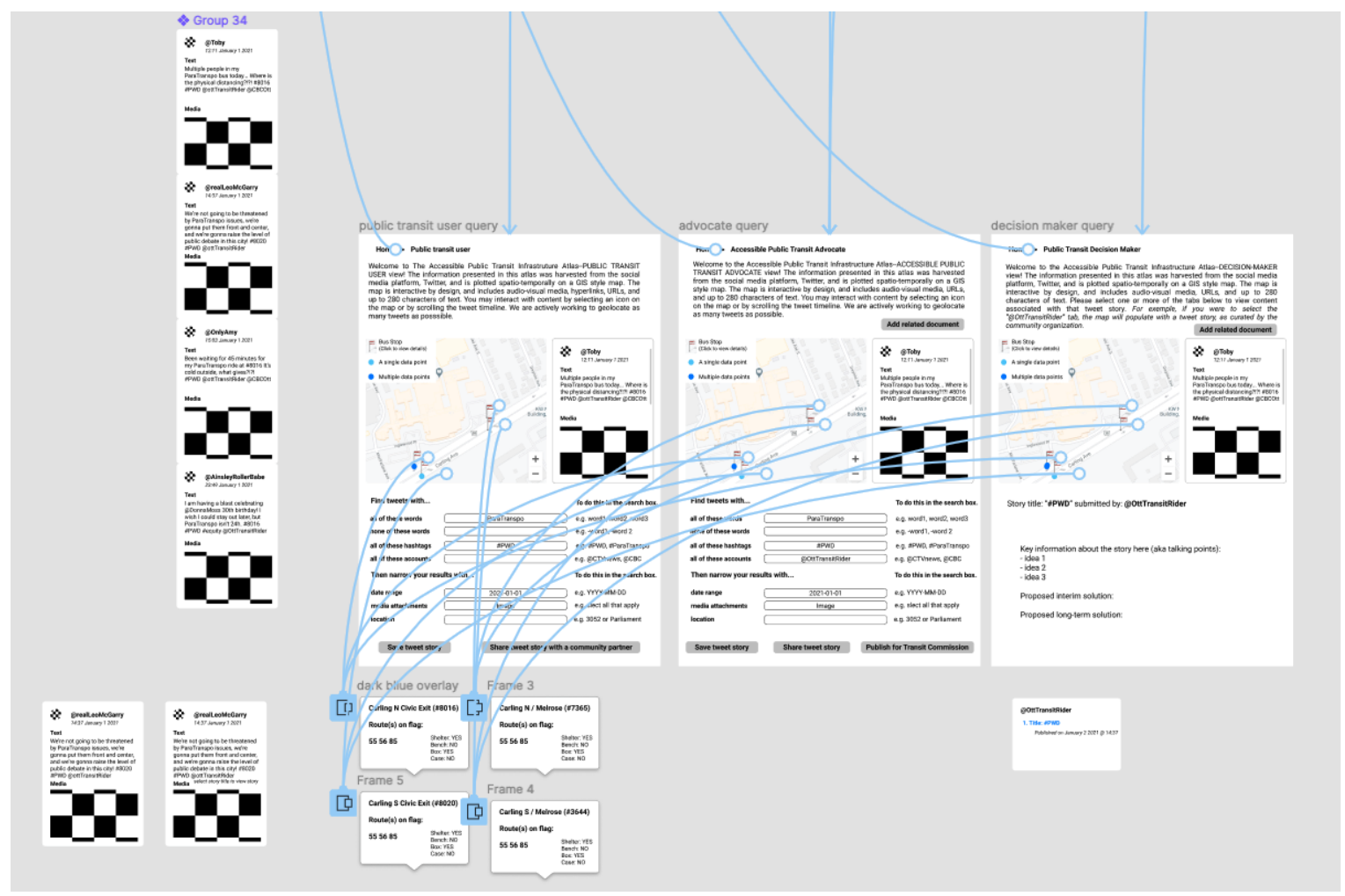

Figure 3.4: Prototyping Result Flows

Prior to evaluating the database query use case, participants viewed and explored the functionality of a 'Landing' page, and selected a user profile that represented their status as: a public transit user, a public transit advocate/lobbyist, or a public transit decision-maker. The primary functional requirement we evaluated was a participant's ability to recognize information embedded in the GIS style map and recognize the intended function of the zoom icon. The primary non-functional requirement we evaluated was a participants ability to identify three user profiles and select the user profile that best described their status, in order to navigate to the Database Query page.

Once a participant had conducted a database query and navigated to the Query Results page, they were encouraged to explore the results of the query in detail. Query results were displayed temporal-spatially in a mock cybercartographic atlas, and additionally in an adjacent information timeline. Each participant group evaluated the same two use cases; however, the user interface for each of the Database 
Query and Query Results page was designed to reflect a stakeholder group's unique needs in terms of query functions and result visualizations. I.e., three custom user interfaces were designed, one for each stakeholder group, and each interface design included modified user permissions, query and visualization tools.

\section{Understanding the Design Space}

Cybercartographic products are, by definition, interactive and where possible, use all five senses in their representations of information, often employing multimodal approaches to convey important information [110]. In contrast, a low-fidelity prototype is a first, typical or preliminary model of something that is incomplete, and has limited functionality relative to a fully functional piece of software. In consideration of the contrasting forces of cybercartographic products and low-fidelity prototypes, we chose to evaluate a simple database query task and results exploration task that leveraged a core strength of Cybercartography - namely, to show multiple representations of temporal-spatially referenced information for users with different informational needs.

We observed participants perform a standard database query, and then proceed to explore the mock cybercartographic atlas and adjacent timeline information visualizations, both of which were populated with mock Twitter data. We made a deliberate effort to simulate multimedia content by employing chequered image placeholders where appropriate, and we included various interaction elements such as buttons, scroll bars and hyperlinks in the interface design. We recognize the limitations of remote usability-testing a tablet-based prototype on a variety of technological mediums (e.g., tablet, laptop with trackpad, desktop with keyboard and optical mouse, etc.) - i.e., the remote usability-testing sessions took place on various technological devices and participants completed the tasks using various interaction techniques. In some instances, participants completed the usability-testing session by selecting a web link which displayed the prototype on their native device using an iPad Pro 11 (834x1194) frame, while other participants used gesture-based interactions to complete the usability-testing session on a tablet interface. 


\section{Design Principles and Philosophy}

In consideration of the constraints of remote usability-testing and researcher dependency on a participant's ability to configure the Zoom web application to enable 'screen share', in addition to audio/video communication, and further, to configure their internet browser settings to open and interact with the prototype, we designed our prototype with three core principles in mind:

1. Do not make the user think about the interface itself;

2. Do not build resource intensive wireframes and interactions (i.e., keep graphical content, resolution, and other resource intensive activity to a minimum); and,

3. Make buttons and navigation obvious, purposeful, and accessible on a wide range of possible user interfaces (i.e., on multiple technological mediums with different interaction styles and varying screen real estate).

The ultimate evaluation of a web-based user interface is whether or not a user can view the interface for the first time and say "Oh, when I select this, 'XYZ' will occur'”, or "OK, there's the button for 'X", and "Ah, that's an 'input field" and "There's the button I need to accomplish 'Z'". The user should be able to 'get it' - what it is and how to use it - without expending any energy thinking about it [66]. We used a 10-item questionnaire with five response options for respondents, from Strongly agree to Strongly disagree, to evaluate perceived system usability. The System Usability Scale (SUS) was developed by John Brooke in 1986, and is considered the industry standard for quick assessment of perceived system usability, with over 11000 citations in peer-reviewed publications [18]. Some benefits of using the scale as an evaluation metric include:

- It is a very easy scale to administer to participants;

- It can be used on small sample sizes with reliable results; and,

- It is valid-it can effectively differentiate between usable and unusable systems $[18,19]$. 
The second core design principle is a requirement to design a non-resource intensive prototype. Remote usability-testing can provide the research and development team with insight from near and far (geographically), provided the interaction medium, access to high-speed broadband internet, the central processing unit (CPU), randomaccess memory (RAM), the graphics processing unit (GPU), and a participant's level of technological skill conform to a predetermined baseline measure. In this study, we conducted remote usability-testing with multiple participants who reported living below the poverty line, and thus, did not have access to an iPad pro, high-speed broadband internet, and in one instance, to a personal computing device. We do have access to an iPad Pro, and initially planned to provide all participants with access for the purpose of usability-testing, however, in-person usability-testing was not possible, due to the Provincial State of Emergency and stay at home order. Accordingly, we made a deliberate effort to build a non-resource intensive prototype that could be deployed on a variety of technological mediums, with varying interaction styles. We achieved a baseline level of accessibility by eliminating audio/video content, restricting prototype functionality to the evaluation of two relatively simple use cases, and most importantly, designed a tablet-based interface with gestural interactions, that is also accessible to an individual using a trackpad or optical mouse.

The third core design principle is a requirement to make navigation obvious, purposeful, and accessible on both tablet, and mouse and keyboard systems, with different amounts of screen real estate. We addressed this requirement by employing well-established patterns for interactive design, as described by Jenifer Tidwell in Designing Interfaces: Patterns for Effective Interaction Design [115]. Notably, we imported an 'iOS/iPadOS 13 UI Kit' in the figma web application, which provided access to common assets such as: Bars (e.g., Home, Search, Navigation, Status, etc.), Controls (e.g., Buttons, Date Picker, Text Field, etc.), System Elements (e.g., Keyboard, Widgets, etc.), and Device Bezels. The availability of a pre-defined asset library allowed for quick development of a low-fidelity prototype, with cross-device interactivity and scalability, while employing a common UI system that does not require significant $\mathrm{CPU}$ processing power, graphical rendering, or high-speed internet bandwidth. By deploying a tablet-based prototype, we maintained an ability to scale 
the UI across mobile, tablet, and desktop applications with various resolutions and screen real estates.

\section{The Collection, Organization, and Visualization of Twitter Data}

With Twitter API, a licensed Twitter developer is able to tap into the public conversation to understand what's happening, discover insights, identify trends, and much more [121]. The high volume of data, in a variety of media, creates a significant advantage when building a dataset using Twitter API. Building a high-quality filter ensures that the data a developer is able to access is sufficient for their use case, and representative of the conversation they are looking to analyse. Moreover, building a proper filter can significantly reduce the time and effort required to clean a dataset, and ensure that data access limits are respected [121]. Our initial methodology, prior to the Covid-19 pivot, included a process in which the semi-structured interview would be used to inform the development of a high-quality filter to access the public transit conversation happening on the platform, and pull it into the Nunallit Cybercartographic Atlas Framework, where the data could be organized by the public transit advocate/lobby community to create information for other community partners and public transit decision-makes alike. I.e., the accessible public transit conversation happening on Twitter would have enabled the research team to build a high-quality filter, in order to access raw twitter data for the explicit use case: learn about the current conversation re: accessible public transit infrastructure in Ottawa.

During the semi-structured interview, participants were asked to describe their Twitter interactions, including the use of handles (i.e., user accounts), hashtags, keywords and slogans, while participating in the public transit conversation happening

on the platform. Participants were also asked about the inclusion of various media attachments, in addition to text-based tweets, in their interactions. This information was gathered during the semi-structured interview (prior to the onset of the Covid-19 Global Pandemic), and was intended to be used in the development of a high-quality data filter. Raw Twitter data is stored as JavaScript Object Notation (JSON), a format that is compatible with the Nunaliit Cybercartographic Atlas Framework's related document structure. While, we acknowledge that building a high-quality data 
filter is not a simple exercise, we planned to build a modest filter using a combination of hashtags, accounts and keywords, derived from the semi-structured interviews, to produce a minimal working example (MWE), to be evaluated in an initial usabilitytesting session.

The foundational questions of "Why", "What", "When", "Who" and "Where" define what data a researcher can access via Twitter API. Of particular importance to the practice of Cybercartography, the questions of "When" and "Where" enable the transformation of raw Twitter 'data' into temporal-spatially referenced 'information' in a cybercartographic product. Geo-locating non-geotagged tweets to show temporalspatial relations among data is a major challenge in this project. We conceived prospective solutions to address the challenge of geo-locating raw twitter data with a two step process:

1. First, we would include a public transit infrastructure map layer (available to licensed developers via the local public transit authority or Open Ottawa) that identifies the geographic position of all public transit infrastructure with a unique four digit identification number (a unique identification number is currently posted on all pubic transit infrastructure); and,

2. Second, we would use the unique four digit infrastructure identification number to geolocate raw Twitter data that presented with an infrastructure number embedded in the tweet (e.g., If a tweet contained: "Bus stop \#1000 is my favourite stop in the city!", the tweet could be automatically plotted temporalspatially according to the attributes defined in the infrastructure layer). In addition to the infrastructure identification number, we could begin to define additional identification attributes in the infrastructure layer (e.g., proper place names for major transit hubs: 'Parliament Station' or Bus Stop \#1000.

Finally, we include a secondary representation of the public transit conversation by employing a well-established timeline visualization, adjacent to the central artefact, the cybercartographic atlas. The implementation of a mock cybercartographic atlas and an adjacent timeline visualization provides an opportunity to include nongeo-located tweet data. A central tenet of Cybercartography is the presentation of 
information in different formats, using different ontologies or narratives, without privileging one over another [110]. In our prototype, the public transit conversation is represented temporal-spatially in a mock cybercartographic atlas, and additionally, in an adjacent interactive information timeline. Thus, a user can choose to engage with temporal-spatial information visualizations, or familiar timeline information visualizations.

We identified three public transit stakeholder groups in the course our field observations. From the stakeholder groups, we determined two information visualization needs:

1. The public transit user and the public transit advocate/lobby community require information visualizations that are conducive to knowledge generation first, and that serve to improve judgment, second; while,

2. The public transit decision-maker requires information visualizations that promote effective decision-making first, and that are conducive to knowledge generation, second.

Educational theory suggests that individuals learn best when they are actively, rather than passively engaged [41]. Cybercartographic atlases have great potential in both formal and informal education as they promote interaction with information representations using multiple lenses and perspectives to facilitate a holistic user experience [110]. The design of the Landing page allows a user the freedom to selfdirect to an appropriate profile. Each user profile presents information visualizations in a mock cybercartographic atlas, as well as an interactive information timeline. The information visualizations in each user profile are generated from a common dataset, although user interactions and data permissions vary for each user. For example, the public transit lobby/advocate user profile includes an option to 'Add a related document' and a button to 'Publish for Transit Commission', while the decisionmaker user profile presents information visualizations in a mock cybercartographic atlas, an interactive timeline, and has an additional section with three bullet points of text indicating key information about the query results (i.e., tweet story), authored by a public transit advocate/lobby group. The three bullet point section provides an 
opportunity for community experts - for example, the president of a local architecture firm - to communicate with decision-makers about the built environment in language that is accessible to the city planners and engineers responsible for the Strategic Asset Management Plan.

\section{Challenges}

Taylor (2019) identifies several challenges associated with accessing social media data via the API economy. While we agree that the costs of accessing social media data can be prohibitive under the current Nunaliit Cybercartographic Atlas Platform business model, both in terms of pay-for-access data fees and the in-house development resources needed to build and maintain high-quality data filters, we believe that the practice and process of Cybercartography remains challenged to adapt to the user-centred social media revolution in novel ways. We believe that the practice of Cybercartography should continue to be applied at the community level, and we consider the possibility that cybercartographic products may one day leverage the API economy, not necessarily as a core data source, but as a supplement to the core ideas and learnings realised through iterative community dialogue.

\subsubsection{The Remote Usability-Testing Session}

We amended the initial usability-testing procedure to comply with the ongoing Provincial State of Emergency. Participants took part in remote usability-testing sessions using the Zoom web application. Participants were asked to configure their native technological device in the following manner:

1. Accept a Zoom meeting invitation, via email;

2. Join the Zoom meeting by selecting a hyperlink provided via email, and enable 'audio/video sharing';

3. The participant was encouraged to close any background applications that may contain personal information (e.g., email, messaging, etc.);

4. Disable 'video sharing' and enable 'screen sharing' in the Zoom web application; 
5. View the Zoom chat, and select a hyperlink to access the prototype;

6. Complete two use cases during the usability-testing session;

7. Complete a 10-question post-test questionnaire (SUS), and returne the questionnaire via email;

8. Provide a mailing address for contactless delivery of the $\$ 20$ gift card compensation.

\section{Stakeholder Group 1}

Participants who self-identified as a member of the PWD community completed two use cases during the remote usability-testing session. Following evaluation of the use cases, the participant was provided with an opportunity to pose questions, and was asked to complete a post-test questionnaire and return it via email. After the participant returned the post-test questionnaire via email, they were asked to provide instruction for contactless delivery, or a mailing address to facilitate delivery of a "Thank You" card enclosed with a \$20 Tim Horton's gift card, as compensation for their participation in the study.

In Use Case 1, the participant was asked to view and explore the functionality of the Landing page. We guided the participant using pre-determined, open-ended questions, adapted from Steve Krug (2009):

- "What are you looking at?"

- "What are you thinking?"

- "Is this what you expected to see?"

- "Do you care to explore anything on this page?"

- "What would you do if I wasn't here? I'd like you to do whatever you'd normally do."

In Use Case 2, the participant was asked to perform a database query, and view and interact with the information presented on the Query Results page. We provided 
the participant with a list of four query criteria, and guided the participant using pre-determined, open-ended questions, adapted from Steve Krug (2009):

- "Let's pretend that you are interested in viewing all public Tweets that meet the following criteria:"

1. Include the phrase "ParaTranspo"

2. Include the hashtag "\#PWD"

3. Tweeted on "January 12021 "

4. Include a media attachment of the type: "Image"

- "What are you going to do first? What are you thinking?"

- "Is that what you expected to happen?"

- "What do you think about the query results? Was there something in particular that made you think that?"

- "Go ahead, explore a little bit, please select one of the icons on the map."

- "Is that what you expected to happen?"

- "What are you looking at? What are you thinking?"

To conclude the usability-testing session, the participant was asked if they had any questions about the prototype, or the study in general. We expressed our view that: "It is incredibly helpful to have your feedback before our team considers a software build. For one thing, it helps us better understand the potential for a product similar to the prototype we evaluated today. And second, we believe that the best software builds are completed hand-in-hand with our community partners from day one; this way, we don't waste your time asking you to test a product that you didn't ask for, and our time isn't wasted building a product that does not address your needs." Following the Q\&A, the participant was emailed a 10-question post-test questionnaire (SUS) and asked to return it via email, and provide contactless drop-off or a mailing instruction for receipt of the "Thank You" card enclosed with a \$20 Tim Horton's gift card, as compensation for participating in the study. 


\section{Stakeholder Group 2}

Participants who self-identified as a member of the public transit advocate/lobby community completed two use cases during the remote usability-testing session. Following evaluation of the use cases, the participant was offered an opportunity pose questions, and was provided with a post-test questionnaire, via email. After the participant returned the post-test questionnaire via email, they were asked to provide instruction for a contactless delivery, or provide a mailing address to facilitate delivery of a "Thank You" card enclosed with a $\$ 20$ Tim Horton's gift card, as compensation for their participation in the study.

In Use Case 1 (same as Stakeholder Group 1), the participant was asked to view and explore the functionality of the 'Landing' page. We guided the participant using pre-determined, open-ended questions, adapted from Steve Krug (2009).

In Use Case 2, the participant was asked to perform a database query (similar to Stakeholder Group 1, but query criteria included one additional item), and view and interact with the information presented on the Query Results page. We provided the participant with a list of five query criteria, and guided the participant using pre-determined, open-ended questions, adapted from Steve Krug (2009):

- "Let's pretend that you are interested in viewing all public Tweets that meet the following criteria:"

1. Include the phrase "ParaTranspo"

2. Include the hashtag "\#PWD"

3. Include the Twitter handle: "@OttTransitRider"

4. Tweeted on "January 12021 "

5. Include a media attachment of the type: "Image"

- "What are you going to do first? What are you thinking?"

- "Is that what you expected to happen?"

- "What do you think about the query results? Was there something in particular that made you think that?" 
- "Go ahead, explore a little bit, please select one of the icons on the map."

- "Is that what you expected to happen?"

- "What are you looking at? What are you thinking?"

We concluded the usability-testing session in the same manner as Stakeholder Group 1.

\section{Stakeholder Group 3}

Participants who self-identified as an elected and or appointed member of the Transit Commission completed two use cases during the remote usability-testing session. Following evaluation of the use cases, the participant was offered an opportunity pose questions, and was provided with a post-test questionnaire, via email. After the participant returned the post-test questionnaire via email, they were asked to provide instruction for a contactless delivery, or provide a mailing address to facilitate delivery of a "Thank You" card enclosed with a $\$ 20$ Tim Horton's gift card, as compensation for their participation in the study.

In Use Case 1, the participant was asked to view and explore the functionality of the 'Landing' page. We guided the participant using pre-determined, open-ended questions, adapted from Steve Krug (2009).

In Use Case 2, the participant was asked to view the 'Dashboard' page, and select a 'Tweet Story' published by a community partner, to examine in greater detail. The participant was presented with a 'Tweet Story', visualized in a mock cybercartographic atlas and adjacent information timeline. We guided the participant using pre-determined, open-ended questions, adapted from Steve Krug (2009), such as:

- "Please select a tweet story, curated by one of our community partners, to explore in greater detail"

- "What are you looking at?"

- "What are you thinking?" 
- "Is this what you expected to see?"

- "Is this information useful to a public transit decision-maker?"

- What is missing? What can we do to improve this page so that it may become a useful tool for public transit decision-making?

To conclude the usability-testing session, a participant was asked if they had any questions about the prototype, or the study in general. We expressed our view

that: "It is incredibly helpful to have your feedback before our team considers a software build. For one thing, it helps us better understand the potential market for a product similar to the prototype we evaluated today. And second, we believe that the best software builds are completed hand-in-hand with our community partners from day one; this way, we don't waste your time asking you to test a product that you didn't ask for, and our time isn't wasted building a product that does not address your needs." Following the Q\&A, the participant was emailed a 10-question posttest questionnaire (i.e., System Usability Scale), and asked to return it via email, and provide contactless drop-off or a mailing instruction for receipt of the "Thank You" card enclosed with a \$20 Tim Horton's gift card, as compensation for their participation in the study.

\section{Challenges}

A few significant challenges exist when designing remote usability-testing sessions. We consider these challenges in two groups:

1. Items we have some degree of control over; and,

2. Items we do not control.

Our focus when designing remote usability-testing sessions, must remain on items that we have some degree of control over. These items include: robustness of prototype design, designing for multiple interaction styles and interaction mediums, and the inclusion (or exclusion) remote graphical content that can render the prototype resource intensive (and inoperable on technological devices with limited RAM and 
or CPU power). Items we do not control include: a participant's technological device and operational skill to configure the remote usability-testing session on their native device; access to high-speed broadband internet, and customization of internet browser settings and applicable plug-ins. We have described some of the steps we took to make our prototype as accessible as possible, across as many technological mediums as possible, for participants with varying degrees of technological skill level. We describe some of the challenges that arose during the remote usability-testing session in chapter 4 . 


\section{Chapter 4}

\section{Results}

\subsection{Field Observations}

We passively observed the public transit conversation at Town Halls, City Council and Transit Commission meetings, visited major transit hubs during peak service hours, and observed the public transit conversation happening on Twitter. The purpose of the field observations was twofold: first, we hoped to learn about issues of importance to public transit users in Ottawa; and second, we hoped to document any meaningful difference in terms of what and how public transit issues are discussed in different formal and informal environments. As we conducted our field observations, we also noted significant barriers to access the public transit decision-making process, especially among members of the PWD community.

In November 2019, we passively observed a Public Transit Town Hall in Centretown. The meeting was organized and moderated by Member of Provincial Parliament (MPP) and "Official Opposition Critic for Seniors, Accessibility and Persons with Disabilities", Joel Harden. Topics of conversation included: the state of the public transit system, and detailed discussion about the launch of the 2.1B LRT the Confederation Line. We also observed discussion about the definition about the definition of an 'accessible' public transit station.

The Town Hall meeting was structured as an organized panel discussion. Panellists

included: Sally Thomas (PWD community), Shawn Menard (Ottawa City Councillor) and Sarah Wright-Gilbert (Citizen Transit Commissioner), and the discussion was moderated by Joel Harden (MPP Ottawa Centre). Topics of discussion included:

1. What does it mean for public transit to be accessible?;

2. Increased bus service to hospital campuses;

3. Better communication practices from the public transit authority; and, 
4. Climate focused solutions to public transit problems [48].

What did the panellists have to say? Menard was quite articulate: "Our riders are the ones that know the system best, we need to listen to them" [48]. Menard spoke to the fact that Ottawa's public transit system is not successful, citing bad planning and a broken procurement process that lacks any real accountability to the public, who ultimately pay for the system with their tax dollars. Moreover, Thomas highlighted the lack of accessible public transit infrastructure around the city, and reminded attendees that in order to have a world class transit system, the system must be accessible and inclusive to those with visible and invisible disabilities. Thomas described her experience using the public transit system, including the new LRT system: "We need to have more cooperative seating on buses, because many people of many abilities use those seats. When I travel on the LRT, my chair takes up two seats and there are only four accessible seats on a car, so I usually have to be separated from my friends." [48]. Wright-Gilbert, both a transit user and citizen member appointed to the Transit Commission, noted the need for more transparency and accountability from the local transit authority about the inner working of the public transit system. Wright-Gilbert also reminded attendees that the public transit system must serve the communities' needs by ensuring that buses and trains are not overcrowded, so that they remain accessible to everyone [48].

At the conclusion of the Town Hall, Joel Harden advised: "Now that we have heard your stories, we want to do our part to amplify them. We have, and will continue to bring your stories to the Ontario Government and [local transit authority], via Councillor Shawn Menard's office. We also know that we need a new deal for environmental policies in Ontario. The status quo will no longer work and what we heard at this town hall will help us shift that conversation so we can continue to fight for a liveable planet" [48].

We passively participated in a second Town Hall in Old Ottawa South, organized and moderated by Ottawa City Councillor, Shawn Menard. The conversation centred around the reliability of two key bus routes that serve Capital Ward (including the Bank Street Business Improvement Area (BIA) and Carleton University). The City of Ottawa provided access to two staffers (i.e., city engineers), and discussed possible 
solutions to improve the reliability and accessibility of the bus system. We noted delegations from the public transit advocate/lobby, the Glebe BIA, school board trustees, and local residents. We passively observed the discussions that took place with the intent to document the conversation, and organize it such that it could be made accessible to the public transit decision-making body, the Transit Commission.

In November and December 2019, we passively observed three Transit Commission meetings at Ottawa City Hall. We observed the public transit authority's senior management team and elected and appointed members of the Transit Commission discuss and vote on the 2020 Draft Operating and Capital Budget, in addition to several other items related to the planning and operation of the public transit system. Public delegates, citizens and media also attended the meetings and sat in the gallery. A non-exhaustive list of items that were discussed and are related to our work include:

1. The EquiPass and Community Pass (i.e., a subsidized bus pass for low income residents, including members of the PWD community) and discussion of a potential bus fare freeze;

2. Public engagement strategies;

3. The need for improved communications and wayfinding at major transit hubs;

4. ParaTranspo (i.e., accessible transit for PWD) service enhancements (e.g., an online booking system, increased hours of service, etc.);

5. Understanding demand for ParaTranspo and costs associated with purchasing additional fleet vehicles to meet current demand; and,

6. Platform overcrowding and major transit station upgrades (e.g., platform expansion and redesign) $[24,25,26]$.

The Transit Commission heard from the following public delegations:

1. A member of the PWD community spoke about the need for firm dates and deadlines, greater transparency, and noted that the online booking system be available 24/7/365, and additionally that ParaTranspo buses be equipped with 
the Presto Card system (i.e., an electronic payment method currently available to conventional transit users);

2. A member of the public transit advocate/lobby asked for increased opportunity to participate in the decision-making process regarding the implementation of the ParaTranspo online booking system. They advised "that the users' group be comprised of diverse users who could provide better feedback";

3. A member of the public transit advocate/lobby community asked for an increase in the number of ParaTranspo buses in addition to the implementation of an online booking system, and noted that "users were currently being denied rides because of a lack of buses"; and,

4. A member of the Healthy Transportation Coalition (i.e., public transit advocate/lobby community) "asked for the application of an equity and inclusion lens in the planning of the online booking system" $[24,26,26]$.

Our observation of the public transit discussion at Ottawa City Hall highlighted an opportunity to grow citizen participation in the public transit decision-making process. We noted the meeting structure, and the capacity for public delegation to proceed with digital presentations, provided the five-minute presentation allotment was respected. We consider public delegation at the Transit Commission an optimal environment to deploy cybercartographic atlases that highlight existing public transit issues, and provide informative narratives, constructed with actual public sentiment retrieved via the API economy and expressed in language that is actionable (i.e., in the form of a motion to be debated, or as a directive to the public transit authority).

In our observation of major public transit hubs during peak service hours, we noted several accessibility and wayfinding challenges. The East and West LRT terminus stations presented the most significant challenges. At Blair Station (i.e., East LRT terminus), we observed significant platform overcrowding and a lack of wayfinding information [119]. Moreover, the service elevator that connects the transit station with a multi-use pathway to a nearby shopping centre was not in service at the time of our visit. As a result, transit users who disembarked at Blair Station were required to take an alternate route to access shopping centre. We did not observe 
any wayfinding instructions that described alternate routes to the shopping centre in the event that the elevator ceased to operate. We also observed several public transit staffers stationed around the terminus stations during peak service hours. The staffers were tasked with providing wayfinding and accessibility information to transit users during peak service hours, but were not available to assist transit users outside of peak service hours [33].

We observed similar accessibility and wayfinding issues at Tunney's Pasture Station (i.e., West LRT terminus). After disembarking from the LRT, public transit users were required to travel over 250 meters from the train platforms to the nearest bus platform. Travel times from the train platforms to the bus platforms for an able-bodied person exceeded five minutes. During peak service hours, travel times from the train platforms to the bus platforms increased significantly due to platform overcrowding. We observed limited platform space, a lack of wayfinding information, and an abundance of public transit users loitering on the platforms as they waited for a connections. Inclement weather further compounded loitering behaviour, and snow removal was a challenge due to overcrowding and limited platform space.

While we acknowledge that the existing LRT terminus stations will lose their distinction as 'terminus stations' following the next phase of LRT expansion (to be completed in 2026), the platform and station designs we observed at the time left much to be desired. Our initial observations were later confirmed by the local transit authority at the Transit Commission meeting, as social media depictions of station conditions, including mass confusion, transit users jumping over fences, overcrowding, etc. led the Transit Commission to issue a mandate to the public transit authority to consider complete station redesigns, including expansion of both the east and west terminus bus platforms [119]. Following several weeks of strenuous commuting conditions for public transit users, the Transit Commission directed the public transit authority to redesign and expand the East and West terminus bus platforms to better accommodate transit users' needs [79]. The Transit Commission cited several dozen social media posts during monthly meetings, and multiple Ottawa City Councillors who were not formal members of the Transit Commission often participated in the 
meetings as passive participants, armed with public transit user insights, communicated via Twitter, and in some instances, Facebook.

The use of social media to convey public transit narratives directly to public transit decision-makers contributes to the democratization of public transit decision-making. Our observations at Ottawa City Hall, during Transit Commission and City Council meetings, highlighted the existing role of social media in municipal decision-making. Further, the Mayor of the City of Ottawa has publicly called on City Councillors to maintain "a high level of accessibility through social media" [36]. We chose to observe the public transit conversation happening on Twitter for three reasons:

1. $92 \%$ (12/13 members) of the Transit Commission maintain a level of public accessibility via the social media platform, Twitter;

2. Members of Transit Commission routinely introduce questions and motions based on interactions that originated on the social media platform; and,

3. From a research perspective, the Twitter API is accessible and highly customizable, used by politicians, journalists, and academics around the world, and can provide access to rich datasets, including text-based tweets and multimedia content, without financial considerations* (a fee structure does exist for unrestricted data collection, but for the purposes of this work, we do not anticipate any financial costs to access Twitter data. This is in part due to the robust cache capacity built into the Nunaliit Cybercartographic Atlas Framework.)

\subsection{Semi-Structured Interviews}

We curated three versions of the semi-structured interview - one for each public transit stakeholder group. Each semi-structured interview contained three sections:

1. A brief introduction (participants were encouraged to self-identify any physical disabilities or additional barriers they may encounter while using public transit infrastructure, as well as describe a hobby or job - where applicable);

2. Understanding a participant's experience using public transit infrastructure (both physical and digital infrastructure); and, 
3. Understanding a participant's experience using the social media platform, Twitter, to converse with others about public transit issues.

\subsubsection{General Findings for Stakeholder Group 1}

We spoke with three participants who self-identified as living with a disability or experiencing additional barriers to access public transit infrastructure, and or the public transit decision-making process. Participants were asked to speak candidly about their recent experience using the public transit system, and additionally, to describe their interactions with public transit decision-makers in both formal and informal venues. Participants were then asked to describe their use of the social media platform, Twitter, to participate in the public transit conversation happening on the platform. Participants reported a mean age of 40 years, and all participants identified as male.

All participants reported using a mobility device in their day-to-day activities. While day-to-day activities varied for each participant, all participants reported using the public transit system (or an accessible taxi service contracted by the public transit authority) in their day-to-day activities. All participants identified as actively employed and noted accessible transportation needs directly related to their employment. All participants expressed a strong desire to challenge the status quo for what constitutes an accessible public transit system.

Participants were asked to describe their use of the public transit system, including use of conventional (bus and LRT) and or paratransit service. The local public transit authority deploys several accessibility features in their bus and train fleets that are intended to provide an accessible transportation option to a wide range of users with varying accessibility needs. Some of the core accessibility features in the conventional bus and train fleet include:

1. 'Kneeling' feature lowers the front door to curb level for easier boarding;

2. Slip-resistant ramp with raised edges to prevent an assistive device (e.g., wheelchair, walker or scooter) from rolling off the side; 
3. Cooperative (i.e., priority) seating near the front of the bus for users who have difficulty standing on a moving vehicle;

4. Automatic announcement of next stops over the speaker system inside the bus: Next Stop Announcement System (NSAS);

5. Exterior auditory announcements of the route and destination of the arriving bus;

6. Visual display of the next stop, bus route, destination and time on a digital signboard inside the bus;

7. Warning light and audible alarm when the bus or ramp is raised or lowered;

8. Slip-resistant, low-glare floors;

9. Lights at the entrances and inside the bus to help users board and alight; and,

10. High-contrast handholds and grab bars [80].

The paratransit bus fleet provides an additional level of accessibility, relative to conventional service, with available operator assistance for boarding and alighting, as well as access to a heavy duty ramp to accommodate heavy self-propelled mobility devices [80]. Paratransit bus service is an on-demand service (i.e., users are required to confirm a trip $24 \mathrm{~h}$ in advance). Participants described the process of booking a paratransit trip as an arduous process that often requires the user to call a dispatcher and remain on hold for fifteen minutes to one hour, as demand for the service far outpaces available resources. The public transit authority recently launched an online trip booking system. Early user reviews of the online booking system are positive; however, several bugs remain unresolved as the system is in its infancy. Participants described difficulty accessing the online booking system using a mobile/tablet device, and noted various functions that were not yet available, such as booking a service animal accommodation. Participants also described challenges with on-time performance and fare payment, and expressed frustration with the subcontracted accessible taxi service, describing it as "unreliable" and "does not meet the operational standards set by the public transit authority". 
All public transit systems experience on-time performance issues, but these issues are compounded for members of the PWD community who rely on paratransit service exclusively for their day-to-day activities. Paratransit boarding and alighting times are scheduled in 30-minute windows. For example, if a paratransit user requests a pick up at 9am, they must wait at the pick up location for thirty minutes before they are allowed to contact the dispatcher and inquire about the status of their bus. If the pick up location is outdoors, unfavourable weather conditions such as heavy precipitation and or extreme temperatures can make the trip unbearable for some. Participants reported lengthy delays during AM and PM peak service hours, as well as significant delays during adverse winter driving conditions. Participants also noted discrepancies in hours of operation and available payment methods for paratransit service, relative to conventional service.

The public transit authority currently operates twenty-four-hour conventional bus service to key amenities located throughout the city, but does not offer paratransit users twenty-four-hour service to those same amenities. Users who rely exclusively on paratransit service expressed frustration that conventional transit users can access major universities, hospitals and airports at all hours of the day, while paratransit users are restricted to accessing those same amenities during reduced operating hours (e.g., 5:00-24:00h). Further, current City of Ottawa By-laws permit ride sharing companies to pay an additional $\$ 0.10$ 'accessibility fee' per ride, in lieu of providing actual accessible vehicles for hire in the city. "In 2019, Council approved a strategy to use the funds from the voluntary surcharge [accessibility fee] for programs supporting accessible transportation."; however, members of the PWD community that we spoke with described a large accessibility gap that continues to exist despite the additional funds marked for "programs supporting accessible transportation" [89].

Participants were asked to describe the five most urgent issues facing the PWD community related to public transit: an overarching theme of 'equitable service' emerged. This was evidenced in participant recollection of their experiences using conventional service vs. paratransit service. Participants described many instances where the needs of conventional transit users were privileged over paratransit users. 
For example, participants described multiple instances in which they felt discriminated against because of their disability, or some additional barriers to access they experienced while using the public transit system. While using paratransit service, participants described inequitable hours of service, payment methods and operating standards, relative to conventional transit. When queried about barriers to access the public transit decision-making process, participants expressed an optimism to drive policy-level change using social media channels, and cited productive interactions with public transit decision-makers, via Twitter. We asked participants about their social media interactions with public transit decision-makers on Twitter, and quickly recognized an opportunity to access a rich dataset that describes the existing inequity in the public transit system with images, audio/visual media and text.

Although all participants indicated active employment and receipt of financial support from the Ontario Disability Support Program (ODSP), they self-reported as living below the poverty line $(\$ 25,153$ [101]) in the province of Ontario. With limited discretionary income, participants expressed an inability to pay for travel to and from Ottawa City Hall in order to participate in the public transit decision-making process. Instead, participants described an alternate route to access the public transit decisionmaking process - through social media information campaigns, often amplified by non-PWD community members with political capital, significant financial resources, and or by the public transit advocate/lobby community.

Participants recalled successful lobbying outcomes when they voiced their accessibility concerns directly to local media, via Twitter. Local media helped to amplify individual and community narratives that highlighted some of the challenges facing the PWD community. Twitter was noted as a preferred medium for conveying personal narratives using text and audio/video media, because members of the Transit Commission maintain a high degree of public visibility on Twitter. Participants recalled multiple instances in which a tweet that originally tagged a media organization went on to be discussed at Transit Commission, often following significant media amplification. Participants also reported that some members of the Transit Commission respond directly to their tweets and are willing to raise their concerns at the monthly Transit Commission meeting, but these instances represent an exception to the norm. 
In one instance, a candidate for an upcoming municipal election included the implementation of a new accessible curb cut as part of an election platform. The candidate was successful in their election bid, and the accessible curb cut was implemented, thereby fulfilling a campaign promise and ending a multi-year campaign to render the bus stop in question accessible to people who use a mobility device.

Participants were asked to describe their participation in the accessible public transit conversation happening on the social media platform, Twitter. Specifically, participants were asked to indicate 'hashtags' and 'handles' active in the public transit conversation happening on the platform. Hashtags and handles are important markers of what is being discussed, and by whom. We compiled participant responses, as this information is critical to building high-quality data filters using the Twitter API. The most active hashtags in the accessible public transit conversation include:

1. \#PWD

2. \#ParaParity

3. \#ODSPoverty

4. \#ParaTranspo

5. \#OCTranspo

6. \#Accessibility

7. \#Equity

8. \#Infrastructure

The most active accounts (excluding participant Twitter accounts) in the public transit conversation include multiple Ottawa City Councillors, local journalists, and public transit advocates:

1. @CMkenney

2. @ShawnMenard1 
3. @HorizonOttawa

4. @OttTransitRider

5. @HealthTransport

6. @sallythomas1

7. @JonathanWilling

8. @jchianello

9. @KatePorterCBC

10. @tm_kavanaugh

11. @RiverWardRiley

12. @TonyCarricato

13. @TKurdi

In summary, our conversations with members of the PWD community allowed us to collect and organize sets of keywords, hashtags, and handles that are active in the accessible public transit conversation happening on Twitter. This information is critical to building high-quality data filters using the Twitter API. A highquality data filter can help establish understanding, discover new insights, identify trends, and highlight new relationships among data. Further, the implementation of a high-quality data filter can provide access to a rich set of high-quality images and audio/video media that depict the day-to-day experiences of the PWD community, while they use the public transit system. The resulting high-quality dataset can then be used to populate a cybercartographic atlas in which the data can be organized and presented to multiple stakeholders with varying mandates, while maintaining temporal-spatial attributes, where appropriate. 


\subsubsection{General Findings for Stakeholder Group 2}

We spoke with two participants who self-identified as active members of the public transit advocate/lobby community. Participants were asked to speak candidly about their recent experience using the public transit system, and additionally, to describe their interactions with public transit users and public transit decision-makers, in both formal and informal venues. Participants were then asked to describe their use of the social media platform, Twitter, to participate in the public transit conversation happening on the platform. Participants reported a mean age of 41 years, and both participants identified as male.

Participants reported limited recent experience using conventional bus service (bus and LRT) in their day-to-day activities, and no experience using paratransit service (either directly as a user, or indirectly as a companion). Participants reported a strong desire to use the public transit system more frequently, but cited employment demands as a reason why they were unable to do so. Participants noted immediate family, namely spouses and children, as frequent public transit users. Neither participant self-identified as living with a disability, or facing additional barriers to access the public transit system, or the public transit decision-making process. We deliberately sought to speak with these two individuals after we noticed their contributions to public transit decision-making, in both formal and informal venues, as well as their presence in the public transit conversation happening on the Twitter.

During our field observations, we observed participants actively taking part in the accessible public transit conversation in formal and informal environments. Notably, we observed participants using their professional skill sets to advocate and lobby public transit decision-makers on behalf of members of the PWD community who rely on accessible public transit infrastructure in their day-to-day activities. One participant is a founding board member of a local public transit advocacy group, holds a $\mathrm{PhD}$ in American History and Political History, and has extensive experience interacting with elected officials and running political campaigns. The second participant is a local architect, and member of the Ontario Association of Architects (OAA), Architecture Canada (RAIC), and the American Institute of Architects, in addition to serving as 
the president of a local architecture firm. We note the specific skill sets of the participants because they are relevant to our goal of helping to shape the public transit conversation in order to improve policymaking outcomes.

Participants expressed a strong desire to use Twitter to raise awareness and engage in conversations about accessible public transit. Participants reported using their platform (i.e., social media reach) to convey messages about inclusive environments and accessible communities, forged through accessible, multimodal public transit systems. They described their role as an accessible public transit advocate as speaking on behalf of marginalized groups who are often under-represented in the public transit decision-making process. One participant described their twitter advocacy as: "For me, being able to use a medium like Twitter, where I can call attention to an issue, and then bring awareness of not just how to make things more accessible to the person using a wheelchair who can't get over a snow bank, but also how that affects parents with strollers, little kids, the elderly, and then how that leads to a bigger conversation about the type of city we want to have as a whole". Further, when we inquired about the inclusion of media attachments in social media posts, for example, images and audio/video media in addition to text-based tweets, and its impact on the reach of a tweet (i.e., how many user interactions it receives), participants described several instances in which they believed that additional media attachments were instrumental in conveying their message to a wider audience.

Participants described the process of including images in tweets as a mechanism to convey an idea with context, when character limits are a constraint. Participants described Twitter's 280-character tweet limit as an opportunity to reference specific hashtags and tag an audience, in order to increase the reach of a tweet. Participants also described the inclusion of images and audio/video media in tweets as a practice that increases reach, without compromising understanding. The adage: "A picture is worth a thousand words", reigns true in the Twittersphere, where still images are used to communicate complex ideas and convey meaning or essence more effectively than mere verbal descriptions alone.

Participants also described using hashtags that are accessible to people who use 
screen readers in their social media advocacy. For example, "\#accessibilitymatterstome" is not accessible to an individual who uses a screen reader, whereas "\#AccessibilityMattersToMe" is; this is known as CamelCase, and is defined as the practice of writing phrases without spaces or punctuation, indicating the separation of words with a single capitalized letter, and the first word starting with either case. The implementation of accessibility hacks in social media advocacy for accessible public transit infrastructure can be easily overlooked by advocates who are not familiar with digital accessibility tools such as screen readers, thus the communities that are the intended beneficiaries of the accessibility advocacy, may struggle to recognize the advocacy efforts at all.

Participants also noted a lack of accessible restrooms at major transit hubs as a barrier to access for many residents, including members of the PWD community. Participants referenced situations where people of all ages and abilities, including families with young children, were unable to use the public transit system due to a lack of robust restroom amenities. They described the lack of public restroom amenities as "conscious choices by decision-makers that lead to inequitable access of public spaces", and cited a broken infrastructural procurement process as a major contributor to the problem of inequitable public spaces. The resounding sentiment from participants was that public transit decision-makers continue to procure infrastructure that provides the best value for money (i.e., value=price) for the tax payer, but rarely consider the social capital of our built environment, including the notion of equitable public spaces, including an equitable public transit system. Participants noted that we will continue to experience inequity in our build environments so long as we continue to equate the value of our public transit system with the price of the assets that underpin it.

Participants also expressed accessible public transit advocacy work in range of written publications including: personal blogs, and local and national newspapers editorials. During our field observations, we noted several written publications that were additionally communicated as tweet attachments in the public transit conversation happening on Twitter. Participants described hyperlinked attachments as another mechanism to communicate complex ideas, while using the 280-character tweet 
limit to tag a specific audience and attract attention, and entice users to interact with the hyperlinked item. We observed public transit decision-makers referencing tweet attachments during Transit Commission meetings, and additionally forwarding those attachments in direct communication with the public transit authority.

In summary, our conversations with members of the public transit advocate/lobby allowed us to improve our understanding of the role the public transit advocate/lobby community plays as an intermediary between members of PWD community and public transit decision-makers. We noted the methods and media of communication, and used that information to inform the design of the public transit advocate/lobby community user profile in the prototype design. We implemented additional buttons in the public transit advocate/lobby user profile, relative to the PWD community profile, to reflect needs identified during the semi-structured interview. Notably, we added three unique functions to the Public Transit Advocate/Lobby user profile:

1. (Button) Add Related Document (i.e., enables uploads of local documents to an existing tweet story, e.g., a text file, an image, an audio/video file, etc.);

2. (Button) Share Tweet Story (i.e., creates a shareable link that can be pushed laterally to another community group/NGO, or to a recognized media organization); and,

3. (Button) Publish for Transit Commission (i.e., creates a shareable link that can be pushed directly to all members of Transit Commission with a single click).

\subsubsection{General Findings for Stakeholder Group 3}

We spoke with a single participant who self-identified as a member of the Transit Commission. The participant was asked to speak candidly about their recent experience using the public transit system, and additionally, to describe their interactions with members of the PWD community and public transit advocate/lobby community, in both formal and informal environments. The participant was then asked to describe their use of the social media platform, Twitter, to participate in the public transit conversation happening on the platform. The participant reported an approximate age of 39 years, and identified as female. We tentatively scheduled semi-structured 
interviews with two additional members of the Transit Commission, but were ultimately unsuccessful in conducting the interview. The prospective participants cited urgent, unplanned employment demands, and offered to reschedule at a later date, if possible.

The participant reported extensive use of the conventional transit service in their day-to-day activities, and no experience using paratransit service (either directly as a user, or indirectly as a companion). The participant reported using conventional bus service and LRT during AM and PM peak service hours in their employment commute. When queried about non-employment commutes, the participant indicated a preference to use their personal vehicle or hire a ride hailing service, and cited increased personal convenience.

The participant expressed a strong desire to use Twitter to raise awareness and engage in conversations about accessible public transit. The participant referenced multiple social media posts that were intended to spark discussion about issues related to accessible public transit on the social media platform, that subsequently appeared on local news broadcasts, at times in the form of a public opinion pole or social media segment. The participant also emphasized that many non-Twitter users are exposed to some of the conversations happening on the platform through third party entities such as media organizations and broadcast news, or other social media platforms that allow cross-platform content sharing. When queried about the limitations of conveying information in 280-characters or less, the participant reported using tweet threads (i.e., a series of connected tweets) as a mechanism to convey complex ideas and circumvent the 280-character tweet limit.

The participant reported using their mobile phone and laptop computer to participate in the accessible public transit conversation happening on Twitter. They described using their mobile phone to live tweet during commutes, and reported using their laptop computer to tweet during work hours. The participant also reported livetweeting during Transit Commission meetings to increase accessibility to the public transit decision-making process for citizens who were not able to attend the meeting in person, or virtually. The participant described using the screen capture function to publish sections of word documents, charts, and infographics that were initially 
introduced at the Transit Commission, to a wider audience on Twitter.

The participant described accessible public transit as: "transit that is more convenient than hopping in the car and driving where you need to go", but also acknowledged that the term 'accessible public transit' carries a different definition for people who rely on public transit for all of their day-to-day activities and do not have access to personal vehicle. The participant described accessible public transit for this second group as "must be reliable and affordable, and be more convenient than hiring a taxi". During the annual public transit budget vote (at Transit Commission), the participant reported posting pre-defined remarks about various budget items on Twitter, with the hope of sparking a larger conversation about transit fares and accessibility of the public transit system for low-income communities.

When asked to elaborate on their use of screen captures as tweet attachments, the participant stated that their goal was to communicate effectively: "I didn't want to parse it or shorten it, just to put it into a tweet" (referring to tweets that contained screen captures of information contained in internal memos that were not considered confidential). The participant expressed a desire communicate transit news (e.g., service changes) in a timely and accessible manner, by targeting a specific audience using hashtags such as '\#OttNews' and '\#OttPoli'. They also described the use of hyperlinks to drive tweet engagement and provide additional resources for Twitter users who require supplemental public transit information.

When asked to describe the process of debating a motion at Transit Commission, the participant described preparing questions and remarks prior to the start of the meeting, as well as taking notes during the meeting. Prepared questions often included references to social media posts, but the participant reported that "social media posts are not taken as seriously as say, an official complaint registered with [the public transit authority] directly". The participant expressed concern that some tweets can be perceived as "an exaggeration" of actual events that transpired, and cited the use of social media in formal motions as a "double-edged sword". The participant did recall collecting information from social media and forwarding that information directly to other members of the Transit Commission, or directly to the public transit authority, but did not recall using information gathered from social 
media for the explicit purpose of introducing or debating a formal motion.

We asked the participant to describe any challenges they faced in terms of collecting, organizing and storing social media information to be introduced as a testamentary instrument, for example, at the Transit Commission. The participant reported being tagged in tweets at all hours of the day, being unable to answer many queries, and at times, being overwhelmed by the volume of information directed at them. The participant also recalled directing multiple Twitter users to the public transit authority's online comment/complaint form (i.e., via hyperlink), and directing other Twitter users to contact their local City Councillor where appropriate. If the com-

plainant expressed discomfort or an inability to forward their complaint to another party directly, the complainant was asked to communicate their issue in a formal email to the transit commissioner, that could in turn be forwarded to the appropriate authority.

In summary, our conversation with the member of the Transit Commission highlighted significant challenges associated with learning about community needs related to accessible public transit, via Twitter. The participant reported an inability collect and organize public sentiment originating on Twitter, in order to conduct needs assessments for the various communities that use the public transit system in Ottawa. Further, the participant defined the practice of using social media posts as a testamentary instrument in official decision-making activities as a double-edged sword, due to the "exaggerated nature of social media content".

\subsubsection{Summary}

The semi-structured interviews were used to establish a mandate for three public transit stakeholder groups in Ottawa. The semi-structured interview was designed to answer two core research questions, and additionally served to define the existing public transit decision-making IS:

1. What are the needs of the PWD community in terms of access to public transit infrastructure and access to the public transit decision-making process? What barriers to access exist, and what can be done to reduce the number of barriers? 
2. How must the existing public transit IS change in order to incentivize community narratives over intuitive judgements, at the policymaking level?

The information gathered during the semi-structured interviews was compiled for each stakeholder group and used to define the existing public transit decision-making IS in Ottawa. We were able to map the bidirectional flow of information between the public transit user, the public transit advocate/lobby, and to the public transit decision-making body, the Transit Commission. We discuss the existing IS as it relates to the public transit decision-making process, and propose a novel IS predicated on the social capital created in the process of Cybercartography, in chapter 5 . We implement the findings of the semi-structured interview in the design of a low-fidelity prototype that features a mock cybercartographic atlas, as the central artefact. We evaluate the prototype with a database query and results exploration task during a remote usability-testing session, with three public transit stakeholder groups. At the conclusion of the usability-testing session, participants completed a 10-question posttest questionnaire (i.e., System Usability Scale). We report the results of the remote usability-testing sessions, including the post-test questionnaire, in the next section.

\subsection{Remote Usability-Testing Sessions}

\subsubsection{General Findings for Stakeholder Group 1}

We conducted a remote usability-testing session with three participants who selfidentified as living with a disability and or experiencing additional barriers to access public transit infrastructure, and or the public transit decision-making process. Participants viewed and explored a Landing page, and performed a database query task and results exploration task. Two use cases were evaluated during the remote usability-testing session:

1. Use Case 1: View and explore the functionality of the Landing page, and select an appropriate user profile and navigate to the Database Query page; and,

2. Use Case 2: Perform a database query by entering four unique query criteria into the Database Query page, and explore the query results, visualized in a 
mock cybercartographic atlas and adjacent information timeline, on the Query Results page.

Following evaluation of the use cases, participants were encouraged to speak candidly about their experience participating in the remote usability-testing session, and were provided an opportunity to pose questions. At the conclusion of the remote usability-testing session, participants were asked to complete a 10-question posttest questionnaire (i.e., System Usability Scale), via email. Following completion of the post-test questionnaire, participants provided contactless delivery information, or specified a mailing address for receipt of the \$20 Tim Horton's gift card, as compensation for participating in the study.

In Use Case 1, participants viewed and explored the Landing page, Figure 4.1. All participants correctly identified the presence of a GIS style map, with a public transit infrastructure layer that indicated the precise geographic location of public transit infrastructure in Ottawa. One participant initially described the public transit infrastructure layer as "important landmarks", and contrasted the GIS style map with Google Maps: "I'm trying to figure out how this is different from google maps". When prompted to interact with the Landing page, including the map, all participants attempted to select the '+' '-' icon (zoom function), embedded in the map. Participants expressed surprise when the zoom icon did not respond as expected. The map, including the public transit infrastructure layer and embedded zoom icon, was in fact a still image. After verbally describing the contents of the GIS style map and exploring its limited functionality, all participants selected an appropriate user profile and navigated to the Database Query page. 


\section{Home}

Welcome to The Accessible Public Transit Infrastructure Atlas! Our goal is to collect, organise and present the current conversation about accessible public transit infrastructure in a manner that serves to increase public understanding, and empower advocacy groups and public transit decision makers to build a public transit system that is accessible to everyone.

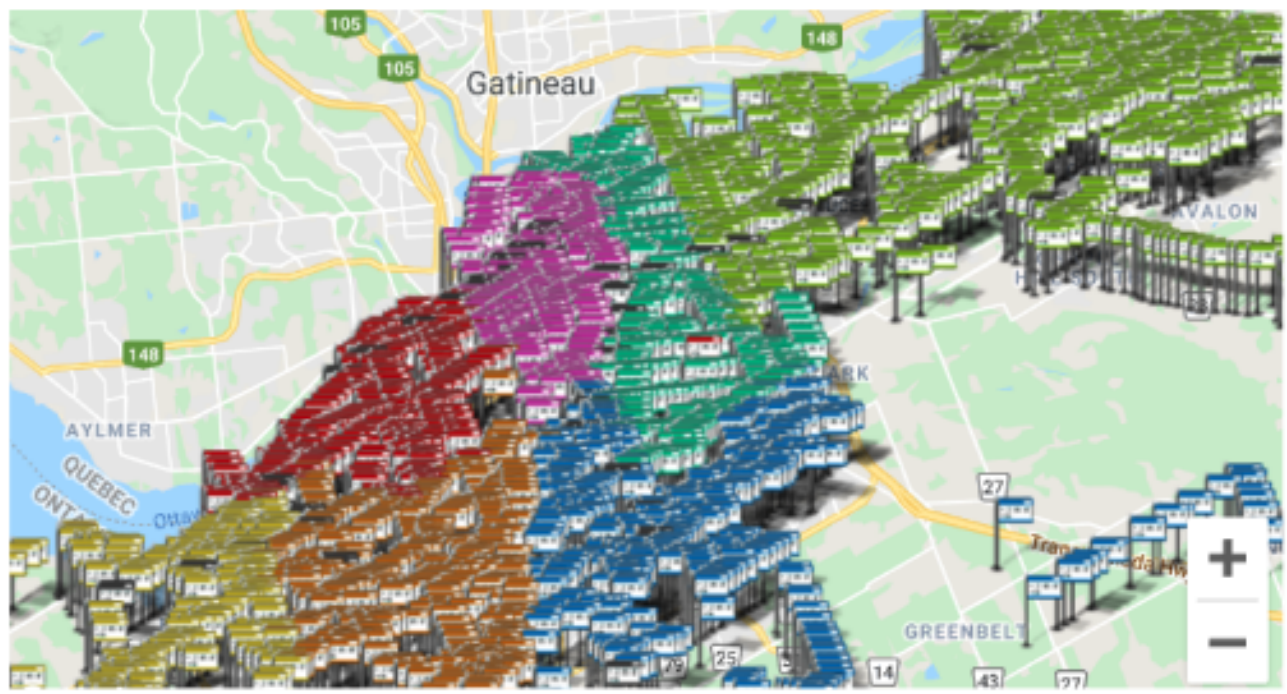

Which stakeholder lens do you want to use today?

Whatictict Public Transit User

Accessible Public Transit Advocate

Public Transit Decision Maker

Figure 4.1: Landing Page 
Participants described their internal thought process as they considered the three user profile options. Multiple participants expressed a degree of uncertainty when contemplating which user profile to select. Some participants viewed themselves as both a public transit user and a public transit advocate. All three participants ultimately selected the Public Transit User profile, and proceeded to the Database Query page.

In Use Case 2, participants performed a database query task by entering four predetermined query criteria into the Database Query page. Two of three participants made at least two significant errors while inputting query criteria (i.e., attempted to enter data in the wrong location), and one participant made a single error while inputting the four query criteria. All participants expressed a degree of surprise when they attempted to use the pop-up keyboard to input an initial query criteria. We reminded participants that the purpose of the session was to evaluate the user interface and not a participant's typing skill, hence, we autocompleted the query term for the participant once they had selected the appropriate input bar and selected the first letter of the query term using the on-screen keyboard. After a participant successfully entered all four query criteria in the appropriate bars, they selected the Search button and navigated to the Query Results page. Prior to selecting the Search button, we paused the participant and asked them to describe the content they expected to see on the Query Results page. The completed Public Transit User profile Database Query page is shown in Figure 4.2. 


\section{Home > Public transit user}

Welcome to The Accessible Public Transit Infrastruture Atlas--PUBLIC TRANSIT USER view! The information presented in this atlas was harvested from the social media platform, Twitter, and is plotted spatio-temporally on a GIS style map. The map is interactive by design, and includes audio-visual media, hyperlinks, URLs, and up to 280 characters of text. You may interact with content by selecting an icon on the map or by scrolling the tweet timeline. We are actively working to geolocate as many tweets as posssible.

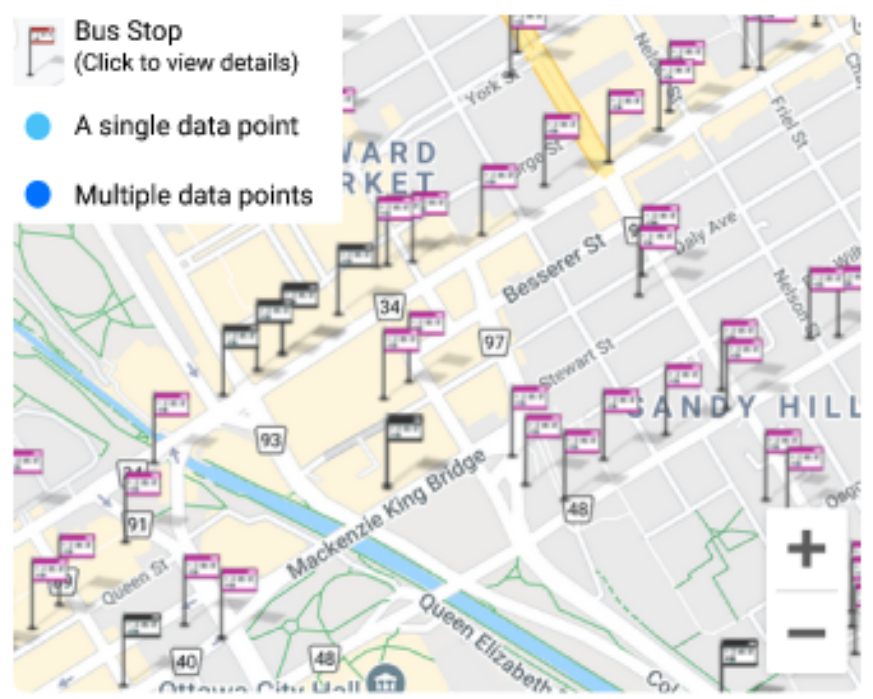

Find tweets with...

all of these words
none of these words
all of these hashtags
all of these accounts

Then narrow your results with...

date range

media attachments

location

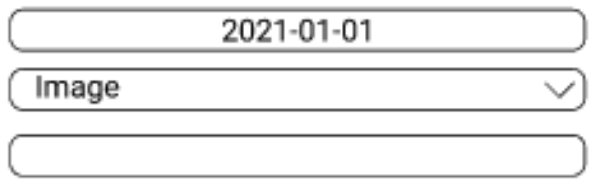

e.g. slect all that apply e.g. YYYY-MM-DD

e.g. 3052 or Parliament

To do this in the search box.

e.g. word1, word2, word3

e.g. -word1, -word 2

e.g. \#PWD, \#ParaTranspo

e.g. @CTVnews, @CBC

To do this in the search box.

\section{Search}

Figure 4.2: Stakeholder Group 1 Query 
Participants speculated about what type of content they may encounter on the Query Results page. One participant stated: "we will see a map of where the tweets came from", a second participant stated "I have no idea", and the third participant made a statement that we were unable to comprehend. Despite the inclusion of an introductory paragraph that described the content to be presented as information visualizations in the mock cybercartographic atlas and adjacent information timeline, participants appeared to have little foresight of the information visualizations to be presented on the Query Results page. All participants successfully located and selected the Search button, and navigated the Query Results page.

All participants interacted with the mock cybercartographic atlas and adjacent information timeline to varying degrees. We observed participants' interactions with various map assets, for example, the pop-up bus stop dialogue, which included information such as: nearest major address or intersection, bus stop amenities, a list of bus routes that service the stop, and a unique infrastructure identification number. One participant asked why the map included conventional bus stops, but omitted paratransit bus stops. We explained that developer access to a map of paratransit bus stops does not currently exist, but is something that we hope to explore further. Participants were able to access the bus stop dialogue with ease; however, when prompted to elaborate on the listed bus stop amenities, participants expressed confusion about some of the terminology used. For example, Figure 4.3 shows the pop-up bus stop dialogue box for "Carling N / Melrose (\#7365)", and lists four bus stop amenities:

1. Shelter: All participants understood what amenity "Shelter" referred to;

2. Bench: All participants understood what amenity "Bench" referred to;

3. Box: Participants did not understand what amenity "Box" referred to. *A "Box" referred to a boxed schedule display that is fixed to a flag pole; and,

4. Case: Participants did not understand what amenity a "Case" referred to. *A "Case" referred to a full or partial system map, enclosed in plastic casing. 


\section{Home > Public transit user}

Welcome to The Accessible Public Transit Infrastruture Atlas-PUBLIC TRANSIT USER view! The information presented in this atlas was harvested from the social media platform, Twitter, and is plotted spatio-temporally on a GIS style map. The map is interactive by design, and includes audio-visual media, hyperlinks, URLs, and up to 280 characters of text. You may interact with content by selecting an icon on the map or by scrolling the tweet timeline. We are actively working to geolocate as many tweets as posssible.
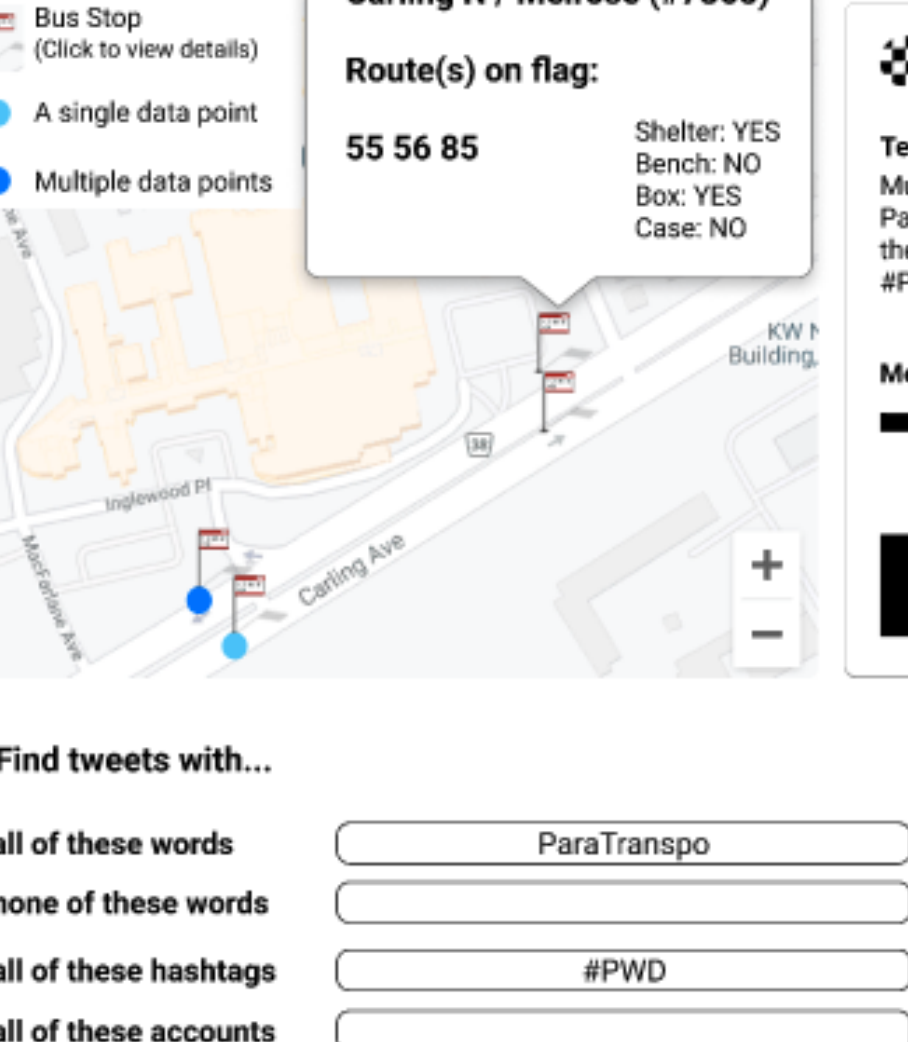

Then narrow your results with...

date range
media attachments
location

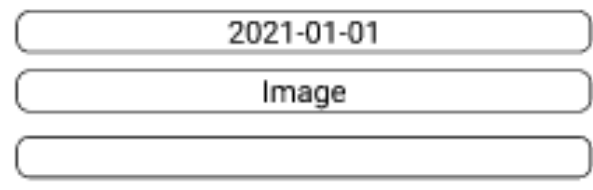

\section{@Toby}

12:11 January 12021

\section{Text}

Multiple people in my

ParaTranspo bus today... Where is the physical distancing???! \#8016 \#PWD@ottTransitRider@CBCOtt

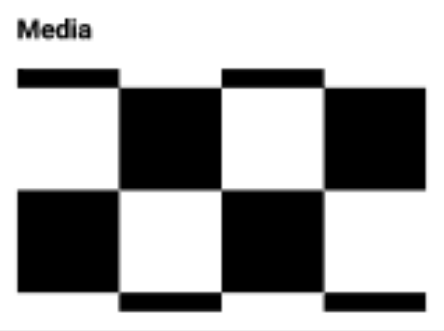

To do this in the search box.

e.g. word1, word2, word3

e.g. -word1, -word 2

e.g. \#PWD, \#ParaTranspo

e.g. @CTVnews, @CBC

To do this in the search box.

e.g. YYYY-MM-DD

e.g. slect all that apply

e.g. 3052 or Parliament

\section{Save tweet story}

Share tweet story with a community partner

Figure 4.3: Stakeholder Group 1 Results+ 
All three participants correctly identified the presence of an information timeline, adjacent to the mock cybercartographic atlas. The information timeline was prototyped as a scrollable asset, and includes a visual scroll bar in the interface design. Participants correctly identified the unique components of the information timeline: Twitter handle (e.g., @Toby), profile image, body of text, attached media (e.g., image), etc., without an explicit prompt. However, all three participants failed to recognize the scrolling feature, and therefore did not access five additional hidden Tweets - until prompted. Participants struggled to link the information displayed in the information timeline with visual icons displayed in the mock cybercartographic atlas. The final assets we evaluated in Use Case 2 include the "Save Tweet Story" and "Share Tweet Story With a Community Partner" button.

All participants correctly speculated that the 'Save Tweet Story' button would save the database query and query results, and that the 'Share Tweet Story With a Community Partner' button would send the Tweet story to a community partner. Participants described these functions as useful because: "they [community partners] need the data to ask the commission to make changes". Prior to concluding the usability-testing session, participants were provided with an opportunity to pose questions for the research team.

At the conclusion of the usability-testing session, participants were prompted with the following questions: "What do you like? What do you not like? How can the design be improved?". Participant responses included:

1. "It's actually pretty good, but maybe allow filtering using multiple key words because people spell words differently";

2. "Add curb cut information to bus stop dialogue asset";

3. "This app will bring the information directly to Transit Commission, that's where I think this thing is good, we don't want to wait for an election to change a curb";

4. "The only way we get action on things is if CBC does a story about it"; 
5. "If this thing could track the number of issues over time that would be helpful"; and,

6. "It will be easier to teach them [the Transit Commission] what its like, I think they can get a better idea of how it is, I love the idea".

\subsubsection{General Findings for Stakeholder Group 2}

We conducted remote usability-testing sessions with two participants who self-identified as members of the public transit advocate/lobby community. Participants viewed and explored a Landing page, and performed a database query task and query results exploration task. Two use cases were evaluated during the remote usability-testing session:

1. Use Case 1: View and explore the functionality of the Landing page, and select an appropriate user profile and navigate to the Database Query page; and,

2. Use Case 2: Perform a database query by entering five unique query criteria into the Database Query page, and explore the query results, visualized in a mock cybercartographic atlas and adjacent information timeline, on the Query Results page.

Following evaluation of the use cases, participants were encouraged to speak candidly about their experience participating in the remote usability-testing session, and were provided an opportunity to pose questions. At the conclusion of the remote usability-testing session, participants were asked to complete a 10-question posttest questionnaire (i.e., System Usability Scale), via email. Following completion of the post-test questionnaire, participants provided contactless delivery information, or specified a mailing address for receipt of the \$20 Tim Horton's gift card, as compensation for participating in the study. Participants reported a mean age of 41 years, and both participants identified as male.

In Use Case 1, participants viewed and explored the Landing page, shown in Figure 4.1. Both participants correctly identified the presence of a GIS style map, with a public transit infrastructure layer indicating the precise geographic location of public transit infrastructure in Ottawa. One participant described the map icon colour 
scheme as "different zones for bus stops", while the second participant described the header text as "wordy and difficult to understand". When prompted to interact with the Landing page, including the map, both participants attempted to select the ' + ' '-' icon (zoom function), embedded in the map. Participants expressed surprise when the Zoom icon did not respond as expected. After verbally describing the content of the GIS style map and exploring the map's limited functionality, both participants selected an appropriate user profile and navigated to the Database Query page.

In Use Case 2, participants performed a database query task by entering five predetermined query criteria into the Database Query page. Both participants input the five query criteria in the appropriate bars without error. Both participants expressed a degree of surprise when they attempted to use the pop-up keyboard to input the initial query criteria. We reminded participants that the purpose of the session was to evaluate the user interface and not a participant's typing skill, hence, we autocompleted the query term after the participant had selected the appropriate input bar and entered the first letter of the query criteria using the on-screen keyboard. Once a participant had successfully entered all five query criteria in the appropriate bars, they selected the Search button and navigated to the Query Results page. Prior to selecting the Search button, we paused the participant and asked them describe the content they expected to see on the Query Results page. The completed Public Transit Advocate Database Query page is shown in Figure 4.4. 


\section{Home > Accessible Public Transit Advocate}

Welcome to the Accessible Public Transit Infrastructure Atlas-ACCESSIBLE PUBLIC TRANSIT ADVOCATE view! The information presented in this atlas was harvested from the social media platform, Twitter, and is plotted spatio-temporally on a GIS style map. The map is interactive by design, and includes audio-visual media, URLs, and up to 280 characters of text. You may interact with content by selecting an icon on the map or by scrolling the tweet timeline. We are actively working to geolocate as many tweets as possible.

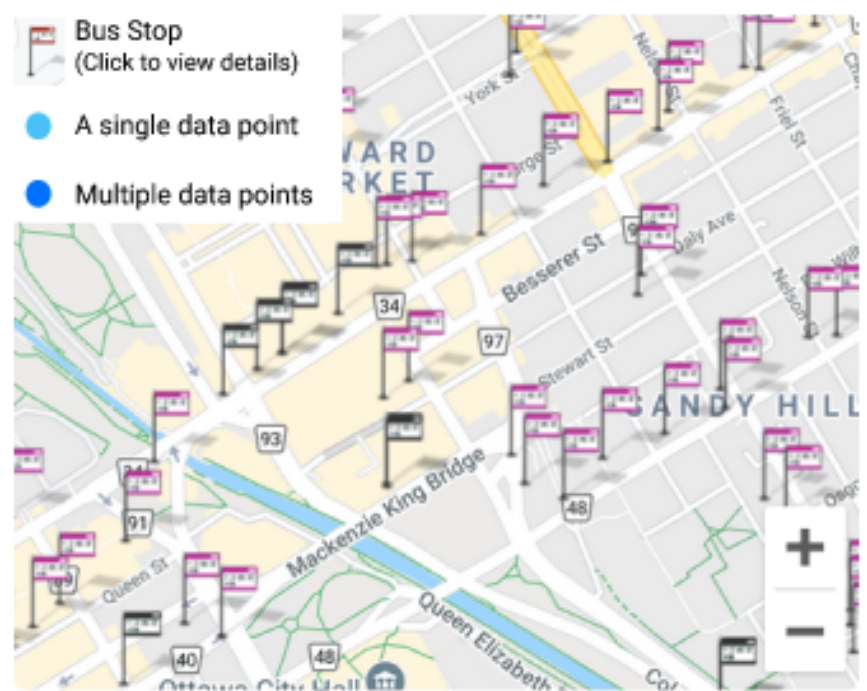

Find tweets with...

all of these words

none of these words

all of these hashtags

all of these accounts

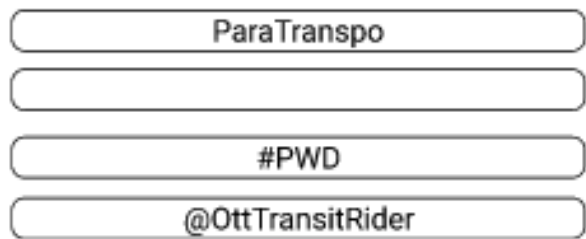

@OttTransitRider

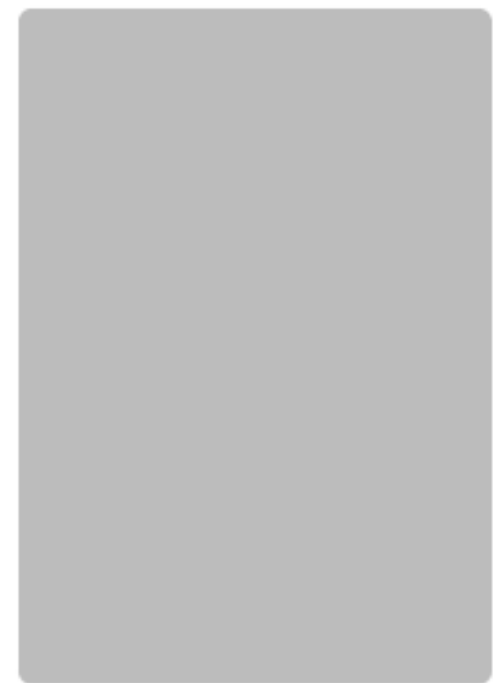

To do this in the search box.

e.g. word1, word2, word3

e.g. -word1, -word 2

e.g. \#PWD, \#ParaTranspo

e.g. @CTVnews, @CBC

To do this in the search box.

e.g. YYYY-MM-DD

e.g. slect all that apply

e.g. 3052 or Parliament

\section{Search}

Figure 4.4: Stakeholder Group 2 Query 
Both participants speculated about what type of content they expected to interact with in the Query Results page. One participant expected to see: "a band of narrow responses that meet these criteria, but its going to be very broad geographically, so you might have Tweets from Seattle because they have Paratransit over there, too", while the second participant wasn't sure what to expect other than "a GIS style map and some Tweet data". Despite the inclusion of an introductory paragraph that described the content to be presented as information visualizations in the mock cybercartographic atlas and adjacent information timeline, participants appeared to have little foresight of the information visualizations to be presented on the Query Results page. All participants successfully located and selected the Search button, and navigated the Query Results page.

Both participants interacted with the mock cybercartographic atlas and adjacent information timeline, shown in Figure 4.5. We observed participants' interactions with various map assets, for example, the pop-up bus stop dialogue, which included information such as: nearest major address or intersection, bus stop amenities, a list of bus routes that service the stop, and a unique infrastructure identification number. Similar to members of the PWD community, members of the public transit advocate lobby reported a lack of understanding when exploring the pop-up bus stop dialogue. Specifically, neither participant understood the implied meaning of "box" and "case" bus stop amenities. When participants attempted to explore the information timeline, one participant recognized the visible scroll bar, and scrolled the asset without an explicit prompt, while the second participant did not initially recognize the scroll bar, and required a prompt to scroll the asset and interact with the five hidden posts. 


\section{Home > Accessible Public Transit Advocate}

Welcome to the Accessible Public Transit Infrastructure Atlas--ACCESSIBLE PUBLIC TRANSIT ADVOCATE view! The information presented in this atlas was harvested from the social media platform, Twitter, and is plotted spatio-temporally on a GIS style map. The map is interactive by design, and includes audio-visual media, URLs, and up to 280 characters of text. You may interact with content by selecting an icon on the map or by scrolling the tweet timeline. We are actively working to geolocate as many tweets as possible.

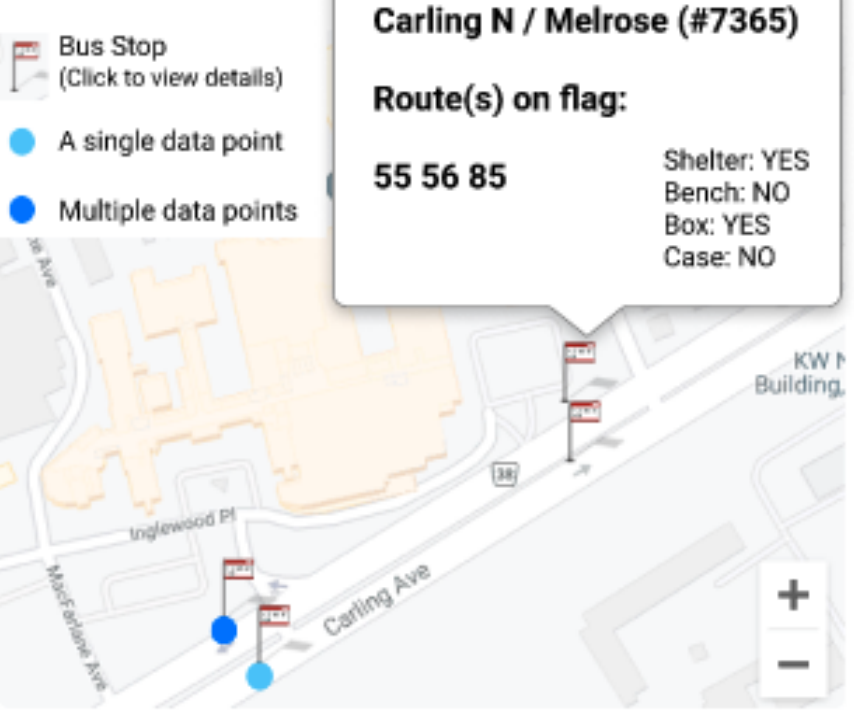

Find tweets with...

all of these words

none of these words

all of these hashtags

all of these accounts

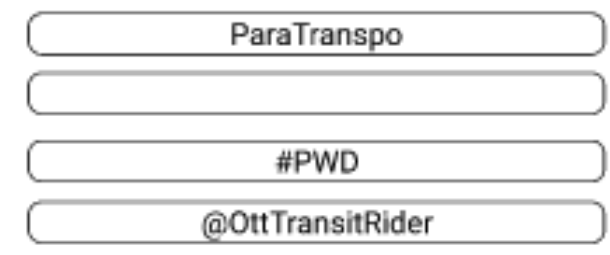

Then narrow your results with...

date range
media attachments
location

Save tweet story

\section{Add related document}

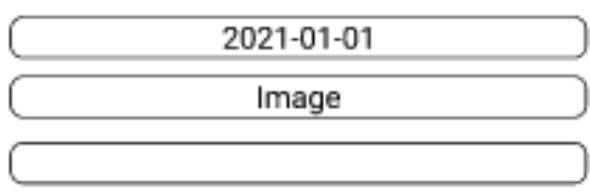

e.g. YYYY-MM-DD

e.g. slect all that apply

e.g. 3052 or Parliament

\section{(a) Toby}

$12: 11$ January 12021

Text

Multiple people in my

ParaTranspo bus today... Where is the physical distancing?!?! \#8016 \#PWD@ottTransitRider@CBCOtt

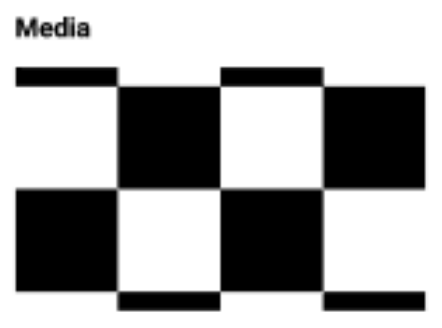

To do this in the search box.

e.g. word1, word2, word 3

e.g. -word1, -word 2

e.g. \#PWD, \#ParaTranspo

e.g. @CTVnews, @CBC

To do this in the search box.

Share tweet story

Publish for Transit Commission

Figure 4.5: Stakeholder Group 2 Query Results+ 
Both participants correctly identified the unique components of the information timeline: Twitter handle (e.g., @Toby), profile image, body of text, attached media (e.g., image), etc., without an explicit prompt. Similar to members of the PWD community, members of the public transit advocate community struggled to link the information visualized in the information timeline with visual assets included in the mock cybercartographic atlas. The final interface items evaluated in Use Case 2 include the 'Save Tweet Story', 'Share Tweet Story', and 'Publish for Transit Commission' buttons.

Both participants correctly identified the intended function of the 'Save Tweet Story' button as a feature that saves the database query and query results, by archiving an instance the query and results in the Nunaliit Cybercartographic Atlas Framework. Neither participant was able to differentiate the intended function of the 'Save Tweet Story' button from the 'Share Tweet Story' button. Both participants correctly identified the intended function of the 'Publish for Transit Commission' button, as a button that triggers the default email client to populate a predefined email, with a hyperlink to access the Tweet story, for members of the Transit Commission.

At the conclusion of the usability-testing session, participants were prompted with the following questions: "What do you like? What do you not like? How can the design be improved?". Participant responses included:

1. "It's a neat idea to start connecting some of those dots";

2. "This is a good idea to start prioritizing the placement of assets in communities that need them the most";

3. "As an advocacy group, there's the issue of, there is just so much information that stuff just gets lost through the cracks sometimes, and sorting information takes a lot of time, so this can actually be a helpful aggregator of information, and be used to drive better evidence-based policymaking";

4. "As an advocacy group, it's not enough to just complain about stuff - you need to come up with solutions too"; 
5. "Having an ability to specify solutions to a problem defined by transit riders makes us a stronger advocacy association";

6. "As you know, in academia, there is this way of writing and its not always very accessible, so I suggest that you write for a sixth-grade audience when you start to build the app. I just think that you shouldn't put up linguistic barriers"; and,

7. "You should make the language more welcoming, more clear, and more concise, because you want to try and get the broadest input you can from people using the app".

\subsubsection{General Findings for Stakeholder Group 3}

Unfortunately, the member of the Transit Commission that we spoke with during the semi-structured interview was unable to participate in the remote usability-testing session, and formally withdrew from that phase of the study. The participant did not retroactively withdraw from the semi-structured interview, and the information gathered during the semi-structured interview remains part of this thesis.

Although we did not evaluate the Public Transit Decision-Maker user profile with the participant, we include a brief discussion about the user interface design considerations, as it remains a critical component our proposed public transit decision-making IS. Figure 4.6 shows the Public Transit Decision-Maker Query page. Notably, the database query section is absent, and the user is presented with pre-determined tweet stories from recognized community partners. To access a tweet story, a public transit decision-maker must select a hyperlinked Twitter handle and view a published tweet story. Once selected, a tweet story is displayed for the public transit decision-maker as is shown in Figure 4.7 . 


\begin{abstract}
Home > Public Transit Decision Maker
Welcome to the Accessible Public Transit Infrastructure Atlas-DECISION MAKER view! The information presented in this atlas was harvested from the social media platform, Twitter, and is plotted spatio-temporally on a GIS style map. The map is interactive by design, and includes audio-visual media, URLs, and up to 280 characters of text. Please select one or more of the tabs below to view content associated with that tweet story. For exemple, if you were to select the "@OttTransitRider" tab, the map will populate with a tweet story, as curated by the community organization.
\end{abstract}

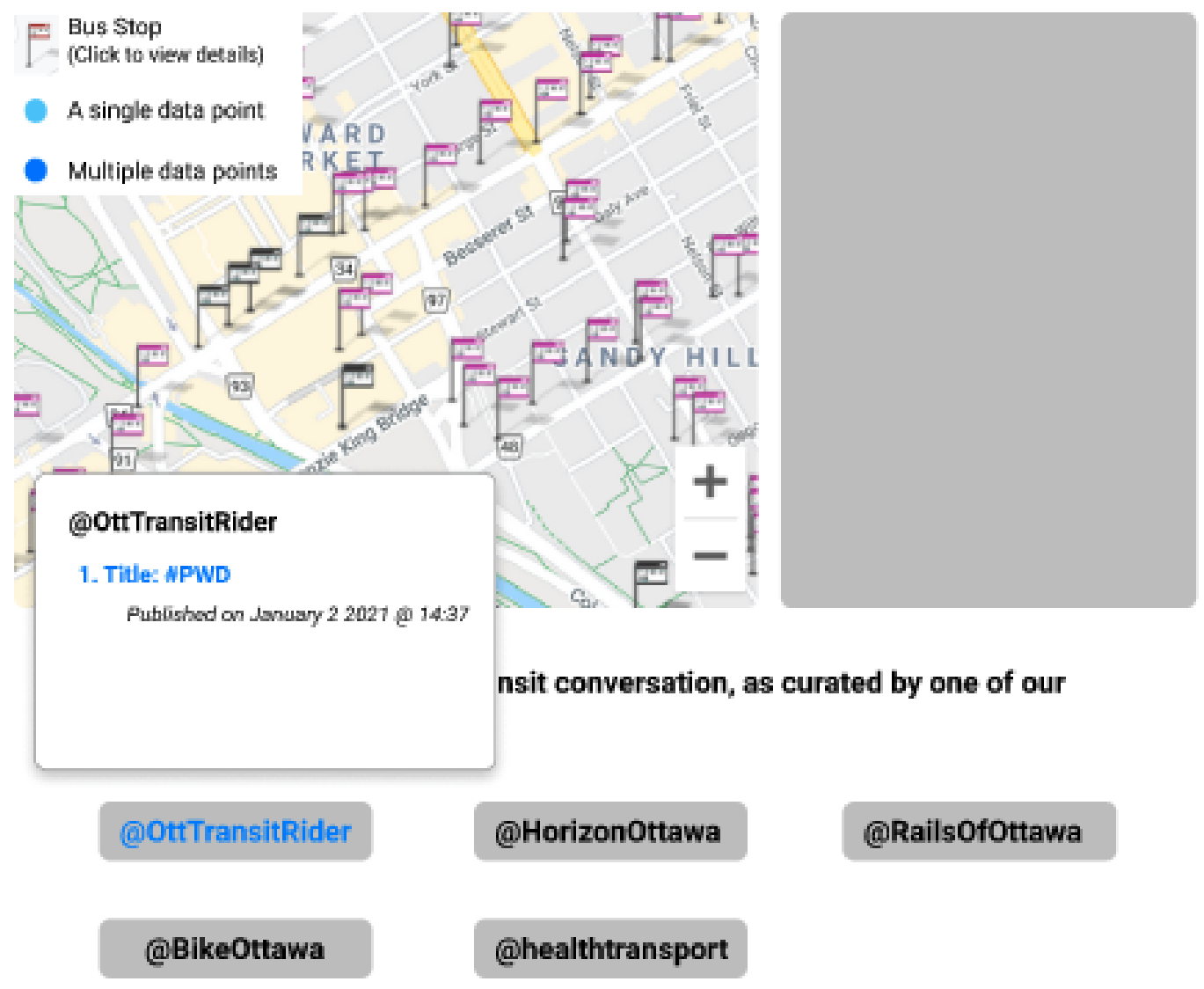

Figure 4.6: Stakeholder Group 3 Query 


\section{Home > Public Transit Decision Maker}

Welcome to the Accessible Public Transit Infrastructure Atlas-DECISION-MAKER view! The information presented in this atlas was harvested from the social media platform, Twitter, and is plotted spatio-temporally on a GIS style map. The map is interactive by design, and includes audio-visual media, URLs, and up to 280 characters of text. Please select one or more of the tabs below to view content associated with that tweet story. For exemple, if you were to select the "@OttTransitRider" tab, the map will populate with a tweet story, as curated by the community organization

Be Bus Stop
(Click to view details)

A single data point

Multiple data points

Carling N / Melrose (\#7365)

Multiple data points

Route(s) on flag:

555685

Shelter: YES Bench: NO

BoX: YES

Case: NO

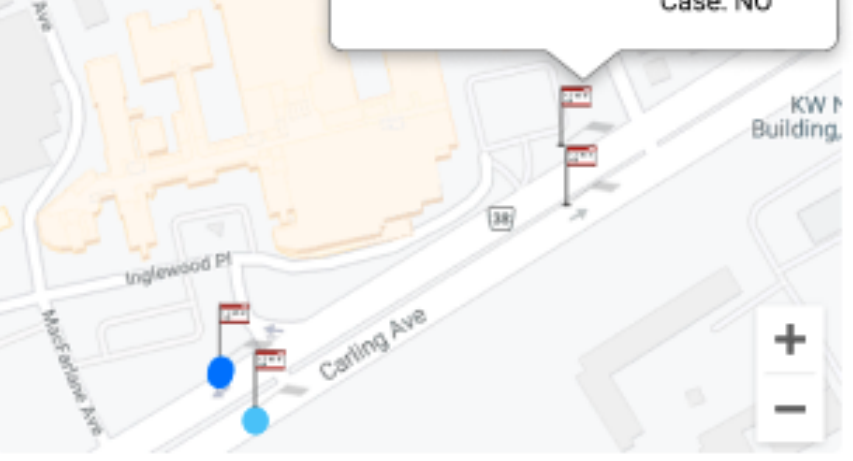

\section{Add related document}

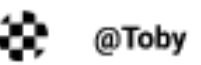

12:11 January 12021

Text

Multiple people in my

ParaTranspo bus today... Where is the physical distancing?!?! \#8016 \#PWD@ottTransitRider @CBCOtt

Media

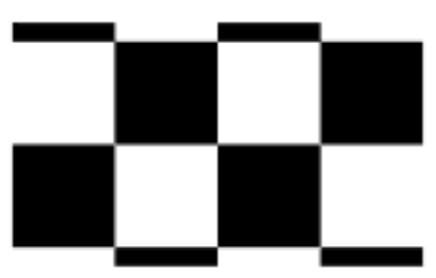

Story title: "\#PWD" submitted by: @OttTransitRider

Key information about the story here (aka talking points):

- idea 1

- idea 2

- idea 3

Proposed interim solution:

Proposed long-term solution:

Figure 4.7: Stakeholder Group 3 Results+ 
Notably, the Decision-Maker Results page includes a mock cybercartographic atlas and adjacent information timeline, and an additional text section (in-place of query input filed) with explicit expert information that compliments the of information visualizations. The additional text section is populated by the public transit advocate/lobby group that curated the tweet story. It is through this deliberate choice architecture that we hope to serve Daniel Kahneman's notion that:

"Democracy is inevitably messy, in part because the availability and the affect heuristics that guide citizens' beliefs and attitudes are inevitably biased, even if they generally point in the right direction. Psychology should inform the design of risk policies that combine the experts' knowledge with the public's emotions and intuitions" [57]. We discuss the idea of employing cartographic representations, paired with a familiar information timeline design and expert opinion, as a mechanism to improve public transit decision-making throughout the IS in chapter 5 .

\subsubsection{Post-Test Questionnaire (i.e., System Usability Scale)}

The System Usability Scale (SUS), created by John Brooke in 1986, offers a quick and effective method to evaluate perceived usability of web-based products and designs. It can be deployed across a broad range of digital products and services to determine if there is an overall problem with a design solution. The SUS is not considered a diagnostic tool and is used to provide an overall usability assessment, as defined by ISO 9241-11 [54]. The standard for the definition and measurement of usability, ISO 9241-11, suggests that "there is no specific property of an artefact that you can call usability; rather, something which is usable is something which is appropriate to its context, where the context includes the task that is being done, the background and experience of the user who is doing it, and the environment in which it is being done" [19].

Provided that usability in any given instance is defined by the context of the use of a system, it follows that, in general, the way in which usability is measured must also be defined using that same context. ISO 9241-11 breaks the measurement of usability into three dimensions that must be defined relative to the context of use: 
1. Effectiveness: Can users successfully achieve their objectives?;

2. Efficiency: How much effort and resource is expended in achieving those objectives; and,

3. Satisfaction: Was the experience satisfactory? [19].

Thus, a system that allowed a user to complete their tasks, but at the expense of a considerable expenditure of time and effort, which was felt to be very unsatisfactory by the user, can not truly be described as usable. By the same measure, however, a system which a user enjoyed using but that didn't allow them to complete their tasks, and on which they experienced a considerable expenditure of unproductive time, could equally be argued as not very usable [19].

One of the primary benefits of using the SUS is that the feedback is reliable and repeatable when deployed to five or more users in a single set of evaluations $[18,19]$. Moreover, the SUS can be used to compare different design solutions using A/B testing, provided that the order and wording of the questions does not change. We assigned a value for the SUS score calculation. The point breakdown for the responses were:

Disagree: 1 point

Somewhat Disagree: 2 points

Neutral: 3 points

Somewhat Agree: 4 points

Agree: 5 points

Scores below 68 typically indicate issues with the design that require significant additional research to be adequately resolved, while scores higher than 68 indicate the need for minor improvements to the design $[18,19]$. We reported a mean respondent score of 90.4, with five participants. The full SUS scoring breakdown is included in Table 4.1. Our objectives in administering the SUS include: 
1. To provide a measure of subjective perceptions of the usability of our system; and,

2. To allow us to do so in the very short time available to us during the remote usability-testing session.

\begin{tabular}{|l||l|}
\hline \multicolumn{2}{|c|}{ System Usability Scale Scores } \\
\hline Participant & SUS Score \\
\hline RL & 100 \\
KH & 80 \\
JR & 100 \\
TD & 85 \\
SM & 87 \\
\hline
\end{tabular}

Table 4.1: SUS Results

When we deployed the SUS to evaluate perceived usability, we made an effort to consider context and define usability using the metrics outlined in ISO 9241-11. When we considered 'effectiveness', we attempted to measure a user's subjective perception of the usability of the system; we were not looking for any diagnostic information. Had the SUS revealed a low usability score, we maintained audio/video recordings of the remote usability-testing sessions, that can be reviewed to diagnose problematic issues. When we considered 'efficiency', our objective was to avoid a scenario where the participant completes a 20-30 minute session, trying to perform a task that was frustrating or annoying, only to be further burdened with a long questionnaire. The SUS required the participant to check one box per question, for ten questions. The participant was not required to write down any opinions about anything related to the usability-testing session. The participant was able to express their opinion verbally during the debrief at the conclusion of the session, and the debrief was audio/video recorded and relevant notes are referenced above. Finally, when we considered 'satisfaction', we noted continued use of the SUS by researchers and industry alike (25 years), and view the exercise of asking a participant to complete a second SUS about the initial SUS they had just completed, as a level of recursion to far. 


\subsubsection{Challenges}

First, we must acknowledge and thank participants who took part in the remote usability-testing session for their patience and determination, as we navigated through a complete system crash, internet browser and plugin configuration issues, screen sharing errors, a technological device that was unable to run the prototype, and a low bandwidth internet connection. We describe some of the challenges experienced and how they were resolved:

1. System crash: Our MacBook Pro experienced a complete system crash and reboot during a remote usability-testing session. This was a significant event because the Zoom audio/video recording was lost, and we lost all communication with the participant for several minutes. Once we re-established the connection, the participant generously agreed to continue participating in the session, well beyond the agreed upon 45-minutes.

2. Internet browser and plugin configurations: We asked participants to access the prototype using their native browser. We proactively tested the prototype in multiple internet browsers prior to the remote-usability testing sessions, but we failed to account for custom browser plugins that could cause the prototype to malfunction. In one instance, we waited for approximately 20 minutes as a participant attempted to access the prototype using various browsers and plugin configurations. The participant found an acceptable configuration, and we proceeded with the session.

3. Screen sharing errors: Participants were asked to initiate a screen share so that we could observe them interacting with prototype on their native device. In one instance, a participant successfully initiated a screen share, however, the participant inadvertently shared a secondary screen (the participant was using a three display setup).

4. A device that was unable to run the prototype: We made a deliberate effort to build a non-resource intensive prototype that could be accessed on a computing device with limited CPU and RAM. In one instance, the participant was unable 
to access the prototype on their native device. We rescheduled the session, and the participant borrowed a device and completed the session with ease.

5. Low bandwidth internet connection: We experienced difficulty with audio/video streaming due to low bandwidth internet connections. At times, we asked participants to repeat their thought process as they completed tasks during the session. Again, we extend gratitude and thanks to those who participated in this study. 


\section{Chapter 5}

\section{Discussion}

In this thesis, we document the existing public transit decision-making IS, and conduct a preliminary evaluation of the proposed technological component of a novel IS - a database query and results visualization tool. We discuss our work in three sections:

1. A conceptual framework for the collection, organization, and visualization of public sentiment for three public transit stakeholder groups;

2. Controlling the master switch; and,

3. Cybercartography and the application of nudge architecture and behavioural insights to improve decision-making.

The six key ideas of Cybercartography, as they relate to prototype design and evaluation, are interwoven throughout this chapter. We describe the (theoretical) process of data collection via the API economy, and the organization of that data by the public transit advocate/lobby community to produce information and improve the process of public transit decision-making. During our field observations, we noted the

public transit conversation in formal and informal venues, and additionally, on the social media platform, Twitter. Our field observations highlighted several opportunities to grow citizen participation in the public transit decision-making process. The proposed IS described in this thesis is predicated on the theory and practice of Cybercartography, and use of the Nunaliit Cybercartographic Atlas Framework to improve public transit decision-making for each of the three public transit stakeholder groups we engaged with.

We address two research questions in this thesis: 
1. What are the needs of the PWD community in terms of access to public transit infrastructure, and access to the public transit decision-making process? What barriers to access exist, and what can be done to reduce the number of barriers?

2. How must the existing public transit decision-making IS change in order to incentivize community narratives over intuitive judgements, at the policymaking level?

Before we introduce discussion about the collection of public sentiment via the API economy, we must define the notions of noise and bias in human judgement, and consider the role a well-designed algorithm can play in reducing them. It is easiest to begin with an analogy of measurement because we think of judgement as a measurement that occurs using the instrument: the human mind. Suppose a judge (i.e., person making a judgment) is interested in measuring the accessibility of a bus stop using a rubric. If the judge applies the rubric to measure the same bus stop multiple times, they will most likely produce a different measurement each time, if the bus stop is complex and the rubric is well-defined. Variability (i.e., standard deviation of error) in repeated measurements on the same item, is noise. Now, suppose the judge had the bus stop measured by a scientifically precise instrument, and every time the instrument produced a measurement, there was an error. The average of these errors, is bias. Any variability in these errors, is noise.

In professional judgements of all sorts, whenever accuracy is the goal, bias and noise play the same role in the overall calculation of error [58]. In some cases, the larger contributor will be bias; in other cases, it will be noise. In every case, a reduction of noise has the same impact on overall error as does a reduction of bias by the same amount [58]. For this reason, the measurement and reduction of noise should have the same high priority as the measurement and reduction of bias. This conclusion rests on a particular approach to the measurement of error, which has a long history and is generally accepted in science and in statistics.

The method of least squares, first described in 1795 by Karl Friedrich Gauss, is a rule for scoring the contribution of individual errors to overall error [102]. Gauss's detailed arguments for his approach to the measurement of overall error are far beyond the scope of this thesis, but his measure of overall error - Mean Squared Error 
(MSE) - or the average of the squares of the individual errors of measurement, plays foundational role in the evaluation of professional judgement. The role of noise and bias in the calculation of error can be summarized in two error equations [58]:

1. Error in a Single Measurement:

$$
\text { Bias + Noise }
$$

2. Overall Error in Multiple Measurements (MSE):

$$
\text { Bias }^{2}+\text { Noise }^{2}
$$

The standard measure of overall error is the square of bias plus the square of noise, which leads to the notion that bias and noise are truly equivalent in their contribution to error [58]. The form of this equation - a sum of two squares - should be reminiscent of a high-school classic, the Pythagorean theorem. As one may recall, in a right triangle, the sum of the squares of the two shorter sides equal the square of the longest side. This suggests a simple visualization of the error equation, in which MSE, Bias ${ }^{2}$ and Noise ${ }^{2}$ are the areas of three squares on the sides of a right triangle [58]. Figure 5.1 shows how MSE equals the sum of the areas of the other two squares. In the left panel there is more noise than bias; in the right panel, more bias than noise. MSE is constant, and the error equation holds in both cases. Historically, judges have paid more attention to bias than to noise, and there is a rational for this. 

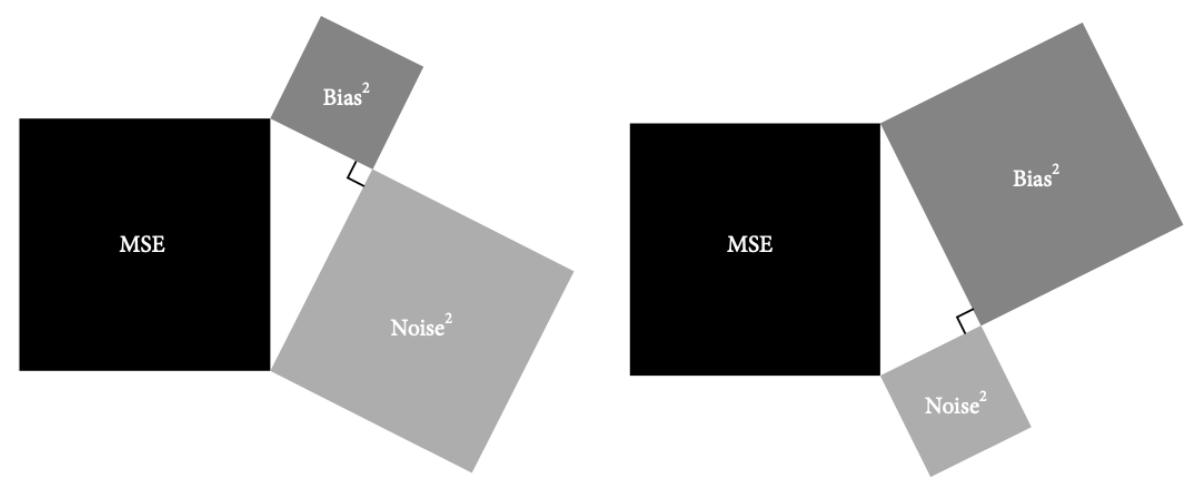

Figure 5.1: Two Decompositions of MSE

$[58]$.

There is a widespread notion that errors cancel out, which is true when measuring the same item multiple times. If a judge takes many measurements of the same item, noise will diminish because errors do cancel out. However, when a judge measures different items, errors do not cancel out. If a judge measures the accessibility one bus stop two points higher than they should have, and another bus stop two points lower than they should have, on the whole, the judge is not biassed, but they have made two very significant errors. We consider bias a force that produces a particular judgement, rather than a particular error. When we consider a particular error, we don't observe noise. Noise is a characteristic of a set of errors, as it is not possible to observe variability when considering a single measurement. This makes bias very easy to spot, and noise difficult to quantify and consider [58]. In terms of overall error, noise and bias are independent: the benefit of reducing noise is the same, regardless of the amount of bias. This notion is highly counter intuitive, but critical, and we provide a mathematical process in Appendix G.

We have judges and underwriters providing intuitive judgements at various junctures throughout the existing public transit IS, and we have a wealth of information about our built public transit infrastructure, that is potentially accessible on a handheld device. If we were to consider the wisdom-of-crowds effect, the notion that averaging several independent judgements on the same item can increase the accuracy of the resulting judgement, we can begin to reduce noise in judgments - the result being more precise judgments, evidenced by a reduction in MSE [58]. One 
straightforward way to reduce noise is to reduce judgments by imposing rules or algorithms in their place. We know that algorithms are noise free, when the same problem is passed to an algorithm multiple times, the algorithm will produce the same response, an outcome that is not true for human judges. Of course, we can be certain, at least for the next few decades, that human judgment will remain the most relied upon source for important judgments. In the following sections, we describe how a carefully curated dataset can be deployed to judges within an IS, who can, in turn, exercise judgement, without explicit awareness of the truly bad outcomes (i.e., edge cases) they avoided, thanks in-part to a well-defined algorithm that collects and organizes multiple independent measurements on the same item, for the judge.

\subsection{A Conceptual Framework for the Collection, Organization, and Vi- sualization of Public Sentiment for Three Public Transit Stakeholder Groups}

A conceptual schema is a map of concepts and their relationships that provides a structural background and explains the conceptual structure of, and development frameworks for, an information system [47]. In chapter 2, we described the conceptual structure and developmental frameworks of the Nunaliit Cybercartographic Atlas Framework; in chapter 3, we define two core database functions that were evaluated in remote usability-testing sessions (i.e., database query and results exploration), and describe the theoretical process of building a high-quality filter, using Twitter API. Previous applications of Cybercartography have included social media data in their representations of information; however, that data has always been manually entered by researchers, or community members in partnership with researchers [110].

The social media revolution has created an opportunity for researchers to access the data required for rational economic planning, data that are distributed among individual actors, and thus unavoidably exist outside the knowledge of a central authority. The Nunaliit Cybercartographic Atlas Framework provides a metadata structure that can facilitate the collection, organization, and visualization of the data required to exercise sound judgment in rational economic planning activities through its data handling capacity that can be described as a system of tolerated difference. 
During our field observations, and additionally, while conducting the semi-structured interview, we observed a pattern in which social media data is used in the course of exercising professional judgement. The public transit advocate/lobby community described a process of learning about barriers to access public transit infrastructure and barriers to access public transit decision-making, via singular (but often viral) social media interactions and observations.

In many instances, the judge became aware of a barrier to access via a viral social media post (i.e., a post with elevated engagement metrics, e.g., likes, retweets, etc.). Virality of a single measurement is not a substitute for consideration of multiple independent measurements on the same item, and any judgment made using a single measurement that happened to go viral is inferior to a judgment that considers multiple independent measurements on the same item. Moreover, if we accept the premise: in social media environments, the user's attention is the product for sale, then we must conclude that edge cases make up the vast majority of viral content, thereby making a market for disinformation (i.e., information that deviates from the mean we expect to observe when multiple measurements on a single item are judged together) [129, 82]. A well-designed cybercartographic product can mitigate this type of error in human judgment through carefully constructed information visualizations that consider multiple sets of independent measurements on multiple items an outcome that is possible when a set measurements on the same item is organized temporal-spatially. Judgments that consider multiple independent measurements on the same item serve the goal of reducing system noise in predictive judgment, a goal that is in principle as important as the reduction of statistical bias (i.e., the average error in a series of measurements) [58].

The error equation and the conclusions drawn from it depend on use of MSE as the measure of overall error. The rule is appropriate for purely predictive judgments, including forecasts and estimates, all of which aim to approach a true value with maximum accuracy (the least bias) and precision (the least noise) [58]. Good policymaking should be based on objective and accurate predictive judgments that are completely unaffected by hopes and fears, or by preferences and values. A public transit decision-maker must weigh many options when deciding how best to allocate the 
800MM annual Transportation Budget [27]; considerations such as determining and meeting the needs of residents and visitors, providing excellent customer service and fostering a reputation of safety and reliability, providing a transit service that is accessible and affordable, etc., is a matter of exercising sound judgment. A government responding to a health crisis, such as a pandemic, must weigh the pros and cons of various options, but no evaluation of options is possible without accurate predictions about the likely consequences of each option (including the option to do nothing, i.e., maintain the status quo). Predictive judgments can be improved by procedures that reduce noise, so long as they do not increase bias to a larger extent [58].

Consider this question: what percentage of people feel the urge to relieve themself while embarking on a journey that includes use of a public transit system? As you think about the question, an answer probably comes to mind. But the answer does not occur to you in the same way that you remember your birthday or your phone number. You are aware that the number in your mind is an estimate - it is not a random number. But the number in your mind is simply one estimate in a range of possible estimates that you would not rule out. If someone added or subtracted 2 percentage points from your estimate, you would most likely not find the new estimate much less plausible than your own.

Two psychologists, Edward Vul and Harold Pashler, had the idea of asking people to answer this sort of question not once but twice. In their experiments, the subjects were not informed during the first session that they would be asked to provide a second estimate at a later time. Vul and Pushner's hypothesis was that the average of the two estimates would be more accurate than either of the estimates on its own. In several independent experiments, Vul and Pulshner found that, in general, the average of two guesses from one individual (i.e., within-person average) was more accurate (i.e., lower mean squared error) than either guess on its own, and further, that the first guess was closer to the true value than the second $[57,123]$.

Similar results have been found in hundreds of estimation experiments over the past century [58]. Of course, if the questions are so difficult that only experts can come close to the answer, crowds will not necessarily be very accurate. But, for instance, when people are asked to predict the utility of a public restroom situated 
in public transit system, the average answer of a large number of people is likely to be closer to the true value than an answer that involves zero public consultation (as was the case for the LRT), or an insignificant amount of public consultation, as is the existing practice at the City of Ottawa with respect to determining the needs of the PWD community [29]. As one may recall, we defined a municipal working group as: a small cohort of individuals, typically 4-8 persons, who are consulted at regular intervals by city planners, in order to provide insight about the needs of a community. We provide a simplified analysis of the decision-making process that was used to determine restroom requirements for the new 2.1B Confederation Line, below.

In 2011, the Finance and Economic Development Committee approved the provision of two public restrooms in Stage 1 of LRT construction, as mandated in Ontario Building Code 3.13.6.2 "Washrooms Required" [59]. This decision maintained the status quo - it met the minimum requirement. "During debate and discussion on this report staff responded to Councillor questions on public washrooms clarifying that there was no plan to include additional public washrooms on the system." [59]. Later, in December 2015, the same committee made a determination that the initial decision re: public restrooms "be amended to include the planning and construction of accessible, integrated public washrooms within the fare-gated areas of the Bayview and Hurdman stations" [59]. The amendment effectively doubled the number of public restrooms in the system from two to four - and, as is indicated under the heading 'Consultation': "There was no [public] consultation undertaken for this report." [59]. However, upon further investigation, we became aware of external lobbying efforts undertaken by Ottawa's 'GottaGo' advocacy group - "We've won some very important victories in terms of toilets at Bayview and Hurdman." [92].

The database query and results visualization tool described in this thesis is designed to facilitate the collection and organization of public sentiment on various public transit issues, as identified by the PWD community. MI theory contends that individuals have different leaning preferences and prefer teaching and learning materials in different formats [41]. Cybercartographic atlases have great potential in both formal and informal education, as they provide the same information in multiple formats and modalities [110]. Many topics of interest to society are complex 
and the same set of 'facts' are open to a wide variety of representations. Even when there may be some agreement on the facts, there can often be a wide variety of interpretations. There are often no simple 'right' or 'wrong' answers to many questions. Cybercartography allows the presentation of different ontologies or narratives on the same topic, without privileging one over another. The user is able to engage with the various narratives presented and form a deeper understanding of the complexities and uncertainties associated with each perspective. Traditional public transit planning in Western societies is an authoritative top-down exercise, headed by municipal governments [124]. Cybermaps are much more nuanced - they provide a canvas to warn of the mistakes of equating money with capital and divorcing economic capital from its social partner.

\subsection{Controlling the Master Switch}

During our field observations, we noted how public sentiment often travelled through traditional media organizations before it was considered/debated by public transit decision-makers at Ottawa City Hall. The emergence of the Covid-19 Global Pandemic highlighted a significant vulnerability in the existing public transit decisionmaking IS: namely, dependence on traditional media organizations to communicate community issues, or share community narratives, cannot be assured, especially when a new breaking story emerges. This is not to suggest that community partnerships with traditional media organizations should be abolished altogether, but rather, the public transit decision-making IS should be independent of managing editors whose mandate is to retain user attention at all costs. Prior to the emergence of the Covid19 Global Pandemic, public sentiment amplified by the public transit advocate/lobby community received regular media coverage, and this coverage highlighted various public transit issues (i.e., viral social media posts that often included audio/video media) for public transit decision-makers. As of March 2020, PWD narratives received little media coverage, as the media landscape shifted to what was at the time, an emerging mega-story, sprawling in every possible direction. Our proposed database query and results visualization tool can mitigate this vulnerability by leveraging core database functions in the Nunaliit Cybercartographic Atlas Framework to establish 
a direct channel for effective communication of public sentiment, visualized through community narratives, organized by the public transit advocate/lobby, for public transit decision-makers.

During the semi-structured interview, members of the PWD community expressed a desire to share their experiences using the public transit system directly with public transit decision-makers, via social media interactions. When we engaged with a single public transit decision-maker during the semi-structured interview, they described the practice of using social media posts as a testamentary instrument in official decision-making activities as a "double-edged sword", due to the "exaggerated nature of social media content". We agree that the use of viral social media posts in course of exercising professional judgment is not conducive to accurate decision-making. Accordingly, we implemented a 'Share Tweet Story with a Community Partner' button that enables a user to submit a collection of social media posts (i.e., tweet story potentially including a viral post, or not), organised temporal-spatially, to the public transit advocate/lobby community. The public transit advocate/lobby community user profile features additional functions such as the ability to contribute non-social media content to any existing tweet story, and the ability to publish a tweet story for members of the Transit Commission.

During the semi-structured interview, members of the public transit advocate/lobby community expressed a desire to aggregate various documents (e.g., media publications, blog posts, Town Hall notes, etc.) with an underlying public sentiment dataset. Accordingly, we implemented an 'Add Related Document' button that enables a user to upload virtually any type of additional media to an underlying dataset, i.e., tweet story (this is possible due to the robust data handling functions of the Nunaliit Cybercartographic Atlas Framework, described in chapter 2). Figure 4.3 shows the Query Results page with the 'Add Related Document' button located above the information timeline, adjacent the mock cybercartographic atlas. The inclusion of this button on the Query Results page was a deliberate design choice - the rational being, the addition of related media should compliment an existing dataset produced via a database query. 
Finally, in order to ensure an uninterrupted cycle of community narrative originating with the PWD community, travelling through the public transit advocate/lobby community, and ending in the hands of public transit decision-makers, we implemented a 'Publish for Transit Commission' button in the 'Query Results' page of the 'Public Transit Advocate/Lobby' user profile. When selected, the button will trigger a mailto:Transit Commission function, and auto-generate a hyperlink that directs to a saved Query Results instance (including any additional related documents). All members of the Transit Commission will receive an email and link to access the cybercartographic atlas, complete with interactive data visualizations.

\subsection{Cybercartography and the Application of Choice Architecture and Behavioural Insights to Improve Decision-Making}

Cass R. Sunstein and Richard H. Thaler have worked many of the core ideas that underpin Nudge theory. They argue that people are quite susceptible to counterproductive behavioural and cognitive tendencies, therefore, it is appropriate for social planners and policymakers to modify decision-making situations in ways that nudge people toward better choices [114]. They distinguish 'nudging' from 'forcing' by the fact that people can still choose the de-emphasized option relatively easily. We apply the concepts of nudging and choice architecture in the design of a database query and results visualization tool, that serves the decision-making needs of three public transit stakeholders in Ottawa. We leverage the core database functions of the Nunaliit Cybercartographic Atlas Framework to collect and organize public sentiment in a manner that serves to nudge decision-makers toward better decision-making outcomes.

In historical applications, including 'government nudge units', nudging and choice architecture has been used to induce a desired outcome (i.e., option A vs. option B) [114]. In our prototype, nudging and choice architecture is used to induce a desired process. The choice architecture deployed in the Database Query page is designed to nudge the user to consider a set of judgments on a single item, and where appropriate, consider temporal-spatial relationships in a set of judgments. Sets of judgments on a single subject, visualized together, allow a user to form a more holistic understanding of the subject. Educational theory suggests that individuals learn best when when 
they are actively, rather than passively, involved [41, 110]. A user remains actively involved with the information visualizations represented on the Query Results page by manipulating the query fields in the lower tier of the page. A user is able to modify their initial query using keywords, hashtags, accounts, etc., while maintaining a visual of any changes to the data represented in the cybercartographic atlas and adjacent information timeline.

In May 2021, Daniel Kahneman, Olivier Sibony, and Cass R. Sunstein published a set of mathematical equations that can be used to quantify noise in human judgment - the set of error equations referenced earlier in this chapter. Kahneman et al. refer to the process of quantifying noise in a set of judgments as: conducting a 'noise audit'. In the remote usability-testing session, we asked participants to explore popup bus stop dialogues, which included information such as: nearest major address or intersection, bus stop amenities, a list of bus routes that service the stop, and a unique infrastructure identification number. Participants were then asked to judge of the utility of the bus stop assets listed in each unique bus stop dialogue box. Figure 5.2 shows four unique bus stop dialogue boxes that were evaluated during the remote usability-testing session. Our sample size was too small to consider conducting a noise audit; however, we note the possibility of exploring the potential to conduct a noise audit, using a larger sample, at a future time. The benefit of taking action to reduce noise in professional judgments is an increase in the precision of the resulting judgment, as well as increased awareness of bias [58]. In professional judgements of all sorts, whenever accuracy is the goal, a reduction of noise should be considered, even when the true outcome is unknown.

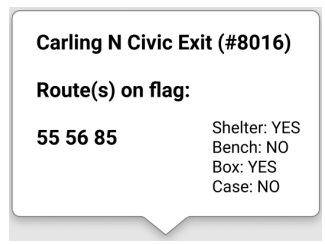

(a) Bus Stop 8016

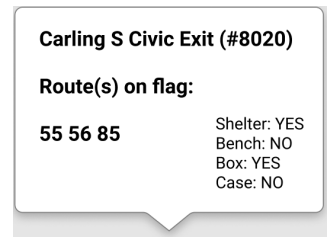

(b) Bus Stop 8020

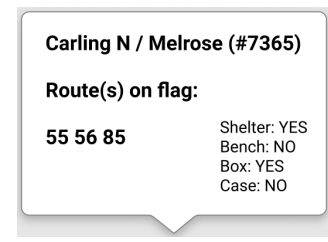

(c) Bus Stop 7365

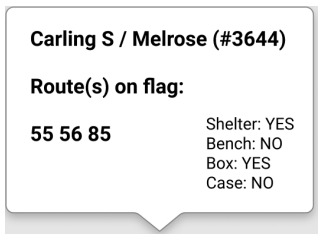

(d) Bus Stop 3644

Figure 5.2: Four Unique Bus Stop Dialogues

Traditional cartography was a supply driven industry. National mapping agencies 
supplied the definitive and authoritative maps, which the public used [110]. Technological advances have allowed for a much more demand driven approach to mapping, and Cybercartography takes this one step further by empowering individuals and communities to define the structure and data relations of a map, including the choice of what information is represented [110]. During the remote usability-testing sessions, we asked participants what information they would like represented on a cybercartographic atlas that includes a base layer indicating the location of all public transit infrastructure in the City of Ottawa. Participant responses are summarized in chapter 4; two notable items include: information about curb cuts, and the location of paratransit-only bus stops. We intend to work with the PWD community to add the relevant information to the existing public transit infrastructure map, currently available via the City of Ottawa API (i.e., Open Ottawa).

The choice architecture deployed in the Public Transit Decision-Maker user profile is designed to nudge the user to consider a set of judgments on a single item, and where appropriate, consider temporal-spatial relationships in that set of judgments. During the semi-structured interview, the public transit decision-maker noted an inability to sort through large volumes of social media content in order to learn about issues important to the PWD community. They described a case of information overload. They also described the process of using singular social media posts as a testamentary instrument in official decision-making activities as a "doubled edged sword", due to "the exaggerated nature of social media content". Accordingly, we offloaded the database query task to the Public Transit Advocate/Lobby community, and designed a dashboard to host tweet stories curated by the public transit advocate/lobby community, for public transit decision-makers. As one may recall, the Public Transit Advocate/Lobby user profile also features a button to add related documents, information that is related to the underlying dataset, but derived from a non-social media source.

Consider the research question: How must the existing public transit decisionmaking IS change, in order to incentivize community narratives over intuitive judgements, at the policymaking level? Individuals use all of their senses observing what is around them. If a cybercartographic product is designed to convey a community 
narrative, it should explore all possibilities of using all five senses in its representations in order to be as effective as possible, for the widest audience possible [110]. A multimodal approach, as described in chapter 2, maximizes available communication channels. Moreover, the user must have assurance that the information representations are not subject to any legal action, or in violation of any applicable intellectual property and privacy laws, or other civil liberty violations, as described in chapter 2 . Taylor and his research team have worked many of the early problems that presented in the iterative and holistic development between the theory and practice of Cybercartography; the value of cybercartographic products continues to increase with each iteration.

How can we quantify the value of a cybercartographic atlas, deployed as the core technological component of a novel public transit decision-making IS? Concepts of value are rooted in philosophy, and more recently and narrowly, in economic and financial theory [22]. Values and value are related, but distinct. Values represent principles or standards of behaviour, they are judgments of what is important: in the context of a public transit system, decision-makers should strive for the provision of excellent customer service and a reputation of safety and reliability, and a transit service that is accessible and affordable. Value is the regard that something is held to deserve, its importance, its worth, its usefulness. Value is not necessarily constant but, rather, specific to time and situation [22].

Consider the value of bench at a bus stop. The bench situated outside a retirement residence provides great value to its community, relative to the bench situated in front of a single detached home in a wealthy neighbourhood. Yet, when we review the City of Ottawa's Comprehensive Asset Management Policy Framework, State of the Asset Report (2017), and Strategic Asset Management Plan (2017), we find that the value of a bench is equal to its capital cost, there is no mention of context. We can observe the bench's value as it depreciates over time, and at some point in its life cycle, its value is equated with a cost of replacement. A cybercartographic atlas, deployed in a municipal decision-making environment, provides a canvas, from which the social value of our build environment can be communicated through interactive information representations that take context into consideration. Adam Smith's magisterial works 
(i.e., The Theory of Moral Sentiments and The Wealth of Nations) warned of the mistakes of equating money with capital and divorcing economic capital from its social partner; we view Cybercartography as a practice that serves the goal of exploring the value of things from the perspective of the community. 


\section{Chapter 6}

\section{Conclusion}

\subsection{General Findings}

We passively observed activities such as Town Halls, City Council and Transit Commission meetings, visited major transit hubs during peak service hours, and observed the public transit conversation happening on the social media platform, Twitter. Our observations from these activities allowed us to begin documenting the existing public transit decision-making IS. We learned about issues of importance to public transit users in Ottawa, and we documented the way in which public transit issues are discussed in various formal and informal environments.

We used the information gathered during field observations to define three public transit stakeholder groups, and proceeded to conducted semi-structured interviews with each group. During the semi-structured interview, we increased our understanding of participant interactions with public transit infrastructure, and participant use of the social media platform, Twitter, to participate in the ongoing public transit conversation happening on the platform. We were able to map the bi-directional flow of information between the public transit user, the public transit advocate/lobby, and the public transit decision-maker. We pivoted our initial methodology to reflect available resources and remote usability-testing constraints during the Covid-19 Global Pandemic. We used 'figma', a vector graphics editor and prototyping tool, to design a low-fidelity prototype in place of a proper cybercartographic atlas with limited functionality. We designed a series of wireframes with 100+ unique components, and linked sets of wireframes sequentially to produce a low-fidelity prototype, with a mock cybercartographic atlas as the central artefact. We defined two use cases, and evaluated our prototype using a database query and a results visualization task.

The remote usability-testing session provided an opportunity to gain valuable insight about the prototype design. The sessions were audio/video recorded, and we 
reviewed the recordings to diagnose system-wide user interface issues. We identified two issues that require immediate design solutions:

1. All participants were prompted to read the header text included on the Landing page, Database Query page, and Query Results page. Participants were paused at different junctures, and asked to speculate about what type of information they expected to encounter on a subsequent page. Despite reading the header texts, participants were unable to provide meaningful insight about subsequent pages. Following a detailed analysis of the usability-testing sessions, we employed a content grade tool to assess the reading level of the texts and found the texts to be at grade 11-12 reading level. Our initial design goal was to include text that was accessible to users at an eighth grade reading level. We failed to adequately evaluate the reading level of the text included in the prototype, prior to deploying the prototype in the initial remote usability-testing session.

2. We observed participants as they entered pre-determined query criteria into the Database Query page. Although participants completed the task with few input errors, we recognized a significant design issue on the Database Query page. The interface design we evaluated was modelled after the advanced Google search form fill design (i.e., standard academic database query design), and included a series of data entry fields (i.e., search bars), separated by two headers: 'Find tweets with...' and 'Then narrow your results with...' Participants appeared to have some understanding they were performing a database query that would return public tweet data, retrieved via Twitter API. However, multiple participants expressed a misguided belief that they could use the system to query all Twitter data. For example, one participant stated that they could use to system to find Tweets about their favourite musical band. This is not the case, as the database query function is restricted to pulling data that directly from the Nunaliit Cybercartographic Atlas Framework. A potential design solution should restrict a user's initial query capability by implementing drop-down menus (similar to the media attachment drop down bar) in place of text input bars. 
In addition to reviewing the audio/video recordings from the remote usabilitytesting sessions to diagnose specific usability issues, we asked participants to complete a 10-question post-test questionnaire (SUS). We deployed the SUS to assess a participant's perception of the usability of the system, in a $<5$ minute window, at the conclusion of the remote usability-testing session. We tabulated a mean SUS score of 90.4 across five participants, representing two stakeholder groups. A SUS of 90.4 suggests that participants perceived the system to be usable in the contexts we evaluated. A SUS usability score is intended to reflect perceived usability through the lens of effectiveness, efficiency, and satisfaction.

\subsection{Lessons Learned}

We chose to conduct semi-structured interviews and remote usability-testing sessions with multiple participants who reported living below the poverty line, and other participants who served as elected or appointed municipal officials. This decision introduced significant challenges in terms of accessing the participant group for the purpose of the semi-structured interview and remote usability-testing session, as highlighted in chapter 4 . For the purposes of a graduate thesis, selecting a more accessible participant group is ideal.

\subsection{Limitations and Future Considerations}

Our work has created some foundational knowledge, from which many new problems can be worked. We chose to work exclusively with a Twitter dataset, and provided strong reasoning for that decision. We recognize the limitations of relying on data retrieved via the API economy. There is always risk when a data source is derived from a for profit company. We also recognize that findings derived from Twitter data are not generalizable to the general population, as many people chose not to use social media. Merging multiple data sources is a consideration for future work. 


\section{References}

[1] Ahlberg, C., And Shneiderman, B. Visual information seeking: Tight coupling of dynamic query filters with starfield displays. In The craft of information visualization. Elsevier, 2003, pp. 7-13.

[2] Alam, F., Ofli, F., And Imran, M. Crisismmd: Multimodal twitter datasets from natural disasters. In Proceedings of the International AAAI Conference on Web and Social Media (2018), vol. 12.

[3] Anderson, J. C., Lehnardt, J., And Slater, N. CouchDB: The Definitive Guide: Time to Relax. "O'Reilly Media, Inc.", 2010.

[4] Anonby, E., Murasugi, K., And Dominguez, M. Mapping language and land with the nunaliit atlas framework: Past, present and future. Proceedings of FEL XXII (2018): Endangered Languages and the Land: Mapping Landscapes of Multilingualism (2018).

[5] BACON, B. Message from the president: Maintaining required services and successfully completing the term.

[6] Baddeley, A. The concept of working memory: A view of its current state and probable future development. Cognition 10, 1 (1981), $17-23$.

[7] Baddeley, A., And Logie, R. Auditory imagery and working memory.

[8] Barrass, S., And Kramer, G. Using sonification. Multimedia Systems 7, 1 (1999), 23-31.

[9] Baulch, S., Macdonald, R., Pulsifer, P. L., And Taylor, D. F. Cybercartography for education: The case of the cybercartographic atlas of antarctica. In Modern Cartography Series, vol. 4. Elsevier, 2005, pp. 491-515.

[10] Bederson, B. B., And Hollan, J. D. Pad++ a zooming graphical interface for exploring alternate interface physics. In Proceedings of the 7th Annual ACM Symposium on User Interface Software and Technology (1994), pp. 17-26.

[11] Bertin, J. Semiology of graphics; diagrams networks maps. Tech. rep., 1983.

[12] Billinghurst, M. Put that where? voice and gesture at the graphics interface. ACM Siggraph Computer Graphics 32, 4 (1998), 60-63.

[13] Blattner, M. M. Sonic enhancement of two-dimensional graphics displays. Auditory Display-Sonification, Audification and Auditory Interfaces (1994), 447-470. 
[14] Bosman, S., Groenendahl, B., Findlater, J.-W., Visser, T., DE Graaf, M., And Markopoulos, P. Gentleguide: An exploration of haptic output for indoors pedestrian guidance. In International Conference on Mobile Human-Computer Interaction (2003), Springer, pp. 358-362.

[15] Bostock, M. Data-driven documents.

[16] Brauen, G., Pyne, S., Hayes, A., Fiset, J.-P., And Taylor, D. F. Encouraging transdisciplinary participation using an open source cybercartographic toolkit: The atlas of the lake huron treaty relationship process. Geomatica 65, 1 (2011), 27-45.

[17] Brewster, S. A. Providing a structured method for integrating non-speech audio into human-computer interfaces.

[18] Brooke, J. Sus: a “quick and dirty' usability evaluation. Usability Evaluation in Industry 189 (1996).

[19] Brooke, J. Sus: A retrospective. Journal of Usability Studies 8, 2 (2013), 29-40.

[20] Budhathoki, N. R., Nedovic-Budic, Z., et Al. Reconceptualizing the role of the user of spatial data infrastructure. GeoJournal 72, 3-4 (2008), 149-160.

[21] Carleton University. Distinguished research professor fraser taylor FRSC. Geography and Environmental Studies (April 1 2021).

[22] Carney, M. Values: Building a Better World for All. Signal, 2021.

[23] Cartwright, W. E. Linking geographical facts with cartographic artifacts. Cybercartography: Theory and Practice (2005), 331-348.

[24] City of Ottawa. Transit commission meeting. Minutes 7 (2019).

[25] City of Ottawa. Transit commission meeting. Minutes 8 (2019).

[26] City of Ottawa. Transit commission meeting. Minutes 9 (2019).

[27] City of Ottawa. Ottawa city council youtube channel.

[28] City of Ottawa. Mandate, membership, and matters of importance. City of Ottawa (March 17 2020).

[29] City of Ottawa. Mandate, membership, and matters of importance. City of Ottawa (March 17 2020).

[30] Cohen, P. R. The role of natural language in a multimodal interface. In Proceedings of the 5th Annual ACM Symposium on User Interface Software and Technology (1992), pp. 143-149. 
[31] Cohen, P. R., And Oviatt, S. L. The role of voice input for human-machine communication. Proceedings of the National Academy of Sciences 92, 22 (1995), 9921-9927.

[32] Colby, G., And Scholl, L. Transparency and blur as selective cues for complex visual information. In Image Handling and Reproduction Systems Integration (1991), vol. 1460, International Society for Optics and Photonics, pp. 114-125.

[33] Crawford, B. 'i'm sorry. i'm sorry. i'm sorry.' red-vested oc transpo ambassadors on the front line of lrt chaos. Ottawa Citizen (2019).

[34] Cronly-Dillon, J., Persaud, K., and Gregory, R. The perception of visual images encoded in musical form: a study in cross-modality information transfer. Proceedings of the Royal Society of London. Series B: Biological Sciences 266, 1436 (1999), 2427-2433.

[35] Dewey, J. Experience and education. In The Educational Forum (1986), vol. 50, Taylor \& Francis, pp. 241-252.

[36] Duffy, A. Mayor watson makes peace in twitter war, says he will unblock people. Ottawa Citizen (November 2 2018).

[37] EdDy, B. G., AND TAYLOR, D. Exploring the concept of cybercartography using the holonic tenets of integral theory. Cybercartography: Theory and Practice (2005), 35-61.

[38] Elwood, S. Volunteered geographic information: Key questions, concepts and methods to guide emerging research and practice. GeoJournal 72, 3 (2008), $133-135$.

[39] Fisher, P. F. Hearing the reliability in classified remotely sensed images. Cartography and Geographic Information Systems 21, 1 (1994), 31-36.

[40] Gardner, H. E. Multiple Intelligences: New horizons in Theory and Practice. Basic books, 2008.

[41] Gardner, H. E. Frames of Mind: The Theory of Multiple Intelligences. Hachette Uk, 2011.

[42] Geomatics and Cartographic Research Centre. Nunaliit atlas framework.

[43] Gibbon, D., Mertins, I., And Moore, R. K. Audio-visual and multimodal speech-based systems. In Handbook of Multimodal and Spoken Dialogue Systems. Springer, 2000, pp. 102-203. 
[44] Goodchild, M. F., And Glennon, J. A. Crowdsourcing geographic information for disaster response: A research frontier. International Journal of Digital Earth 3, 3 (2010), 231-241.

[45] Griffin, A. L. Feeling it out: The use of haptic visualization for exploratory geographic analysis. Cartographic Perspectives, 39 (2001), 12-29.

[46] HAKLAY, M. How good is volunteered geographical information? a comparative study of openstreetmap and ordnance survey datasets. Environment and Planning B: Planning and Design 37, 4 (2010), 682-703.

[47] Halpin, T., AND Morgan, T. Information Modeling and Relational Databases. Morgan Kaufmann, 2010.

[48] Harden, J. Transit town hall. Community Town Halls Transit Town Hall (2019).

[49] Hardy, D. Discovering behavioral patterns in collective authorship of placebased information. Internet Research 9 (2008), 15-18.

[50] Hauptmann, A. G. Speech and gestures for graphic image manipulation. In Proceedings of the SIGCHI Conference on Human Factors in Computing Systems (1989), pp. 241-245.

[51] HAYEK, F. A. The use of knowledge in society. Library of Economics and Liberty (1945).

[52] Hedley, N. Empirical evidence for advanced geographic visualization interface use. In International Cartographic Congress, Durban, South Africa (2003), Citeseer.

[53] Holland, S., Morse, D. R., And Gedenryd, H. Audiogps: Spatial audio navigation with a minimal attention interface. Personal and Ubiquitous Computing 6, 4 (2002), 253-259.

[54] International Organization FOR Standardization. Ergonomics of human-system interaction-part 11: Usability: Definitions and concepts. Online Browsing Platform (2021).

[55] Jacobson, R. D., Kitchin, R., And Golledge, R. G. Multi-modal virtual reality for presenting geographic information. Virtual Reality in Geography (2002), 382-400.

[56] Judge, E. F., AND ScAssa, T. Intellectual property and the licensing of canadian government geospatial data: An examination of geo-connections' recommendations for best practices and template licences. The Canadian Geographer/Le Geographe Canadien 54, 3 (2010), 366-374. 
[57] Kahneman, D. Thinking, Fast and Slow. Macmillan, 2011.

[58] Kahneman, D., Sibony, O., And Sunstein, C. R. Noise: A Flaw in Human Judgment. Little, Brown, 2021.

[59] Kirkpatrick, K. Stage 1 and stage 2 of light rail transit (lrt) - provision of public washrooms. Finance and Economic Development Committee November (2015).

[60] Klatzky, R. L., and Lederman, S. J. Representing spatial location and layout from sparse kinesthetic contacts. Journal of Experimental Psychology: Human Perception and Performance 29, 2 (2003), 310.

[61] Kobben, B., Huisman, O., and Lin, H. H. Combining vgi with viewsheds for photo tag suggestion. In Advances in Location-Based Services. Springer, 2012, pp. 181-190.

[62] KRAAK, M.-J. The role of the map in a web-gis environment. Journal of Geographical Systems 6, 2 (2004), 83-93.

[63] Kramer, G. An introduction to auditory display. Auditory DisplaySonification, Audification and Auditory Interfaces (1994), 1-77.

[64] Kristoffersen, S., And Lunngberg, F. Designing interaction styles for a mobile use context. In International Symposium on Handheld and Ubiquitous Computing (1999), Springer, pp. 281-288.

[65] Kristoffersen, S., And Ljungberg, F. "making place" to make it work: Empirical explorations of hci for mobile cscw. In Proceedings of the International ACM SIGGROUP Conference on Supporting Group Work (1999), pp. 276-285.

[66] Krug, S. Rocket Surgery Made Easy: The Do-It-Yourself Guide to Finding and Fixing Usability Problems. New Riders, 2009.

[67] KuHn, W. Volunteered geographic information and giscience. NCGIA, UC Santa Barbara (2007), 13-14.

[68] Liu, S. B., And Palen, L. The new cartographers: Crisis map mashups and the emergence of neogeographic practice. Cartography and Geographic Information Science 37, 1 (2010), 69-90.

[69] Lokuge, I., And Ishizaki, S. Geospace: An interactive visualization system for exploring complex information spaces. In Proceedings of the SIGCHI Conference on Human Factors in Computing Systems (1995), pp. 409-414.

[70] Loomis, J. M., Golledge, R. G., And Klatzky, R. L. Gps-based navigation systems for the visually impaired. Fundamentals of Wearable Computers and Augmented Reality 429 (2001), 46. 
[71] MacEachren, A. M. How Maps Work: Representation, Visualization, and Design. Guilford Press, 2004.

[72] Martinez, E., And Reyes, M. D. C. Cybercartography and society. In Modern Cartography Series, vol. 4. Elsevier, 2005, pp. 99-121.

[73] Meenl, P. E. Clinical versus statistical prediction: A theoretical analysis and a review of the evidence.

[74] Millar, S. Spatial memory by blind and sighted children. British Journal of Psychology 66, 4 (1975), 449-459.

[75] Millar, S. Memory in touch. Psicothema 11, 4 (1999), 747-767.

[76] Moellering, H. Analytical cartography. American Congress on Surveying and Mapping.

[77] Moellering, H. The nature of analytical cartography: An introduction. Cartography and Geographic Information Science 27, 3 (2000), 187-188.

[78] Oakley, I., McGee, M. R., Brewster, S., And Gray, P. Putting the feel in 'look and feel'. In Proceedings of the SIGCHI conference on Human Factors in Computing Systems (2000), pp. 415-422.

[79] OBJ STAFF. Oc transpo changing bus service at blair, tunney's pasture to ease crowded stations. Ottawa Business Journal (2019).

[80] OC Transpo. Vehicle accessibility features. Transit Accessibility 1 (2021).

[81] O’Hara, M. T., Watson, R. T., and Kavan, C. B. Managing the three levels of change. Information Systems Management 16 (1999), 63-70.

[82] O'NeIL, C. Weapons of Math Destruction: How Big Data Increases Inequality and Threatens Democracy. Crown, 2016.

[83] Oviatt, S. Taming recognition errors with a multimodal interface. Communications of the ACM 43, 9 (2000), 45-51.

[84] Oviatt, S., Cohen, P., Wu, L., Duncan, L., Suhm, B., Bers, J., Holzman, T., Winograd, T., Landay, J., Larson, J., Et al. Designing the user interface for multimodal speech and pen-based gesture applications: Stateof-the-art systems and future research directions. Human-Computer Interaction 15,4 (2000), 263-322.

[85] Oviatt, S., DeAngeli, A., And Kuhn, K. Integration and synchronization of input modes during multimodal human-computer interaction. In Proceedings of the ACM SIGCHI Conference on Human Factors in Computing Systems (1997), pp. 415-422. 
[86] Pascoe, J., Ryan, N., And Morse, D. Using while moving: Hci issues in fieldwork environments. ACM Transactions on Computer-Human Interaction (TOCHI) 7, 3 (2000), 417-437.

[87] Phillips, R. J. Making maps easy to read - a summary of research. In Processing of Visible Language. Springer, 1979, pp. 165-174.

[88] Piccoli, G., And Pigni, F. Information Systems for Managers: With Cases. Prospect Press, 2019.

[89] Pringle, J. Ottawa ride-sharing companies agree to hike accessibility fee to 10 cents per ride. CTV Ottawa 1 (2021).

[90] Pulsifer, P. L., Laidler, G. J., Taylor, D. F., And Hayes, A. Towards an indigenist data management program: Reflections on experiences developing an atlas of sea ice knowledge and use. The Canadian Geographer/Le Geographe Canadien 55, 1 (2011), 108-124.

[91] Reyes, C., Fraser Taylor, D., Martinez, E., and Caloca, F. L. Geo-cybernetics: A new avenue of research in geomatics? Cartographica: The International Journal for Geographic Information and Geovisualization 41, 1 (2006), 7-20.

[92] Richardson, A. Gottago! making progress in ottawa. Environmental Sustainability October (2015).

[93] RoccA, R. Coronavirus: 1st case of covid-19 reported in ottawa.

[94] Roth, R. E. Interactive maps: What we know and what we need to know. Journal of Spatial Information Science 2013, 6 (2013), 59-115.

[95] SAFFer, D. Designing Gestural Interfaces: Touchscreens and Interactive Devices." O'Reilly Media, Inc.", 2008.

[96] Scassa, T. Journalistic purposes and private sector data protection legislation: Blogs, tweets and information maps. Queen's LJ 35 (2009), 733.

[97] Scassa, T. Geographic information as personal information.

[98] Scassa, T. Legal issues with volunteered geographic information. The Canadian Geographer/Le Geographe Canadien 5\%, 1 (2013), 1-10.

[99] Scassa, T., And Deturbide, M. E. Electronic Commerce and Internet Law in Canada. CCH Canadian Limited, 2004.

[100] ShI, Y., AND PAI, D. K. Haptic display of visual images. In Proceedings of IEEE 1997 Annual International Symposium on Virtual Reality (1997), IEEE, pp. 188-191. 
[101] Statistics Canada. Low income measure (lim) thresholds by income source and household size.

[102] Stigler, S. M. Gauss and the invention of least squares. The Annals of Statistics (1981), 465-474.

[103] Stohr, W. B., And TAylor, F. Development from above or below? the dialectics of regional planning in developing countries.

[104] Sui, D., And Goodchild, M. The convergence of gis and social media: Challenges for giscience. International Journal of Geographical Information Science 25, 11 (2011), 1737-1748.

[105] TAYLOR, D. A conceptual basis for cartography: New directions for the information era. The Cartographic Journal 28, 2 (1991), 213-216.

[106] TAYlor, D. Cartography for knowledge, action and development: Retrospective and prospective. The Cartographic Journal 31, 1 (1994), 52-55.

[107] TAYlor, D. F. Maps and mapping in the information era. In Proceedings of the 18th ICA/ACI International Cartographic Conference, Stockholm, Sweden (1997), pp. 23-27.

[108] TAYlor, D. F. The concept of cybercartography. In Maps and the Internet. Elsevier, 2003, pp. 405-420.

[109] TAYLOR, D. F. Some recent developments in the theory and practice of cybercartography: Applications in indigenous mapping: An introduction. In Modern Cartography Series, vol. 5. Elsevier, 2014, pp. 1-15.

[110] Taylor, D. F., Anonby, E., And Murasugi, K. Further Developments in the Theory and Practice of Cybercartography: International Dimensions and Language Mapping. Elsevier, 2019.

[111] Taylor, D. F., And Lauriault, T. Cybercartography: Theory and Practice. Elsevier, 2006.

[112] Taylor, F., And Mackenzie, F. Development from within: Survival in rural africa.

[113] Terken, J., AND Verhelst, L. Information presentation for a wearable messenger device. In International Conference on Multimodal Interfaces (2000), Springer, pp. 542-548.

[114] Thaler, R. H., And Sunstein, C. R. Nudge: Improving Decisions About Health, Wealth, and Happiness. Penguin, 2009. 
[115] Tidwell, J. Designing Interfaces: Patterns for Effective Interaction Design. " O'Reilly Media, Inc.", 2010.

[116] Tkaczevski, A. Auditory interface problems and solutions for commercial multimedia products. Georgia Institute of Technology.

[117] ToBler, W. The development of analytical cartography: A personal note. Cartography and Geographic Information Science 27, 3 (2000), 189-194.

[118] Tsou, M.-H. Revisiting web cartography in the united states: The rise of user-centered design. Cartography and Geographic Information Science 38, 3 (2011), 250-257.

[119] Tumilty, R. Computer failure caused thursday's lrt delay. CBC (2019).

[120] Turner, A. Introduction to Neogeography. " O’Reilly Media, Inc.", 2006.

[121] TwitterDev. Building high-quality filters for getting twitter data. Developer Platform Twitter Inc. (2021).

[122] VAVER, D. Intellectual property law: Copyright, patents, trade-marks.

[123] Vul, E., And Pashler, H. Measuring the crowd within: Probabilistic representations within individuals. Psychological Science 19, 7 (2008), 645-647.

[124] Walker, J. Human Transit: How Clearer Thinking About Public Transit can Enrich our Communities and our Lives. Island Press, 2012.

[125] Wheless, G. H., Lascara, C. M., Valle-Levinson, A., Brutzman, D. P., Sherman, W., Hibbard, W. L., and Paul, B. E. Virtual chesapeake bay: Interacting with a coupled physical/biological model. IEEE Computer Graphics and Applications 16, 4 (1996), 52-57.

[126] Williamson, C., And Shneiderman, B. The dynamic homefinder: Evaluating dynamic queries in a real-estate information exploration system. In Proceedings of the 15th annual international ACM SIGIR conference on Research and development in information retrieval (1992), pp. 338-346.

[127] Wu, T. The Master Switch: The Rise and Fall of Information Empires. Vintage, 2010.

[128] Zielstra, D., AND Zipf, A. A comparative study of proprietary geodata and volunteered geographic information for germany. In 13th AGILE International Conference on Geographic Information Science (2010), vol. 2010.

[129] Zuboff, S. The Age of Surveillance Capitalism: The Fight for a Human Future at the New Frontier of Power. Profile books, 2019. 
Appendices 


\section{Appendix A}

\section{Transit Commission}

MEMBERSHIP

Membership of the Transit Commission shall consist of eight (8) Members of Council and four (4) citizen members, as approved by Council. The Mayor is an ex-officio member of the Commission. The Chair and Vice-Chair shall be appointed by the membership of the Commission from among the members of the Commission who are also Members of Council.

Where Joint Committee meetings of the Transit Commission and another Committee of Council are held to consider matters of which transit is a component, citizen members of the Commission shall be non-voting, ex-officio members of the Joint Committee.

\section{MATTERS FOR WHICH THE COMMISSION IS RESPONSIBLE TO COUNCIL}

The Transit Commission shall:

1. Be responsible to Council for those items related to the operation of transit services emanating from:

(a) The applicable services areas and branches of the Transportation Services Department;

(b) Advisory Committees or Sub-committees that are within the area of responsibility of the Transit Commission; and

(c) Local boards, agencies and corporations that are created by, and report to, this Commission.

2. Subsequent to the approval of the budget, consider, for recommendation to Council, all operating and capital budget adjustments pertaining to items within the Commission's mandate and in excess of the overall Council-approved transit budget.

3. Review and recommend to Council transit fares, the annual operating and capital budget for the Transportation Services Department, excluding those under the mandate of other Standing Committees.

4. Ensure co-ordination and consultation with Standing Committees and departments where responsibilities overlap on transit matters and on issues relevant to the mandate of more than one Committee. 
5. Consult with the Agriculture and Rural Affairs Committee and recommend to Council any changes that would affect taxation in the rural transit areas.

6. Review and recommend to Council revisions to the Transit Commission Terms of Reference, as required.

7. Recommend to Council, the City of Ottawa's participation in federal or provincial cost-sharing programs for matters within the mandate of the Commission.

8. Recommend to Council proposed by-laws that are under the Commission's jurisdiction.

9. Review and recommend to Council strategic plans strictly related to transit matters.

10. Review and make recommendations to Council on City policies and plans that have a public transit component.

11. In collaboration with the Transportation Committee, review and make recommendations to Council on transit infrastructure matters, such as rapid transit corridors and traffic management, so as to achieve the transit goals of the City's Official Plan and the Transportation Master Plan.

RESPONSIBILITIES UNDER DELEGATED AUTHORITY OF THE COMMISSION

The Transit Commission shall have final decision-making authority with respect to the following specific responsibilities:

1. Provide direction on, and ensure the implementation of, policies and programs with respect to the operation of Transit Services.

2. Exercise other specific responsibilities set forth by relevant statutes and City Council.

3. Consider and approve all operating and capital budget adjustments pertaining to items within the Commission's mandate and within the overall Councilapproved transit budget.

4. Pursue the following objectives in order to provide the best possible public transportation service for all transit customers:

(a) Attract an increasing number of customers to transit customers;

(b) Determine and meet the transit needs of residents and visitors;

(c) Provide excellent customer service and foster a reputation of safety and reliability; 
(d) Provide a transit service that is accessible and affordable;

(e) Ensure that safety standards are managed in accordance with recognized public transportation standards;

(f) Strive to develop and maintain a system that is adaptable to emerging changes in technology; and,

(g) Support policies that are consistent with the City of Ottawa Official Plan objectives.

5. Identify new transit services by-laws, policies, guidelines, and government funding programs for transit operations.

6. Act as the official bargaining agent of the City of Ottawa in negotiations with representatives of the Transit Services' unions representing OC Transpo workers within Council-approved mandates. The Commission may delegate its bargaining powers, however, the Commission has the authority to direct staff on the parameters of the negotiations and no final agreement resulting from such a delegation shall be binding until it is approved by the Commission and Council.

7. Review and approve all changes to transit network policy and performance standards related to operations.

8. Review and determine the service level standards for and the allocation of budgeted resources among urban and rural transit taxation areas.

9. Receive and approve staff recommendations for major service changes.

10. Receive and approve Transit wayfinding, branding and advertising standards and alternative revenue strategies for the transit system.

11. Provide direction on strategic asset plans (Fleet composition, stations, Park and Ride, on-street amenities) related to operations.

12. Review and approve technology matters related to public transit operations, including Smart Card, on-board technology, communication, control and monitoring systems, real-time data, and customer information, with the ability to refer technology matters to the Information Technology Sub-committee for input as the Transit Commission deems necessary.

13. Receive operational performance reporting related to the transit system.

14. Hear briefings on litigious matters related to the operation of transit services and to give direction to the City Solicitor on such matters where it is consistent with current Council direction or policy.

15. Receive reports from staff regarding the exercise of delegated authority (By-law 2019-280) on items within the Commission's mandate. 
16. Receive regular status updates regarding inquiries and motions relating to matters under the Commission's mandate.

17. Receive delegations from the public and hold public hearings as required by statute and Council.

18. Have the delegated authority to decide matters that are consistent with the application of federal and/or provincial statutes and/or regulations.

19. Pursuant with the Delegation of Authority By-law (Section 10 - Conferences and Conventions), approve Members' travel and attendance at conferences that are related to the Commission's mandate (e.g. Canadian Urban Transit Association (CUTA)).

20. Provide direction and guidance with respect to the operation of interprovincial public transit and co-ordination with the Société de transport de l'Outaouais ("STO").

21. Receive any audits within the Commission's mandate.

Last accessed on March 17 2021. https : //ottawa.ca/en/city-hall/your-city-government/ council-and-standing-committees\#transit-commissionTransitCommission 


\section{Appendix B}

\section{Cybercartography: A Multimodal Approach}

\section{B.0.1 Speech and Cartographic Visualization}

The inclusion of speech in the design of online interactive mapping systems has the potential to enhance the visualization of cartographic information. The speech modality allows the hands and eyes the freedom to perform other tasks, while the map user's auditory system is actively engaged with content in a cybercartographic atlas [110]. Speech is optimal for the description of events, objects, hidden objects, conjoint information, and for conveying time status [83, 31]. The description of dynamic events, objects and conjoint information can be commonplace in geographic visualizations that include temporal-spatially organized information (e.g., voice narration of a visual representation). Alternatively, speech is not good modality for the conveyance of visual display navigation commands such as 'up', 'down', 'left', and 'right'. These commands would likely need to be repeated several times to adjust a map display to a desired state. For the conveyance of visual navigation commands, a mouse, cursor key or custom pointing device is better suited for the task [110].

The design of voice-guided interfaces should accommodate variance in diction and enunciation, but allow for differentiation between similar sounding words. The map user and the mapping system should have the same orientation perspective, to manage the map user's cognitive workload. In general, the effectiveness of voice-based commands is dependent upon:

1. Provision of audible and comprehensible synthetic voice output;

2. Appropriate timing at which the information is presented; and,

3. Appropriate amount of information delivered to the user (i.e., promote a state of flow, without triggering information overload) [110].

Terken and Verhelst (2000) evaluated information retention using a wearable device that presented a user with information as text, voice, or a combination of text and voice. They found that even if full synchronization of the speech and text cannot be achieved, combined presentation of speech and text is superior than either alone [113]. We interpret these findings to suggest that some information conveyed bi-modally, between a map user and a mapping system, promotes greater communication and interactivity, relative to information communicated by either modality alone.

Cognitive workload implications play an important role in the determination of which psychological factors should be considered when integrating speech into cartographic visualizations [110]. A map user's ability to preform certain tasks can be 
affected by cognitive workload, even when the workload is spread across modalities. When designing a mapping system, the map-maker should account for cognitive interference that can present when a map user performs concurrent tasks that require the same processing resources (e.g., performing concurrent visual searches for unrelated items, or receiving concurrent voice instructions identifying a directional change and unrelated route detour).

Working memory is conceptualized as a cognitive system, with a limited capacity, that can hold information in a temporary state. Baddeley (1981) proposed that working memory consists of a central executive, a phonological loop, and a Visual-Spatial Scratch Pad (VSSP) [6]. He describes the central executive as the coordinator of the phonological loop and the VSSP, and notes that the phonological loop is responsible for mental rehearsal and is involved in the temporary storage of verbal information, while the VSSP acts as a mental blackboard and serves as a temporary storage for visual and spatial information [7]. Competing demands for the same cognitive resource can lead to decrements in performance. Multimodal interfaces can help manage information overload by spreading the cognitive workload across multiple modalities [52]. The bi-directional use of the speech modality in cartographic visualizations allows the map designer and map user to communicate more efficiently, while simultaneously managing the increased cognitive workload across multiple modalities [110].

\section{B.0.2 Gesture and Cartographic Visualization}

Gesture interactions are defined as user initiated body movements, such as pointing or touching with the finger, or the use of a specialized instrument (e.g., a stylus) to interact with a user interface. Gestures have the potential to facilitate user interaction with dynamic computer maps, and may accelerate the visualization of geographic information [110]. Although gesture interactions feel natural for many navigation and direct manipulation tasks, they may be inappropriate for tasks that require precision [12]. Moreover, gesture or pen-based interactions can provide a map user with greater privacy in public settings, and offer an effective alternative when voice interaction is not practical due to environmental conditions. Pen interactions can be highly effective in mobile and field operations, as they may provide users with the added the flexibility to draw symbols, signs, digits, etc., while conducting field observations [110].

At present, the stylus is compatible with most smartphones and tablets, and is commonly used for handwriting and gestural input. Mobile-based mapping systems support several visual-spatial applications including: navigation and sketching, defining spatial relations, and the use of precise points and lines to define spatiallyreferenced information [110]. The use of hand-drawn graphics in computer generated mapping systems has risen steadily over the past decade. Numerous gesture-based interactions are now commonplace in a wide variety of mapping environments, from in-game analysis provided by sports commentators to real time election coverage, the stylus is used in combination with finger gestures to add information overlays, and additionally to interact with cartographic visualizations generated by computer-based 
mapping systems [110].

Much of the early work that considered multimodal interactions in graphical applications focused on evaluating the potential for speech and gesture interactions as a communication tool $[12,30,85]$. When considered together, speech and gesture interactions enable a user to describe events, spatial layouts, objects, and their relationship to one another in ways that a physical mouse and keyboard cannot. The strengths of speech and gesture interactions are complimentary; a careful examination of the relative roles of speech and gesture during human conversation reveals that speech is most often used to convey the subject, verb and object, while gesture is used to describe the relative location of things [85].

Hauptmann and McAvinney (1993) evaluated a speech and gesture recognition system by observing participant interactions with graphical applications across three conditions: speech only, gesture only, or a combination of speech and gesture. They observed a surprising degree of commonality in the use of gestures and speech, and noted a preference among participants for the combined gesture/speech interactions, especially when for spatial tasks [50]. Their findings suggest that users prefer multimodal interaction over unimodal speech or gesture interaction while performing spatial tasks in graphical applications.

When considering the inclusion of gestural interaction in graphical applications, the interface designer should be cognisant of the limitations of working memory. Dan Saffer (2008) suggests that an increase in user difficulty should be expected as the number of unique gestures in an application increases. Hence, the observed benefits of gesture interactions, namely the added portability and flexibility they provide, begin to plateau when the cognitive workload exceeds the capacity of working memory and the user becomes unable to distinguish the functions attributed to several unique gesture interactions [95]. Again, multimodal interfaces have been shown reduce the peak cognitive workload by spreading it across multiple modalities [85].

\section{B.0.3 Sound and Cartographic Visualization}

The use of sound in the design of online interactive maps can enhance cartographic visualizations when it is used to distribute the cognitive workload between the visual and auditory modalities. Researchers have developed mapping applications that use sonic variables such as pitch, duration, loudness, silence and modulation to form sonic overlays that enable concurrent and integral visualization of predominantly visual representations $[34,39,53]$. A classification of visual information, for example, a visual symbol paired with an auditory tone that enables the user to act on an audible cue without engaging the visual modality has been shown to reduce cognitive workload for the visual modality. The auditory modality creates a third dimension for the communication and visualization of cartographic information traditionally displayed on two-dimensional maps [110].

Researchers have suggested that abstract sounds (i.e., non-speech sounds) can be used to improve the performance and usability of human-computer interfaces $[8,13]$. 
Barrass and Kramer (1999) found that synthetic non-verbal sounds can represent numerical data and provide support for information processing activities. Brewster (1994) described the abstract sounds as structured audio messages called earcons. Earcons are abstract synthetic tones that can be used in structured combinations to create sound messages which represent different the parts of a mapping interface [17]. Sound messages typically vary in duration, location, loudness, pitch, timbre, and rhythm. By varying the complexity and combinations of sound elements, hierarchical earcons can be created to represent hierarchical map data structures [13].

A benefit of incorporating sound in the design of human-computer interfaces is its ability to replace text on small screens, such as cell phones or in-vehicle infotainment systems, thereby decreasing visual clutter and increasing screen real estate $[65,86]$. An auditory stimulus can also be used to convey information when the user's visual system is engaged in a resource intensive task, such as driving. Information conveyed via an auditory stimulus can be delivered regardless of the user's visual behaviour [64]. Moreover, sound is attention grabbing, while a user can choose to not look at something; it is significantly more difficult for a user to avoid hearing something. Thus, an auditory stimulus should be considered when important information must communicated by the mapping system [8].

One should also consider the limitations associated with use of sound in cartographic visualizations. Kramer (1994) highlighted some difficulties a user might encounter when presented with an auditory stimulus: he found that only a few different values can be used to represent volume unambiguously, whereas visual representations have a fine gradation of values [63]. It is difficult to present absolute data when deploying sonication, as a user's perceptual acuity is much less capable, relative to the capacity of the visual modality. Users with perfect vision can look at a value on a graph to get an absolute value, while sound disappears beyond the phonological loop and a user must remember the information that the sound represented [110]. Information that is displayed visually allows the user to review it as necessary while carrying out a task.

A limitation associated with the use of earcons is 'learnability' $[70,116]$. New users of earcons are typically able to learn between 4 and 6 symbolic sounds in a matter of minutes. However, mastery of 10 or more earcons is difficult for many people and not possible for some. Tkaczevski (1996) illustrated the limitations of earcons in his research about the use of sonication in the design of a nuclear power plant control system. He found that the use of earcons led to an improvement in reaction time, diagnostic accuracy and correct response to alarms; however, he noted that few non-audio workers had the vocabulary of terms required to describe what was needed to create a rich computer experience in the auditory realm, and therefore, were reluctant to further explore the diagnostic capabilities of sound [116].

Wheless et al. (1996) presented an optimal combination of visual and sound modalities in the design of a virtual reality representation of the Chesapeake Bay ecosystem. They used sound to represent salinity levels, where a change in salinity was denoted by a change in pitch. When visualizing the Bay's topography, a user 
could listen to changes in salinity, and the user was able to form an understanding of the linkage between the Bay's biological and physical systems [125]. Employing the auditory modality to convey additional information while a user's visual modality is concurrently engaged can reduce the cognitive workload implications associated with the visual modality.

\section{B.0.4 Haptic and Cartographic Visualization}

The use of haptic (both tactual and kinaesthetic) information to represent geographic phenomena has been given limited attention as a method for exploring data, due to the difficulty of implementing tactual and kinaesthetic methods [45]. A few studies have explored the use of non-visual modalities to represent information for blind and visually impaired people, but there is limited research that explores tactile languages for fully sighted map-users. Vasconcellos (1991) formulated a set of haptic variables by translating Bertin's (1983) visual variables into tactile variables, and tested the tactile language with children and school teachers in Brazil. However, she did not develop any tactile variables that did not have an equal visual counterpart. Virtual reality is a promising technology that may be deployed to represent spatial relations using haptic representations, using specialized devices such like gloves, computer mice and joysticks that offer additional capacity for precise kinaesthetic sensation [100].

Bosman et al. (2003) explored the potential for haptic output to deliver guidance for pedestrians who do not self-identify as having any specific disability to find their way to a particular destination indoors, e.g., a room in a building [14]. In their study, participants were asked to locate target rooms in two conditions: either by using the visual wayfinding signage in the building, or by using their GentleGuide prototype with tactual feedback in the absence of wayfinding signage. All participants located the target rooms under both conditions; however, participants were able to navigate to the target rooms with less errors, which translates to boost in wayfinding performance, when they received tactual feedback from the prototype.

Oakley et al. (2000) used a haptic interface device to supplement traditional user interactions with graphic interfaces. First, they compared the effects of four different haptic augmentations on usability in a simple targeting task, and then had participants perform a more ecologically-oriented searching and scrolling task. They found that haptic effects did not improve user performance in terms of task completion time, although they reported a reduction in user error rates and subjective cognitive workload, when the combined haptic-visual interface was used [78]. Thus, this research demonstrates that haptic devices can provide significant performance benefits in a well-designed multimodal application.

Although there has been considerable research on the cognitive workload implications of visual and auditory interfaces, to date, there has been little research regarding haptic only interfaces [60]. Research on the cognitive processing of haptic stimuli is complicated due to haptic representations emanating in the presence of visual and phonological representations. Given the limits of working memory, there is a finite 
number of haptic representations than can be retained. Miller $(1975,1999)$ demonstrated that a maximum of three haptic representations can be retained simultaneously in working memory $[74,75]$.

When considering the use of haptic interfaces in cartographic visualizations, it is important to consider the potential risks associated with cognitive overload [45]. Cartographers have a historical familiarity with this issue, dealing with primarily visual interfaces for most of history. The cognitive workload associated with an overstimulating visual display is often overlooked because users do not have to retain all of the information in working memory. However, user distraction and a sense of helplessness does not serve to advance most spatial tasks. Conversely, auditory and haptic information must be retained in working memory, unless digitally preserved and accessible via the interface, and may contribute to an unintended effect, an increase in the users cognitive workload [45]. 


\section{Appendix C}

\section{The Inuit Siku (Sea Ice) Atlas}

The Inuit Siku Atlas was developed as an educational resource to share knowledge, stories, maps, and language artefacts from years of interviews, focus groups, sea ice commutes and workshops with sea ice experts in Cape Dorset, Iglookik, Pangnirtung, and Clyde River Nunavut [110]. The atlas is hosted online, and features multimedia content intended for local students and the general public who wish to explore and learn about various sea ice topics, from Inuit perspectives. The development of the atlas was guided by Inuit elders' and hunters' expressions of interest to:

- Share their knowledge with local youth;

- Develop more Inuit knowledge content in the local education system; and

- Share Inuit knowledge more broadly with scientists and non Inuit [110].

The Inuit Siku Atlas is an educational resource, comprised of Inuit sea ice knowledge, documented by local community and university researchers between 2004-2008. The central artefacts in the atlas are:

- Inuit knowledge of sea ice features, uses, hazards, and changes over time;

- Traditional Knowledge from community elders, hunters, and local researchers involved in the project;

- maps of Inuit sea ice knowledge and uses;

- Inuktitut sea ice terminology for different seasons;

- the Floe Edge Service - satellite imagery available for communities across the Canadian North; and

- background information on the larger sea ice project and researchers involved [110].

Educators, students and community members provided important feedback during informal community consultations and workshops. Community consultations included the deployment of over 90 surveys, one third of which were fully completed, and two thirds were partly completed. All ages and many occupations were represented across the surveys [110]. Overall, the feedback was very positive, with $57 \%$ of respondents indicating that they liked the atlas very much and felt that the content was highly relevant and useful, and $31 \%$ of respondents indicating that they liked the atlas and felt it was highly relevant and useful [110]. The main positive comments the atlas creators received included the fact that respondents: 
- Liked having the knowledge available and accessible for educational purposes;

- Appreciated the public sharing of research results;

- Could identify the contributions t language preservation;

- Found the atlas content to be engaging for students;

- Enjoyed the highlighted contributions and background from elders;

- Were appreciative that the atlas design permitted Inuit voices to be heard directly (through audio/visual modalities); and

- Expressed an overall satisfaction about the compilation of sea ice maps and the terminology used [110].

A number of concerns were also raised; however, many of these concerns could not be adequately addressed prior to the launch of the Inuit Siku Atlas. The practice of Cybercartography is iterative, and work to address many of the concerns identified by the community remains ongoing [110]. Some of ongoing work includes:

- Ongoing issues of limited bandwidth across the North;

- Accessibility/use of reporting tools by Elders, hunters, or those without computers or who are unilingual in Inuktitut;

- Interest in having additional communities represented;

- Language barriers between researchers and the local community, and also intercommunity language barriers;

- An interest in having the site bilingual throughout; and

- The ability for community members to update information themselves [110].

Additional feedback was also sought specifically from Inuit Elders and youth in each of the key participating communities. Several local Elder/youth workshops were hosted at community high schools, where Elders who contributed content to the Atlas were able interact with local youth who might use the Siku Atlas as an educational tool [110]. In all cases, students would navigate through the Atlas to show Elders different sections, and Elders would elaborate on the content contained in the Atlas and share their expansive and practical and knowledge about sea ice conditions with the students [110]. 


\section{Appendix D}

\section{The Arctic Bay Atlas}

The Arctic Bay Atlas began as a literacy research project, with a focus on documenting regional place names, in the early 2000s. The project was led by Nunavut Youth Consulting (NYC), a not for profit organization with a mandate to engage young people in positive community undertakings that addressed issues such as suicide and substance abuse [110]. Recognizing the learning potential of intergenerational research, the Nunavut Arctic College (NAC) supported NYC in initiating and guiding the Arctic Bay project in the collection and organization of data, in partnership with Elders and local youth. From 2003 to 2006, NYC worked in partnership with NAC, the Canada/Nunavut Geoscience Centre, and the Inuit Heritage Trust (IHT) on a literacy research project: the Traditional Name Placing Project. Local youth interviewed Elders and produced twelve maps with 350 traditional place names. In addition to the mapping of traditional geographical place names, some of the traditional trails that were used to travel between Arctic Bay and Igloolik were also documented. Through this work, NYC in partnership with Ron Elliot became aware of Claudio Aporta's work at the Geomatics and Cartographic Research Center (GCRC) at Carleton University, in Ottawa. Together, Aporta and NYC sought to develop a similar - but humbler - interactive atlas documenting traditional Inuit land skills, wayfinding, and related environmental knowledge in Igloolik, Nunavut [110].

In March 2006, NYC secured additional funding to extend their place naming documentation efforts with a project titled: 'Reclaiming the Land'. The youth driven project was, again, primarily focused on the Inuktitut Language, but was designed to build and continue intergenerational exploration, dialogue, and documentation of geospatial locations of cultural significance [110]. The process of active learning through identifying and mapping places of cultural importance continued to engage youth, and also attracted contributions from other community members such as hunters, who shared their experiences in different areas at different times of the year. In addition to the goal of preserving historical and traditional cultural land use, the project offered a dialogical learning experience with youth sharing their knowledge of contemporary technology, and Elders sharing their traditional geospatial knowledge [110].

Building on earlier iterations of the atlas, funding from the Heritage Canada Gateway Fund was secured through a joint effort from the GCRC research team, and IHT, NAC and NYC, in January 2007 [110]. The funding was used to support community-driven development of an interactive multimedia Arctic Bay Atlas website that presents Inuit culture, geographical locations and trails of importance, place names, oral history, and geographical representation to the Canadian public [110]. 
The project also sought to develop a local capacity in Arctic Bay to digitally capture and disseminate via the Internet, important Inuit knowledge, including how the community historically conceptualized and represented its identity in relation to space, time, land, and spiritual forces [109].

Over the course of atlas development, the resulting maps were not necessarily as rich and complex as hoped, but there remains great value in from the collaborative process [110]. The Arctic Bay Atlas is multi-authored, includes cross-generational sharing of knowledge, and fostered new partnerships between university researchers and the community [110]. Many significant learning objective were met in the production of the Arctic Bay Atlas:

1. The mentoring of youth in gathering and reflecting upon traditional Inuit knowledge;

2. Skills development in working with Elders and collecting oral narratives;

3. Stronger survival skills through knowledge of geography;

4. Enhanced language acquisition from learning traditional place names; and

5. Unique geographic and multimedia technical skills (including data management) [110].

The Arctic Bay Atlas was designed to increase the capacity of Nunavut research and education communities through interactive multimodal representations of their priorities, reflected in multiple perspectives, in time and space [110]. Developing community-based research practices adds capacity supports and is considered an appropriate and effective means of cross-cultural knowledge representation and sharing. It is the foundation for further understanding and development, and creates links between Inuit and Western scientific modes of researching and learning [110]. Central to the research process was the vision of Arctic Bay Youth in NYC who sought to engage with Elders and increase their understanding of traditional world-views and knowledge of land and language [110]. NAC continues to expand its learning program to support community-based knowledge building through research activities. New curriculum on research theory and practice, data management, and digital mapping research, combined with the experiences of local experts in atlas building will support communities in sharing and preserving their knowledge for generations to come [110]. 


\section{Appendix E}

\section{Semi-Structured Interview}

\section{E.1 Letter of Invitation}

Title: Development From Within: Am In-service Public Transit Narrative

Date of Ethics Clearance: February 202020

Ethics Clearance for the Collection of Data Expires: February 282021

Monday March 2 2020,

Dear Sir or Madam,

My name is Ryan Grenon and I am graduate student in the Master of Arts in Human-Computer Interaction program at Carleton University. I am working on a research project under the supervision of Dr. Fraser Taylor.

I am writing to you today to invite you to participate in a study that aims to utilize social media data to better understand the needs of persons who live with disabilities that limit their ability to interact with public transit infrastructure in Ottawa, Canada.

This study involves one 30-minute semi-structured interview and one 45-minute usability-testing session (you will be asked to use a piece of software on a laptop computer). Both sessions will take place in a mutually convenient, safe location (e.g. a coffee shop or public library). With your consent, the interview will be audio-recorded, and the usability-testing session will be audio and video recorded (audio-recording is a condition of participation in the study and video recording will be restricted in scope to recording your hands interacting with a computer mouse and keyboard only, i.e., no personally identifying information will be video recorded). Once the recordings have been transcribed, the audio and video recordings will be destroyed.

While this project can involve some professional and emotional risks in the event of a data breach of the primary investigator's personal computer and external hard drive, care will be taken to protect your identity. This will be done by keeping all responses anonymous and allowing you to request that certain responses not be included in the final project. Should you feel any distress following your participation in this project, you are invited to consult mental health resources in your community-please visit www.centretownchc.org http://www.carlington.ochc.org for additional information.

You will have the right to end your participation in the study at any time, for 
any reason during a session, and up to seven (7) days following your participation in the usability-testing session. If you choose to withdraw, all the information you have provided will be destroyed.

As a token of appreciation, I will be providing you with a Tim Horton's gift card valued at twenty $(\$ 20)$ dollars at the conclusion of the usability-testing session. There is no compensation if you choose not to participate in the usability-testing session.

All research data, including audio and video recordings and any digital notes will be password protected. Any hard copies of data (including any handwritten notes or USB keys) will be kept in a locked cabinet at Carleton University or in a locked cabinet in the researcher's private residence. Research data will only be accessible by the principal investigator (Ryan Grenon), the research supervisor (Dr. Fraser Taylor) and the graduate program supervisor (Dr. Robert Biddle). The ethics protocol for this project was reviewed by the Carleton University Research Ethics Board, which provided clearance to carry out the research. Should you have questions or concerns related to your involvement in this research, please contact:

CUREB-B:

If you have any ethical concerns with the study, please contact the Carleton University Research Ethics Board-B (by phone at 613-520-2600 ext. 4085 or via email at ethics@carleton.ca).

If you would like to participate in this research project, or have any questions, please contact the principal researcher via email at ryangrenon@cmail.carleton.ca or by phone at $1+(613) 8062217$.

Sincerely, Ryan Grenon 


\section{E.2 Screening Questionnaire}

Dear Sir or Madam,

Please read the four (4) statements listed below. If you agree with the statements (if they are true for you) please sign in the designated area.

1. I am fluent in the English language (written and oral communication).

2. I have personal experience using public transit infrastructure in the City of Ottawa within the past 12 months.

3. I am physically able to operate a computer mouse and keyboard (the mouse and keyboard are wireless and may be placed on a tray or other accessible surface).

4. I self-identify as an active Twitter user.

If the four statements above are true for you, please print and sign your name in the spaces below.

Participant signature:

Date: 


\section{E.3 Informed Consent Form}

Name and Contact Information of Researchers

Name: Ryan Grenon

Email: ryangrenon@cmail.carleton.ca

Project Supervisor: Dr. Fraser Taylor

\section{Project Title}

Development From Within: An In-Service Public Transit Narrative

\section{Carleton University Project Clearance}

Clearance \# 112088

Date of Clearance: February 202020

\section{Invitation}

You are invited to take part in this study because you identified as a person who is fluent in the English language, have utilized public transit infrastructure in Ottawa, Canada in the past 12 months, are physically able to operate a computer mouse and keyboard, and identify as an active Twitter user. The information in this form is intended to help you understand what we are asking of you so that you can decide whether you agree to participate in this study or not. Your participation in this study is voluntary, and a decision not to participate will not be used against you in any way. As you read this form, and decide whether to participate, please ask all the questions you might have, take whatever time you need, and consult with others as you wish.

\section{What is the purpose of the study?}

This study aims to use social media data to better understand the needs of persons who live with disabilities that limit their ability to use with public transit infrastructure in Ottawa, Canada.

\section{What will I be asked to do?}

If you agree to take part in the study, we will ask you to:

1. Participate in one semi-structured interview (30 minutes). The interview session will take place in a mutually convenient, safe location (e.g. a coffee shop or public library), and the session will be audio-recorded - this is a condition for participation in this study.

2. Participate in one usability-testing session, i.e., use a laptop computer to interact with a piece of software, and complete a post-test questionnaire (45 minutes)

\section{Risks and Inconveniences}

While this project can involve some professional and emotional risks in the event of a data breach of the primary investigator's personal computer and external hard drive, 
care will be taken to protect your identity. Should you feel any distress following your participation in this project, you are invited to consult mental health resources in your community-please visit www.centretownchc.org or http://www.carlington.ochc.org for additional information.

\section{Possible Benefits}

Your participation may allow researchers to better understand the needs of persons who live with disabilities that limit their ability to interact with public transit infrastructure in Ottawa, Canada. This may lead to improved policies that better address the needs of persons who live with disabilities that limit their ability to interact with public transit in Ottawa, Canada.

\section{Compensation/Incentives}

You will receive a "Thank You" card at the conclusion of the semi-structured interview, and you will receive a "Tim Horton's" gift card valued at twenty (\$20) dollars at the conclusion of the usability-testing session.

\section{No waiver of your rights}

By signing this form, you are not waiving any rights or releasing the researchers from any liability.

\section{Withdrawing from the study}

If you withdraw your consent during the course of the study, all information collected from you before your withdrawal will be discarded. After the study, you may request that your data be removed from the study and deleted by sending an email to the Principal Investigator (ryangrenon@cmail.carleton.ca) within seven (7) days of participation in the usability-testing session.

\section{Confidentiality}

We will treat your personal information as confidential, although absolute privacy cannot be guaranteed. No information that discloses your identity will be released or published without your specific consent. Research records may be accessed by the Carleton University Research Ethics Board in order to ensure continuing ethics compliance. The results of this study may be published or presented at an academic conference or meeting, but the data will be presented so that it will not be possible to identify any participants unless you give your expressed consent. You will be assigned a code so that your identity will not be directly associated with the data you have provided. All data, including coded information, will be kept in an encrypted file on a secure computer.

\section{Data Retention}


After the study is completed, your de-identified data will be retained for future research use.

\section{New Information during the study}

In the event that any changes to the study occur you will be promptly informed.

\section{Ethics Review}

This project was reviewed and cleared by the Carleton University Research Ethics Board B. If you have any ethical concerns with the study, please contact Carleton University Research Ethics Board (by phone at 613-520-2600 [ext. 4085] or by email at ethics@carleton.ca).

\section{Statement of consent}

I voluntarily agree to participate in this study. Yes or No

I agree to be audio/video recorded/photographed Yes or No

I agree to be contacted for follow up research Yes or No

Signature of Participant:

Date:

\section{Research team member who interacted with the participant}

I have explained the study to the participant and answered any and all of their questions. The participant appeared to understand and agree. I provided a copy of the consent form to the participant for their reference.

Signature of researcher:

Date: 


\section{E.4 Semi-Structured Interview Guide}

\section{Semi-Structured Interview Guide PG1}

PG1 = individual who self-identifies as a person living with a disability that limits their ability to use public transit infrastructure

\section{Background/understanding the participant as an individual}

- Name, age, occupation, hobbies?

- How would you describe your disability? Has your disability changed in the past 12 months? If yes, can you tell me a little bit about how it changed?

- How would you describe your mobility device? Have you modified, changed or upgraded your mobility device in the last 12 months? If yes, how so, and why?

- I want you to know that I am going to do my best to use appropriate language and not be offensive; if I do say something that offends you, I'd like you to stop me in the moment and let me know so that I don't offend you further-sound good?

\section{Understanding participant use of public transit infrastructure}

- Would you describe yourself as a conventional transit user, para transit user, or both? If both, can you tell me why you alternate between conventional and para service?

- ParaTranspo service - can you walk me through, step by step, the process of booking and using para transpo service?

- Is the first step making a reservation? Can you tell me a little bit about how that works? What is manageable, what is missing - does anything upset or annoy you about the current reservation process? I understand that you need to book your trip well in advance. Can you tell me a little bit about that?

- How would you describe the reliability of your five (5) most recent trips? Does your trip arrive on schedule? Why do you think that is the case? Do you notice any patterns, for example, is your ride late at certain hours of the day, does the weather have an impact on the reliability of para service?

- I understand that the City of Ottawa/OC Transpo has been sub-contracting para transit trips to accessible taxis because they are not able to meet service demand. Have you experienced this firsthand? Can you describe your experience for me? In your opinion, is this scenario (accessible taxi) a sustainable solution to address chronic under staffing of para transpo operators and buses at the City of Ottawa/OC Transpo? 
- Trip cancellations, you have made a reservation, and your trip is later cancelled? Does this actually happen? What is the explanation from the City of Ottawa/OC Transpo? What do you do if your trip is cancelled, what options do you have?

- I understand that para transit service is not available to the same degree as conventional bus service, in terms of hours of service. What are the hours of operation for para transit service? Do you have any experience with early morning or late-night para service? Do you think that you might stay out later or go out before sunset if the service was available? What do you do if you absolutely need a ride and the service is not operating at that time?

- \#paraparity, what does this mean to you? What is needed to achieve this goal?

- Is there anything else related to para transpo service that you would like to tell me about? What did we miss?

- You have identified some major challenges that para transit users face on a regular basis. In your opinion, what are the five (5) most urgent issues that need to be addressed?

1.

2 .

3 .

4 .

5.

- Conventional bus service - are you able to use it? Do you use it? If yes, tell me about how you make the decision to use conventional transit or para transit? What factors are involved in your decision making, for example, weather conditions, time of day, etc.

- If you were General Manager of transit in Ottawa - what is the first change you would implement? How would make it happen, can you explain what you think the process might look like? Why is this important to you?

\section{Understanding participant use of the social media platform, Twitter, to discuss public transit infrastructure}

- How would you describe your use of the social media platform "Twitter" to interact/converse with other twitter users about accessible transit infrastructure and para transit service, for example, would you say that you try to create awareness about public transit accessibility issues? How, please be as specific as you can, do you go about doing that, for example, do your tweets include text, photos, videos, hyperlinks to other web domains? 
- For each of the different medias you just mentioned, how do you think they impact the reach of your tweet? For example, do you think a tweet that includes text and a photo will receive more 'likes' and 'retweets', in other words, do you think adding additional media to a standard text tweet will increase your tweet's reach/exposure and gain a larger/wider audience? Do you think that including additional media to a 'text only' tweet can increase a reader's understanding of the message you are trying to convey?

- Do you use the \#hashtag function? Can you help me understand how you use this interaction technique? How do you decide what words get a hashtag and what words do not? Why? What are the 5 most active public transit \#hashtags right now?

1.

2 .

3.

4.

5.

- Do you use the @citizen function to target your tweets to a specific audience, e.g. a specific person, an advocacy group, or a politician? Do these people respond to your tweets? In terms of your recent twitter interactions, who (what people, organizations, media outlets, etc. have been responsive to your @them? Are you able to list 5 responsive @citizen right now?

1.

2 .

3 .

4 .

5.

- Do you feel like you are being heard on Twitter? How do you measure that?

- Do you live tweet, if yes, how often?

- Do you only tweet in a wifi area? Why, or why not?

- When you are at your private residence, do you tweet using your cell phone, a tablet, or a computer? A combination? Why do you choose to interact in this way?

- Do you use any third-party applications to manage your Twitter feed? If yes, can you name them for me please? 
- Why do you think you use the Twitter platform to communicate with others about public transit infrastructure? Why not use Facebook, why not write a blog?

- What other avenues exist for people to voice their concerns about accessible public transit infrastructure - outside of Twitter? Have you used any City of Ottawa/OC Transpo reporting tools? Just briefly, how did that go for you? What was the process like? Were your concerns addressed in a timely fashion, where they addressed at all?

- In your experience, what is the best avenue to resolve a concern related to accessible public transit infrastructure? Why do you think this is the case?

- When you bring your concerns to the decision-making table, how do you prepare your verbal statement (text, do you ever include images in your notes)? Would you like to see more options/modalities, like pre-recorded video messages or the ability to show photos or images on the projector screens at Ottawa City Hall, included as options for participating in the democratic process? Why?

- I will be speaking with some people involved in accessible public transit advocacy work next week. I have a list of some people that I would like to speak with, but I'd like to know if there is a person or grassroots community group in the accessible public transit advocacy space that you think I should speak with? What community groups are speaking up on behalf of people living with disabilities who use public transit right now?

1.

2 .

3.

4 .

5 .

- Thank you sharing your time with me today. The interview is now over, and I will end the audio recording at this time. 


\section{Semi-Structured Interview Guide PG2}

PG2 = individual affiliated with an NGO or non profit advocate/lobby group related to public transit

Background/understanding the participant as an individual

- Name, age, current occupation, hobbies outside of work?

- I want you to know that I am going to do my best to use appropriate language and not be offensive; if I do say something that offends you, I'd like you to stop me in the moment and let me know so that I don't offend you further - sound good?

\section{Understanding participant use of public transit infrastructure}

- How would you describe your use of the public transit system in Ottawa? Are you a conventional or para transit user, or both? Do you consider yourself a seasonal transit user, year-round user, occasional user, etc.?

- Do you ride on the new Confederation Line (the LRT)? If so, would you describe your usage as casual (less than once per week) or regular (more than once per week)?

\section{Understanding participant use of the social media platform, Twitter, to discuss public transit infrastructure}

- In your own words, how would you describe your use of the social media platform, Twitter, to interact/converse with other Twitter users about issues related to accessible public transit infrastructure, both conventional and para service? For example, do you use Twitter to raise awareness about issues related to public transit accessibility? How, please be as specific as you can, do you go about doing that? Do you include different forms of media (e.g. photos/images, audio/video, links to other media) in your tweets?

- For each of the different forms of media that you just mentioned, how do you think each individual form of media impacts the reach of your Tweet? For example, do you think that a Tweet that includes photos or audio/visual content, in addition to text, will generate more "views", "likes" or "retweets"? Why?

- As an advocate/lobbyist for accessible public transit, I imagine that sometimes you might need to 'find out' what the issues are (maybe there are some issues that, as a non para transit user, you may never encounter), how would you go about doing that?

- - Do you use the @function to tag an audience? If so, can you tell me a little bit about why you do this? And who do you tend to tag? E.g., individual accounts, broadcast media outlets, NGO's, community groups, your team? 
- Off the top of your head, can you name a few Twitter accounts that are active in the accessible public transit conversation on Twitter right now (conventional or para service)?

1.

2 .

3 .

4 .

5.

- Do you use the \#hashtag function to participate in conversations about trending topics related to accessible public transit (e.g. \#ParaParadis, \#OCTranspo, \#ParaTranspo \#PWD)?

- Off the top of your head, can you name a few active \#hashtags in the accessible public transit conversation on Twitter right now (conventional or para service)?

1.

2 .

3.

4 .

5.

- What are the main issues being raised on Twitter, as you understand them, by para transit users and their activists/lobbyists right now?

1 .

2 .

3 .

4.

5.

- If you were to advocate/lobby in an organized setting, say you request to speak directly to the public transit decision-making table, i.e., Transit Commission, or in other organized settings like a town hall, how do you present your statements/motions/arguments? I imagine that you prepare some notes ahead of time, have you ever referenced active social media conversations at the decisionmaking table?

- Do you have the ability to introduce audio/video material as a knowledge generating tool, say in addition to a prepared statement/motion that is being discussed at the decision-making table? 
- What is the most challenging aspect about interacting with twitter users who constantly express their dismay about the state of the public transit system via Twitter (e.g., 280-character limit, lack of clarity about an issue, several mentions about the same topic, information overload)?

Thank you for sharing your time with me today. Before we part ways, would you like to hear a little bit about the nature of the project that we're working on? Great!

We are speaking with three groups of people for purpose this project. The three groups of people are:

1. People who self-identify as a person living with a disability who uses public transit

2. Representatives from community groups/NGOs who advocate/lobby on behalf of people who self-identify as a person living with a disability who uses public transit

3. Elected and Appointed officials who hold voting rights at the public transit decision-making table

So, what are we hoping to do with the information gathered from today's interview?

We are looking to harvest the active Twitter conversation about accessible public transit in Ottawa, with a deliberate focus on identifying the needs of the PWD community, as they are defined and discussed by the PWD community. To do this, we secured a Twitter Developer Account, which allows us to make use of the Twitter API (Application Programming Interface) to pull raw Twitter data, store it in our database, and then present this data to the aforementioned stakeholder groups in the form of an interactive atlas, that serves to empower and unite the PWD community, and better define the issues of greatest importance to the PWD community, for the public transit decision-makers. 


\section{Semi-Structured Interview Guide PG3}

PG3 = elected/appointed official who holds voting rights on the Transit Commission

\section{Background/understanding the participant as an individual}

- Name, age (approximation is fine), current occupation, hobbies outside of work?

- I want you to know that I am going to do my best to use appropriate language and not be offensive; if I do say something that offends you, I'd like you to stop me in the moment and let me know so that I don't offend you further-sound good?

\section{Understanding participant use of public transit infrastructure}

- How would you describe your use of the public transit system in Ottawa? Are you a conventional or para transit user, or both? Do you consider yourself a seasonal transit user, year-round user, occasional user, etc.?

- Do you ride on the new Confederation Line (the LRT)? If so, would you describe your usage as casual (less than once per week) or regular (more than once per week)?

\section{Understanding participant use of the social media platform, Twitter, to discuss public transit infrastructure}

- In your own words, how would you describe your use of the social media platform, Twitter, to interact/converse with other Twitter users about issues related to accessible public transit infrastructure, both conventional and para service? For example, do you use Twitter to raise awareness about issues related to public transit accessibility? How, please be as specific as you can, do you go about doing that? Do you include different forms of media (e.g. photos/images, audio/video, links to other media) in your tweets?

- For each of the different forms of media that you just mentioned, how do you think each individual form of media impacts the reach of your Tweet? For example, do you think that a Tweet that includes photos or audio/visual content, in addition to text, will generate more "views", "likes" or "retweets"? Why?

- Do you use the @function to tag an audience? If so, can you tell me a little bit about why you do this? And who do you tend to tag? E.g., individual accounts, broadcast media outlets, NGO's, community groups, your team?

- - Off the top of your head, can you name a few Twitter accounts that are active in the accessible public transit conversation on Twitter right now (conventional or para service)? 
- Do you use the \#hashtag function to participate in conversations about trending topics related to accessible public transit (e.g. \#ParaParadis, \#OCTranspo, \#ParaTranspo \#PWD)

- Off the top of your head, can you name a few active \#hashtags in the accessible public transit conversation on Twitter right now (conventional or para service)?

1.

2 .

3 .

4 .

5 .

- What are the main issues being raised on Twitter, as you understand them, by para transit users and their activists/lobbyists right now?

1.

2 .

3 .

4.

5 .

- When you are actively engaged in conversations at the public transit decisionmaking table, i.e., Transit Commission, how do you present your statements/motions/argumen I imagine that you prepare some notes ahead of time, do you ever reference active social media conversations at the decision-making table?

- Do you have the ability to introduce audio/video material as a knowledge generating tool, say in addition to a prepared statement/motion that is being discussed at the decision-making table?

- What is the most challenging aspect about interacting with twitter users who constantly express their dismay about the state of the public transit system via Twitter (e.g., 280-character limit, lack of clarity about an issue, several mentions about the same topic, information overload)? 
Thank you for sharing your time with me today. Before we part ways, would you like to hear a little bit about the nature of the project that we're working on? Great!

We are speaking with three groups of people for purpose this project. The three groups of people are:

1. People who self-identify as a person living with a disability who uses public transit

2. Representatives from community groups/NGOs who advocate/lobby on behalf of people who self-identify as a person living with a disability who uses public transit

3. Elected and Appointed officials who hold voting rights at the public transit decision-making table

So, what are we hoping to do with the information gathered from today's interview?

We are looking to harvest the active Twitter conversation about accessible public transit in Ottawa, with a deliberate focus on identifying the needs of the PWD community, as they are defined and discussed by the PWD community. To do this, we secured a Twitter Developer Account, which allows us to make use of the Twitter API (Application Programming Interface) to pull raw Twitter data, store it in our database, and then present this data to the aforementioned stakeholder groups in the form of an interactive atlas, that serves to empower and unite the PWD community, and better define the issues of greatest importance to the PWD community, for the public transit decision-makers. 


\section{Appendix F}

\section{Remote Usability-Testing Session}

\section{F.1 Letter of Invitation}

Title: Development From Within: An In-Service Public Transit Narrative

Date of ethics clearance: February 202020

Ethics Clearance for the Collection of Data Expires: February 282022

Dear Sir or Madam,

First, I would like to thank you for agreeing to participate in my master's thesis project in Human-Computer Interaction at Carleton University. In the semistructured interview, we briefly discussed your public transit routines, and then we discussed your use of the social media platform, Twitter, to participate in the accessible public transit conversation happening on the platform.

The second part of my study involves a virtual usability-testing session. You may recall that, prior to the covid-19 pandemic, we were able to meet in a mutually agreed upon public location to complete the semi-structured interview. At this time, we are not able to meet in a mutually agreed upon public location, and we must complete the usability-testing session virtually. In order to complete the usabilitytesting session virtually, I am asking that you attend a 45-minute virtual meeting on the Zoom web platform. On the day of the usability-testing session, I will email you a one-page document outlining the tasks that you will be asked to complete, and I will provide you with a ten-question post-test questionnaire. Please keep in mind, that the purpose of the usability-testing session is to evaluate the prototype and not the person (you). Essentially, I am asking you to use the prototype so that the research team can learn about the things that need to be done in order improve the design and functionality.

The prototype was built using specialized software on my personal computer. I am not asking you to download and install specialized software on your personal device. Instead, I am asking you to open the prototype using your preferred web browser on your native device, and enable the 'audio/video' and 'screen share' functions on the Zoom web platform. This way, I can observe you interacting with the prototype on your own device, while respecting the physical distancing guidelines mandated by the public health authority.

With your consent, the usability-testing session will be audio and video recorded (audio/video recording is a condition of participation in the study). Once the recordings have been transcribed, the audio and video recordings will be destroyed. While 
this project can involve some professional and emotional risks in the event of a data breach of the primary investigator's personal computer and external hard drive, care will be taken to protect your identity. This will be done by keeping all responses anonymous and allowing you to request that certain responses not be included in the final project. Should you feel any distress following your participation in this project, you are invited to consult mental health resources in your community-please visit www.centretownchc.org or http://www.carlington.ochc.org for additional information.

You will have the right to end your participation in the study at any time, for any reason during a session, and up to seven (7) days following your participation in the usability-testing session. If you choose to withdraw, all the information you have provided will be destroyed.

As a token of appreciation, I will be providing you with a Tim Horton's gift card valued at twenty $(\$ 20)$ dollars at the conclusion of the usability-testing session. There is no compensation if you choose not to participate in the usability-testing session.

All research data, including audio and video recordings and any digital notes will be password protected. Any hard copies of data (including any handwritten notes or USB keys) will be kept in a locked cabinet at Carleton University or in a locked cabinet in the researcher's private residence. Research data will only be accessible by the principal investigator (Ryan Grenon), the research supervisor (Dr. Fraser Taylor) and the graduate program supervisor (Dr. Robert Biddle).

The ethics protocol for this project was reviewed by the Carleton University Research Ethics Board, which provided clearance to carry out the research. Should you have questions or concerns related to your involvement in this research, please contact:

\section{CUREB-B:}

If you have any ethical concerns with the study, please contact the Carleton University Research Ethics Board-B (by phone at 613-520-2600 ext. 4085 or via email at ethics@carleton.ca). If you have any questions, please contact the principal researcher via email at ryangrenon@cmail.carleton.ca or by phone at 1+ (613) 8062217.

Sincerely,

Ryan Grenon 


\section{F.2 Informed Consent Form}

Name and Contact Information of Researchers

Name: Ryan Grenon

Email: ryangrenon@cmail.carleton.ca

Project Supervisor: Dr. Fraser Taylor

\section{Project Title}

Development From Within: An In-Service Public Transit Narrative

Carleton University Project Clearance

Clearance \# 112088

Date of Clearance: February 202020

\section{Invitation}

The information in this form is intended to help you understand what we are asking of you so that you can decide whether you agree to participate in this study or not. Your participation in this study is voluntary, and a decision not to participate will not be used against you in any way. As you read this form, and decide whether to participate, please ask all the questions you might have, take whatever time you need, and consult with others as you wish.

\section{What is the purpose of the study?}

This study aims to utilize social media data to better understand the needs of people who self-identify as a person who lives with a disability and uses public transit in Ottawa, Canada.

\section{What will I be asked to do?}

If you agree to take part in the study, we will ask you to:

- Participate in one semi-structured interview (30 minutes). The interview session will take place in a mutually convenient, safe location (e.g. a coffee shop or public library), and the session will be audio-recorded - this is a condition for participation in this study.

- Participate in one virtual usability-testing session, i.e., participate in a virtual meeting on the Zoom web platform, and complete one post-test questionnaire via email (45 minutes). The session will be audio/video recorded, and you will be asked to use the 'screen share function while using the prototype - this is a condition for participation in this study.

\section{Risks and Inconveniences}

While this project can involve some professional and emotional risks in the event of a data breach of the primary investigator's personal computer and external hard drive, 
care will be taken to protect your identity. Should you feel any distress following your participation in this project, you are invited to consult mental health resources in your community - please visit www.centretownchc.org or http://www.carlington.ochc.org for additional information.

\section{Possible Benefits}

Your participation in this study may allow researchers to better understand the needs of people who self-identify as a person who lives with a disability that limits their ability to use public transit in Ottawa, Canada. This may lead to the creation of a public database intended to serve the PWD community, the PWD advocate/lobby, and public transit decision-makers.

\section{Compensation/Incentives}

You will receive a "Thank You" card at the conclusion of the semi-structured interview, and you will receive a "Tim Horton's" gift card valued at twenty (\$20) dollars at the conclusion of the usability-testing session.

\section{No waiver of your rights}

By signing this form, you are not waiving any rights or releasing the researchers from any liability.

\section{Withdrawing from the study}

If you withdraw your consent during the course of the study, all information collected from you before your withdrawal will be discarded.

After the study, you may request that your data be removed from the study and deleted by sending an email to the Principal Investigator (ryangrenon@cmail.carleton.ca) within seven (7) days of participation in the usability-testing session.

\section{Confidentiality}

We will treat your personal information as confidential, although absolute privacy cannot be guaranteed. No information that discloses your identity will be released or published without your specific consent. Research records may be accessed by the Carleton University Research Ethics Board in order to ensure continuing ethics compliance.

The results of this study may be published or presented at an academic conference or meeting, but the data will be presented so that it will not be possible to identify any participants unless you give your expressed consent. You will be assigned a code so that your identity will not be directly associated with the data you have provided. All data, including coded information, will be kept in an encrypted file on a secure computer.

\section{Data Retention}


After the study is completed, your de-identified data will be retained for future research use.

\section{New information during the study}

In the event that any changes to the study occur you will be promptly informed.

\section{Ethics Review}

This project was reviewed and cleared by the Carleton University Research Ethics Board B. If you have any ethical concerns with the study, please contact Carleton University Research Ethics Board (by phone at 613-520-2600 [ext. 4085] or by email at ethics@carleton.ca).

\section{Statement of consent}

I voluntarily agree to participate in this study. Yes or No

I agree to be audio/video recorded/photographed Yes or No

I agree to be contacted for follow up research Yes or No

Signature of Participant:

Date:

\section{Research team member who interacted with the participant}

I have explained the study to the participant and answered any and all of their questions. The participant appeared to understand and agree. I provided a copy of the consent form to the participant for their reference.

Signature of researcher:

Date: 


\section{F.3 Usability-Testing Script}

\section{Use cases for PG1}

\section{Use case 1}

1. Have the participant view the landing page.

- "What are you looking at?"

- "What are you thinking?"

- "Is this what you expected to see?"

2. Ask the participant to explore the functionality of the landing page.

- "Do you care to explore anything on this page?" Encourage the participant to interact with the icons/buttons on the landing page.

- "What would you do if I wasn't here?" "I'd like you to do whatever you'd normally do."

- If the participant suggests concern that they are not giving the PI what they need. "No, this is very helpful." "This is exactly what we need."

\section{Use case 2}

1. Have the participant perform a database search.

- Let's pretend that you are interested in viewing all public tweets that meet the following criteria:

(a) Include the phrase "ParaTranspo"

(b) Include the hashtag "\#PWD"

(c) Tweeted on "January 1 2021"

(d) Include a media attachment "image"

- "What are you going to do first?" "What are you thinking?"

- "Is that what you expected to happen?"

- "I can't answer that right now, because we need to know what you would do when you don't have somebody around to answer questions for you. But if you still want to know when we're done, I'll be glad to answer then."

- Once the participant has finished entering their query, and they have clicked the 'search' button, the PI will ask the participant to share their thoughts about the 'search results' page. 
- "Is this what you expected to happen?"

- "What are you looking at?"

- "What are you thinking?"

- "What do you think about the results page?" "Was there something in particular that made you think that?"

- "Go ahead, explore a little bit, please select one of the icons on the map."

- "Is this what you expected to happen?"

- "What are you looking at"

- "What are you thinking"

Concluding the usability-testing session:

1. Do you have any thoughts that you'd like to share about today's session? Any feedback you provide will help the research team improve the design and functionality of any potential software build?

2. Thank you for sharing your time with me today. It is incredibly helpful to have your feedback BEFORE our team considers a software build. For one thing, it helps us better understand the potential market for a product similar to the prototype we used today.

3. We believe that the best software builds are completed hand-in-hand with our community partners from day one; this way, we don't waste your time by asking you to test a product that you didn't ask for, and our time is not wasted building a product that doesn't address your needs.

4. The last thing I will ask of you is to complete a ten-question survey that is in your inbox right now. This will help us to establish a preliminary understanding about today's virtual usability-testing session.

5. Thank you for your time. Please tell me how I can get the $\$ 20$ Tim Horton's gift card to you, while respecting your privacy and physical distancing guidelines. 


\section{Use cases for PG2}

\section{Use case 1}

1. Have the participant view the landing page.

- "What are you looking at?"

- "What are you thinking?"

- "Is this what you expected to see?"

2. Ask the participant to explore the functionality of the landing page.

- "Do you care to explore anything on this page?" Encourage the participant to interact with the icons/buttons on the landing page.

- "What would you do if I wasn't here?" "I'd like you to do whatever you'd normally do."

- If the participant suggests concern that they are not giving the PI what they need. "No, this is very helpful." "This is exactly what we need."

\section{Use case 2}

1. Have the participant perform a database search.

- Let's pretend that you are interested in viewing all public tweets that meet the following criteria:

(a) Include the phrase "ParaTranspo"

(b) Include the hashtag "\#PWD"

(c) Include the active Twitter handle "@OttTransitRider"

(d) Tweeted on "January 1 2021"

(e) Include a media attachment "image"

- "What are you going to do first?" "What are you thinking?"

- "Is that what you expected to happen?"

- "I can't answer that right now, because we need to know what you would do when you don't have somebody around to answer questions for you. But if you still want to know when we're done, I'll be glad to answer then."

- Once the participant has finished entering their query, and they have clicked the 'search' button, the PI will ask the participant to share their thoughts about the 'search results' page.

- "Is this what you expected to happen?" 
- "What are you looking at?"

- "What are you thinking?"

- "What do you think about the results page?" "Was there something in particular that made you think that?"

- "Go ahead, explore a little bit, please select one of the icons on the map."

- "Is this what you expected to happen?"

- "What are you looking at"

- "What are you thinking"

Concluding the usability-testing session:

1. Do you have any thoughts that you'd like to share about today's session? Any feedback you provide will help the research team improve the design and functionality of any potential software build?

2. Thank you for sharing your time with me today. It is incredibly helpful to have your feedback BEFORE our team considers a software build. For one thing, it helps us better understand the potential market for a product similar to the prototype we used today.

3. We believe that the best software builds are completed hand-in-hand with our community partners from day one; this way, we don't waste your time by asking you to test a product that you didn't ask for, and our time is not wasted building a product that doesn't address your needs.

4. The last thing I will ask of you is to complete a ten-question survey that is in your inbox right now. This will help us to establish a preliminary understanding about today's virtual usability-testing session.

5. Thank you for your time. Please tell me how I can get the $\$ 20$ Tim Horton's gift card to you, while respecting your privacy and physical distancing guidelines. 


\section{Use cases for PG3}

\section{Use case 1}

1. Have the participant view the landing page.

- "What are you looking at?"

- "What are you thinking?"

- "Is this what you expected to see?"

2. Ask the participant to explore the functionality of the landing page.

- "Do you care to explore anything on this page?" Encourage the participant to interact with the icons/buttons on the landing page.

- "What would you do if I wasn't here?" "I'd like you to do whatever you'd normally do."

- If the participant suggests concern that they are not giving the PI what they need. "No, this is very helpful." "This is exactly what we need."

\section{Use case 2}

1. Have the participant view the dashboard page.

- "What are you looking at?"

- "What are you thinking?"

- "Is this what you expected to see?"

2. Ask the participant to explore the functionality of the dashboard page.

- "Please select a tweet story, curated by one of our community partners, to explore in greater detail"

- "What are you looking at?"

- "What are you thinking?"

- "Is this what you expected to see?"

- "Is this information useful to a public transit decision-maker such as yourself"

- "What is missing? "What can we do to improve this page to the point where it may become a useful tool in your toolbox for public transit decision-making?"

Concluding the usability-testing session: 
1. Do you have any thoughts that you'd like to share about today's session? Any feedback you provide will help the research team improve the design and functionality of any potential software build?

2. Thank you for sharing your time with me today. It is incredibly helpful to have your feedback BEFORE our team considers a software build. For one thing, it helps us better understand the potential market for a product similar to the prototype we used today.

3. We believe that the best software builds are completed hand-in-hand with our community partners from day one; this way, we don't waste your time by asking you to test a product that you didn't ask for, and our time is not wasted building a product that doesn't address your needs.

4. The last thing I will ask of you is to complete a ten-question survey that is in your inbox right now. This will help us to establish a preliminary understanding about today's virtual usability-testing session.

5. Thank you for your time. Please tell me how I can get the $\$ 20$ Tim Horton's gift card to you, while respecting your privacy and physical distancing guidelines. 


\section{Appendix G}

\section{Bus Stop Accessibility - A Mathematical Process}

In an extension of the in-text example (i.e., a judge measuring the accessibility of a bus stop), we introduce the following mathematical process.

\section{Should the Transit Commission reduce noise in decision-making activities?}

Suppose the database query and results visualization tool introduced in this thesis is adopted by the Transit Commission. And suppose it is consulted at regular intervals when public transit decision-makers must weigh the pros and cons of various policymaking options. If we acknowledge that no evaluation of policymaking options is possible without accurate predictions about the likely consequences of each option (including the option to do nothing, i.e., maintain the status quo), should the Transit Commission use the database query and results visualization tool to inform the design of risk policies that combine the experts' knowledge with the public's emotions and intuitions? We show how the application of various rubrics/lenses (e.g., accessibility, equity, etc.) can be used to increase the social and economic value of the public transit system in Ottawa through more precise decision-making activities, undertaken by each of the three public transit stakeholder groups we engaged with.

The database query and results visualization tool is designed to collect and organize multiple independent measurements on the same item, and present these measurements in interactive information visualizations for decision-makers (i.e., public transit stakeholders), using location as the central organizing principle. When multiple measurements on the same item are considered together (i.e., averaged), noise (i.e., variability in the measurements) will diminish because errors cancel out.

The Transit Commission (i.e, each individual Commissioner) has reviewed the social media data as they typically do (i.e., the existing IS), and produced an independent estimate of bus stop accessibility for one neighbourhood (i.e., thirteen independent measurements using thirteen independent rubrics).

Figure G.1 shows the (implausibly smooth) results of the accessibility forecasts. You can see that the forecasts are distributed in the familiar Gaussian distribution (i.e., bell shaped curve). The most frequent forecast, represented by the peak of the bell curve, is $44 \%$. You can also see that the forecasts are quite noisy - the forecasts, which would be identical if each Commissioner used the same underlying dataset, vary over a considerable range. 


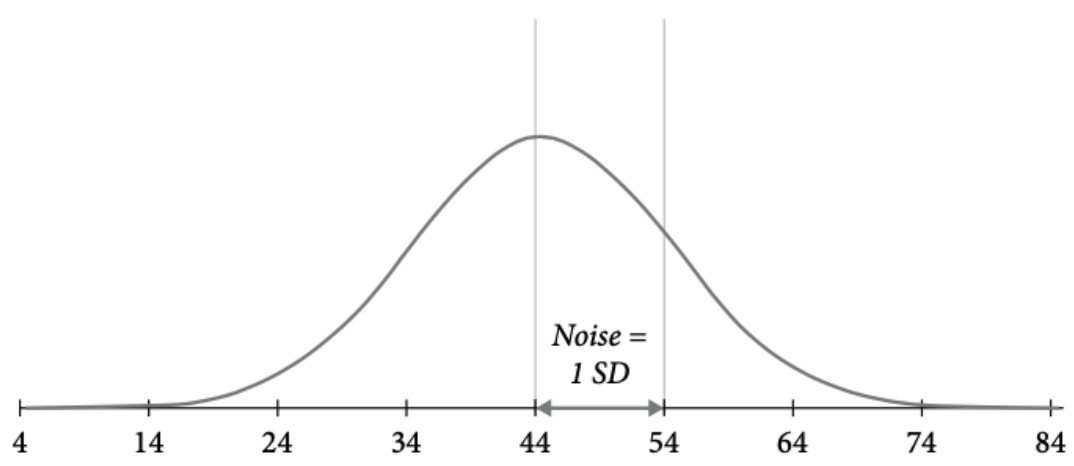

Figure G.1: Distribution of the Transit Commission's Bus Stop Accessibility Forecasts for One Neighbourhood

We can attach a number to the amount of noise in the forecasting system - we can compute the standard deviation of the independent forecasts. In this example, the standard deviation is 10 percentage points. As is true for every normal distribution, about two-thirds of the forecasts are contained within one standard deviation on either side of the mean - in this example, between $34 \%$ and $54 \%$ of the bus stops were forecasted to be accessible. We now have an estimate of the amount of system noise in the accessibility forecasts. (A better noise audit would use several forecasts of several items for a more robust estimate.)

One member of the Transit Commission is shocked by the difference in forecasts and wants to take action. The unacceptable amount of noise indicates that the Transit Commission, as a whole, is not disciplined in implementing the procedures they are expected to follow - the Transit Commissioners continue to use single viral social media posts to inform their perceptions of bus stop accessibility. The Commissioner asks the Chair of the Transit Commission to consider using the database query and results visualization tool discussed in this thesis to achieve more uniformity and discipline in the forecasting process (i.e., the Commissioner asks if all Commissioners should produce independent forecasts using the same underlying dataset). The Chair denies the Commissioner's request to use the database query and results visualization tool, but asks "how can we reduce errors when we don't know if the forecasts are right or wrong? Surely, if there is a large average error in the forecasts (i.e., large bias), addressing it should be the priority. Before undertaking any measures to improve the forecasts, the Transit Commission must wait and find out if the forecasts are in fact correct."

One year after the initial forecasts, the outcome that the Transit Commissioners were trying to predict is known. Accessibility of the bus stops in the neighbourhood turned out to be $34 \%$. Now we also know each Commissioner's error, which is simply the difference between the Commissioner's forecast and the true outcome. The error is 0 for a forecast of $34 \%$, it is $10 \%$ for the mean forecast of $44 \%$, and it is $-10 \%$ for a low-ball forecast of $24 \%$. 
Figure G.2 shows the distribution of errors in the set of forecasts. It is the same as the distribution of forecasts in Figure G.1, but the true value, (34\%), has been subtracted from each forecast. The shape of the distribution has not changed, and the standard deviation (i.e., noise) is still $10 \%$.

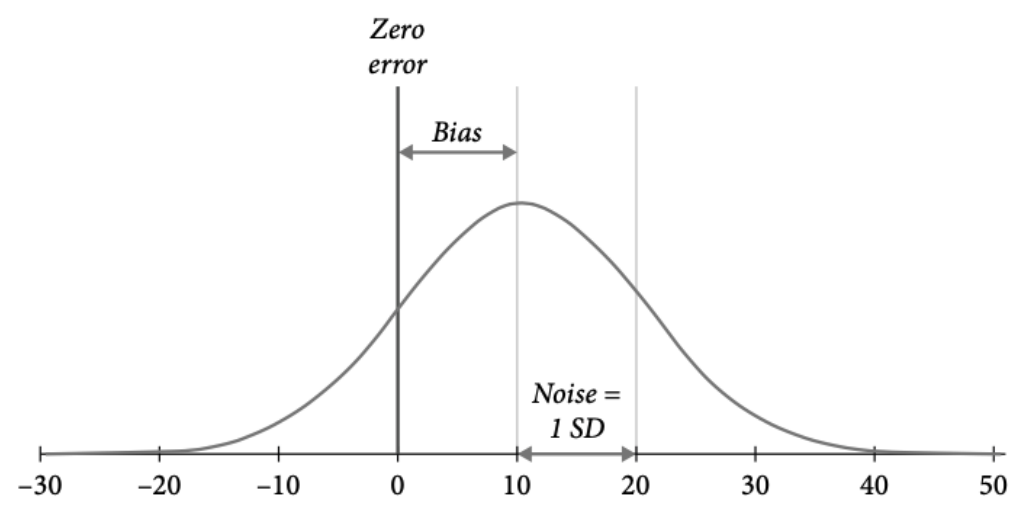

Figure G.2: Distribution of Errors in the Transit Commission's Bus Stop Accessibility Forecasts for One Neighbourhood

The difference between between Figure G.1 and Figure G.2 is analogous to the difference between a pattern of shots seen from the back and the front of the target in Figure G.3. Knowing the position of the target was not necessary to observe noise in shooting; similarly, knowing the true outcome adds nothing at all to what was already known about noise in forecasting.

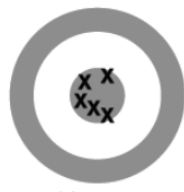

TeAM A

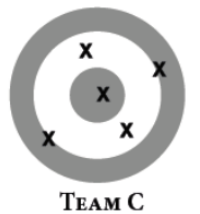

(a) Looking at the Front of the Target

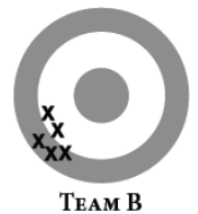

TеАм D

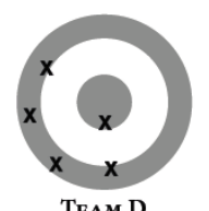

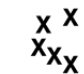

TEAM A

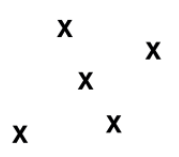

TеАм C

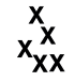

TEAM B
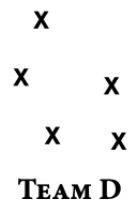

(b) Looking at the Back of the Target

Figure G.3: Shots Fired

The Transit Commissioner who wanted to take action, and the Chair who wanted to wait for the true outcome to be known before taking any action, now know something they did not know earlier: the amount of bias in the Commissioners' forecasts. 
Bias is simply the average of errors, which in this case is also $10 \%$. Bias and noise, therefore, happen to be numerically identical in this set of data. (To be clear, this equality of noise and bias is by no means a general rule, but a case in which bias and noise are equal helps to understand their roles.) We can see that most forecasters made an optimistic error - that is, they overestimated the level of bus stop accessibility that would be achieved: most of the Transit Commissioners erred on the right-hand side of the zero-error vertical bar. (In fact, using the properties of the normal distribution, we know that is the case for $84 \%$ of forecasts.)

As the Chair notes with hardly concealed satisfaction, they were right. There was a lot of bias in the forecasts. And indeed, it is now evident that reducing bias would be a good thing. But, the concerned Transit Commissioner still wonders, would it have been a good idea a year ago - and would it be a good idea now - to reduce noise? How would the value of such an improvement compare with the value of reducing bias?

To answer the Transit Commissioner's question, we need a "scoring rule" for errors, a way to weight and combine individual errors into a single measurement. The method of least squares, introduced in chapter 5, is the appropriate measure because it builds on an intuition that most people share. When trying to estimate a true outcome from a set of measurements, one possibility is to select the median number - the measurement that sits in the middle of the set - the other possibility is the arithmetic mean (i.e., the average). Your intuition probably favours the mean, and your intuition is correct. The mean contains more information; it is affected by the size of the numbers, while the median is affected only by their order. The best estimate is one that minimizes the overall error of the available measurements; therefore, the formula the Transit Commission should use to measure overall error should be the one that yields the arithmetic mean as the value for which error is minimized.

The error equation provides unequivocal support for the Transit Commissioner's initial impulse to try to reduce noise. The equation shows that the Chair was wrong when they suggested that the Transit Commission wait to measure the bias in its forecasts, and only then decide what to do. In terms of overall error, noise and bias are independent: the benefit of reducing noise is the same, regardless of the amount of bias.

This notion is highly counter-intuitive but critical. To illustrate it, Figure G.4 show the effect of reducing bias and noise by the same amount. The original distribution of errors ( Figure G.2) is represented with a broken line. 
Panel A: Same Noise, Less Bias

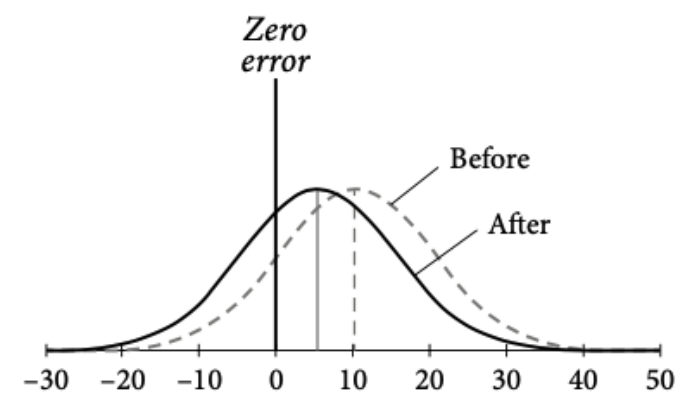

Panel B: Same Bias, Less Noise

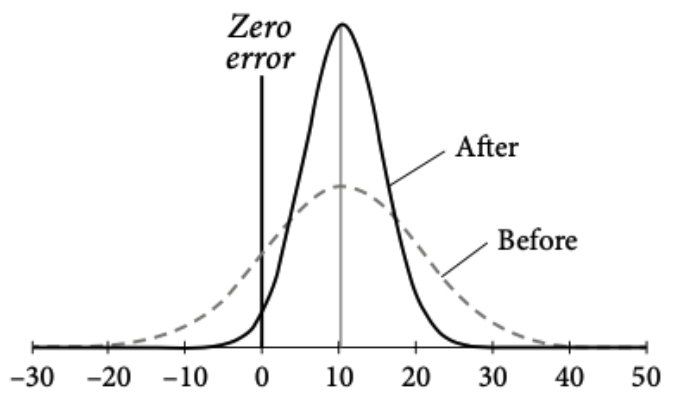

Figure G.4: Distribution of Errors with Bias Reduced by Half vs. Noise Reduced by Half

In Panel A, we assume that the Chair decided to do things their way: they found out what the bias was, then somehow managed to reduce it by half (perhaps by providing feedback to the overoptimistic Commissioners). Nothing was done about noise. The improvement is visible: the whole distribution of forecasts has shifted closer to the true value.

In Panel B, we show what would have happened if the Transit Commissioner had convinced the Chair of her argument. Bias is unchanged, but noise is reduced by half. The paradox here, is that a reduction of noise seems to have made things worse. The forecasts are now more concentrated (i.e., less noisy), but not more accurate (not less biased). Whereas $84 \%$ of forecasts were on one side of the true value, almost all $(98 \%)$ now err in the direction of overshooting the true value. Noise reduction seems to have made the forecasts more precisely wrong - not the improvement the Transit Commissioner had hoped.

Despite appearances, however, overall error has been reduced just as much in Panel $\mathrm{B}$ as in Panel A. The illusion of deterioration in Panel B arises from an erroneous intuition of bias. The relevant measure of bias is not the imbalance of positive and negative errors. It is average error, which is the distance between the peak of the bell curve and the true value. In Panel B, the average error has not changed from the original forecasts - it is still high, at 10\%, but not worse. True, the presence of bias is now more striking, because it accounts for a larger proportion of overall error $(80 \%$ rather than 50\%). But that is because noise has been reduced. Conversely, in Panel A, bias has been reduced, but noise has not. The net result is that MSE is the same in both panels; reducing noise or reducing bias by the same amount has the same effect on MSE.

As this example illustrates, MSE conflicts with common intuitions about the scoring of predictive judgments. To minimize MSE, you must concentrate on avoiding large errors. If you measure length, for example, the effect of reducing an error from $11 \mathrm{~cm}$ to $10 \mathrm{~cm}$ is 21 times as large as the effect of going from an error of $1 \mathrm{~cm}$ to a perfect hit. Unfortunately, people's intuitions in this regard are almost always the mirror 
image of what they should be: people are very keen to get perfect hits and highly sensitive to small errors, but they hardly care at all about the difference between two large errors.

Of course, the best solution here is to reduce both noise and bias. Since bias and noise are independent, there is no reason to choose not to reduce noise (unless there is something to hide, of course). If the Transit Commission decides to reduce noise, the fact that noise reduction makes bias more visible - indeed, impossible to miss may turn out to be a blessing.

Admittedly, reducing noise would be less of a priority if bias were much larger than noise. But the Transit Commission example offers another lesson worth highlighting. In this simplified model, we have assumed that noise and bias are equal. Given the form of the error equation, their contributions to total error are equal too: bias accounts for $50 \%$ of overall error, and so does noise. Yet, as we have noted, $84 \%$ of the Commissioner's err in the same direction. It takes a bias this large (six out of seven people making mistakes in the same direction) to have as much effect as noise has on overall error. We would not be surprised, therefore, to find situations in which there is more noise than bias.

We illustrated the application of the error equation to a single case: bus stop accessibility for a set of bus stops in one particular Ottawa neighbourhood. Of course, it is always desirable to evaluate noise on multiple cases at once. To do this, the error equation should applied to multiple sets of bus stops in multiple neighbourhoods; and an overall equation is obtained by taking the averages of MSE, bias squared and noise squared over the sets of bus stops. It would have been better for the Transit Commission to obtain multiple forecasts for multiple sets of bus stops, in several neighbourhoods, either from the same or from different Commissioners. Averaging results would give a more accurate picture of bias and noise in the Transit Commission's overall forecasting system.

The Transit Commission Forecasting example (including the figures) is an adaptation of the 'Goodsell market forecasting' example, originally presented in Noise: A Flaw in Human Judgment by Daniel Kahneman, Olivier Sibony, and Cass R. Sunstein [58]. 